\title{
WILEY-VCH
}

\section{Track-etched Nanopore/Membrane: From Fundamental to Applications}

Tianji Ma, Jean-Marc Janot, Sébastien Balme*

Dr. T. Ma, Dr. J-M Janot, Dr S. Balme

Institut Européen des Membranes, UMR5635 UM ENCSM CNRS, Place Eugène Bataillon, 34095 Montpellier cedex 5, France

E-mail : sebastien.balme@umontpellier.fr

Keywords: track-etched membrane, nanopore, nanofluidic, biomimetic

The biomimetic and bio-inspired membranes have drawn great attention and generated extensive efforts to solve environmental, health and energy issues. In this field, the tracketched membranes present the advantage to be tuned from a single pore to large-scale multipore membrane. On one hand, from the large-scale membrane to single nanopore, the research becomes more fundamental, meticulous and quantitative to describe concrete processes inside confined space. This allows understanding the role of the surface phenomena and nanoscale effects. On the other hand, working from a single pore to multipore membrane allow perfect control of the membrane properties. This ensures a promising ability for upscale from the lab findings to applications. In this review, we take a global insight on the tracketched nanopore/membrane through presenting fabrication and functionalization methods, transport properties and the related applications. By functionalization, track-etched nanopore can be used to understand ion transport under confined space with high aspect ratio, to analyze single molecules, to design stimuli-responsive membranes and to generate energy from salinity gradient and light.

\section{Introduction}

From single-cell organisms to human beings, biological ion channels in cell membranes play vital roles in transporting matters between internal and external environment of cells [1]. These processes on every moment regulate energy production, conversion, storage and signal propagation, conversion and processing. Currents mediated by ion channels were firstly 


\section{WILEY-VCH}

studied by Alan Hodgkin and Andrew Huxley who identified the action potential in 1950s[2]. Later in 70s, Bernard Katz and Ricardo Miledi confirmed the existence of ion channels from noise analysis[3]. Then "patch-clamp" technique was developed by Erwin Neher and Bert Sakmann as electrical recording method[4]. It became the main tool to study ion channels. More recently physicochemical properties of ion channel structure and function have been more and more revealed[5] and a third Nobel Prize related to ion channels was appointed to Roderick MacKinnon in 2003[6]. These channels not only play an important role in life and medical science but also inspire development of membrane science thank to their selectivity, rectification and gating properties[7, 8, 9, 10].

From the end of last century, nanopore technology progresses rapidly especially for single molecule sensing. The first DNA detection by nanopore was accomplished with a biological nanopore, $\alpha$-hemolysin in 1996 by Kasianowicz and co-workers[11]. From then numerous biological pores of toxins and outer-membrane channels have been exploited including aerolysin[12], MspA[13], OmpG[14], FraC[15], ClyA[16], CsgG [17], Phi-29 [18] gramicidin $\mathrm{A}[19]$, anthrax toxin[20], lysenin[21] and virus portal protein[22]. The first main achievement of biological nanopore technology was the real-time DNA sequencing[23]. It becomes more and more ripe that can respond sudden demand for Sars-cov-2 characterization rapidly which is a global urgent sanitation crisis[24]. Beside DNA, the protein sequencing is the new main challenge in this area[25].

Despite the advantages of biological nanopores such as fine microstructure and possibility to be chemically engineered in molecule sensing and DNA sequencing, their limits are also clear[26]. The size of a biological pore is usually fixed which limits one pore facing different demands. The lipid bilayer as the support can be unstable when external conditions change. Furthermore, even the biological pore can be modified by chemically engineering techniques like mutagenesis; it is still difficult to design responding to particular applications. This 


\section{WILEY-VCH}

promotes to develop a stable, size tunable, easy-functionalizable alternative known as solidstate nanopore.

Thanks to the advances in nanotechnology especially in nanofabrication, artificial solid-state nanopores have been achieved by different ways. They include (i) silicon nitride nanopores drilled by ion beam sculpting[27], TEM technique[28] or controlled dielectric breakdown (CDB) technique[29]; (ii) nanotubes (NT) synthesized by catalytic particles coupled with chemical vapor deposition[30], carbon-arc discharge and laser[31] (iii) 2D nanopores such as graphene[32], boron nitride[33] and molybdenum disulfide[34] opened by ablation via focused electron-beam irradiation in a TEM[35] (iv) nanopipette made through laser-assisted pulling[36] (v) polymer nanopore opened by track-etching technique[37]. The properties of these nanopores can be very different according to their size, geometry or surface state. The pore diameter can be sub nanometers to even micrometers and the thickness can be from one atom ( $0.3 \mathrm{~nm}$ for graphene) to micrometer scale for track-etched nanopore.

Among all artificial nanopores, track-etched nanopore can be considered as new but also old technology. It is old because track-etched membranes have been used in industry for more than a half century[38]. Then with the improvement in high energy ion beams of accelerators, the single ion track makes it possible to fabricate single nanopores in polymer films[39]. Various chemical etching processes provide tunable pore geometries like cone[40, 41], cylinder[42, 43], funnel[44], bullet[45], hourglass[46], dumbbell[47] etc. (Figure 1). These geometries offer platforms to study ions, macromolecules and nanoparticles transport phenomena in confined space[48-50]. These investigations were combined with numerical simulations based on continuum models $[51,52]$ or molecular dynamics[ $[53,54]$ to have both experimental evidence and theoretical descriptions. The second advantage of track-etched nanopore is that the surface can be easily functionalized thanks to carboxylic moieties after chemical etching process of polyethylene terephthalate or polycarbonate films. The third strong point is the possibility of upscale from single nanopore to $\mathrm{m}^{2}$ scale multipore 


\section{WILEY-VCH}

membranes. The single track-etched nanopore allows as a good proof of concept for the understanding stimuli responsiveness and ion pumping properties. Then the findings in single nanopore can be quickly upscale to stimuli-responsive and/or ion-selective membranes for applications.

With development in material science, organic chemistry, molecular biology and biophysics, track-etched nanopores show promising abilities in stimuli-responsive ion channels, ions and molecules sensing as well as energy conversion [55]. Here, we report and discuss the updated advancement in track-etched nanopores in last two decades. This review contains three sections. The first section deals with the fabrication and functionalization methods. The second section describes fundamentals in transport phenomena near the interface, in nanofluidic with or without surface charges. Finally, the last section summarized the applications of track-etched nanopores/membranes in stimuli-responsive membranes, ions gating/sensing, molecules sensing and energy conversion.

\section{Track-etched nanopore/membrane fabrication and functionalization}

\subsection{Fabrication of track-etched nanopore}

Track-etched membranes made of polymer foils were first fabricated as multipore membranes. The process follows two steps: ion tracking and chemical etching. There are two methods for producing tracks on polymer films. The first one is using fragments of nuclear fission[56]. It has low cost and stable particle flux but disadvantages like radioactive contamination, limited energy range and fragments complexity reduce the use of this method. The second method is based on high energy ion beams from accelerators[57]. Despite the high cost and lower particle flux stability, accelerator-based ion irradiation becomes more and more common due to its controllability in energy, impact angle especially for single track fabrication. For now, the single-track fabrications are possible at GSI (Darmstadt, Germany) and GANIL (Caen, France) facilities. 


\section{WILEY-VCH}

The irradiation step provides a latent track. When swift heavy ions bombard polymer films, two kinds of energy losses will happen including nuclear energy loss and electronic energy loss. Both nuclear and electronic energy loss depends on the specific energy of ions. For typical heavy ions at high velocity (high specific energy), nuclear energy loss can be neglected as long as ions completely pass through the polymer films. In this case, electronic energy loss dominates and occurs shortly before the particle is stopped, because velocity decrease increases their interaction cross section. This energy loss can be expressed by [58]:

$\frac{d E}{d x}=\frac{4 \pi e^{4} Z_{e f f}^{2} Z_{t} N}{m_{e} v^{2}}\left[\ln \left(\frac{2 m_{e} v^{2}}{I}\right)-\beta^{2}-\delta-U\right]$

where $e$ is elementary charge, $Z_{\text {eff }}$ the effective charge of the projectile, $Z_{t}$ the atomic number, $N$ the number of target atoms per unit volume, $m_{e}$ the electron mass, $v$ the ion velocity, $I$ the ionization energy, $\beta$ the relative correction and $U$ the inner electrons screening correction.

The effective penetrating range of an ion with initial kinetic energy $E_{0}$ can be calculated by integration of eq. 1 .

$\mathrm{R}=\int_{0}^{E_{0}}\left(\frac{d E}{d x}\right)^{-1} d E$

Two models have been proposed to explain the ion track formation[59]. Coulomb explosion model describes an ionization of atoms along the ion path. Electrons of atoms in track cores are ejected outside the core in a zone called "track halo". Then the positively charged area forms defects and gaps by Coulomb repulsion. The other model is called thermal spike model. It considers from a thermal point by conceiving a rapid increase of the temperature. This high temperature releases a hot electron gas to neighbor atoms and melts a cylinder track core area. After cooling down, defects, and gaps are created. Different from the track core full of defects and gaps, the track halo can be different depending polymers. For example, polycarbonate can be decomposed in the halo whereas PET undergoes cross-linking[60]. 


\section{WILEY-VCH}

Track structures have been studied by means of high-effective liquid chromatography and atomic-force microscopy[61] as well as the etching rate[62, 63]. Using the latter method, Apel et al. found that the track core size in PET film depends on ions and specific energy ranging from $7.5 \mathrm{~nm}$ to $25 \mathrm{~nm}[62]$. For track halo size, a ring thickness between $7 \mathrm{~nm}$ to $15 \mathrm{~nm}$ was measured according to ion types and specific energy[62]. Déjardin et al. measured streaming potential across PET membranes to find the variation of the polymer structure with increasing pore radius. A constant interfacial potential of $-47.3 \mathrm{mV}$ is measured in the crosslinked area with a diameter about $17 \mathrm{~nm}$. This interfacial potential of $36.2 \mathrm{mv}$ is different from that out of $70 \mathrm{~nm}$ indicating a higher charge density in the polymer gel in the halo.

The ion tracks can be reactivated under Ultra Violet light (UV) even several years after irradiation. This allows the storage of ion tracks for years with more or less changes. Typically, the UV irradiation increases the chemical activity of the tracks and thus enhances the chemical etching velocity.

The second step is chemical etching which will open the pores and determine its geometry and size (Figure 1). The etching solution attacks both track zone and polymer bulk with two different velocities $V_{t}$ and $V_{b}$ respectively. The ratio of these two rates can determine pore geometries. They depend on the polymer etchant composition, concentration, the temperature and the use of additive such as surfactant. Generally, for a high concentration etchant at room temperature, $V_{\mathrm{t}}$ is higher than $V_{b}$ but in the same range. Such conditions are suitable to obtain a conical shape pore under dissymmetrical condition. At higher temperature and lower etchant concentration, $V_{t} \gg V_{b}$ which will result in a cylindrical geometry. By combining symmetric etching (etching from both sides), asymmetric etching (etching from one side) and under condition where $V_{t} \approx V_{b}$, or $V_{t}>>V_{b}$, various shapes can be obtained including hourglass, funnel, dumbbell.

The $V_{b}$ is generally well reproducible for each material at constant chemical etching condition (temperature and concentration of etchant entities). So, the cylindrical pore diameter and the 


\section{WILEY-VCH}

large side of conical pore diameter are controlled by etching time. This requires a measurement of the $V_{b}$ in advance. The $V_{t}$ is affected by the trajectory of ions and the damages are more affected by the local structure of the polymer. The control of the tip diameter is difficult for the conical nanopore. In this case, the etching process is usually combined with a current measurement under constant voltage devices. Using this, the nanopore opening is monitored and the tip diameter can be estimated from the current based on which the etching time can be determined.

The use of surfactants allows forming cigar-like and bullet-like shapes[64] (Figure 1b). Surfactant molecules are adsorbed to entrance surface to protect it from etching. Then the etchant solution will attack inner space with a small opening. The latter depends on the length of surfactant molecules that defines the length of protected zone. It should be noticed that it exists a minimum pore diameter of track-etched nanopore because of the track size and the etching properties. A minimum of $2 \mathrm{~nm}$ was achieved for conical pores, while for cylindrical pores, the limit is upper than $20 \mathrm{~nm}$.

Several polymers have been used to design track-etched membrane including polyethylene terephthalate (PET)[37], polycarbonate (PC)[60], polyimide (PI)[65], polyvinylidene fluoride (PVDF)[66] and polypropylene (PP)[67]. The PET is one of the most studied materials for track etching technology. It is relatively stable in acid and organic solvent with good mechanical property and insulators. The classical method for etching is using alkaline solutions such as $\mathrm{KOH}$ or $\mathrm{NaOH}$. For conical single nanopore, PET films are the most used. After chemical etching, the PET pore surface has carboxylate moieties which are ideal for chemical functionalization by forming amides, ester bonds as well as for polyelectrolyte adsorption by electrostatic interactions. The PC is the second most used polymer for tracketched membrane. PC membrane has similar properties of PET with higher sensitivity and lower resistance to organic solvents and a lower wettability. The chemical etching is also performed under similar condition than PET. PC is more suitable to produce membrane with 


\section{WILEY-VCH}

cylindrical nanopore because the pore size is very homogeneous and smooth. This is the reason why PC is used for commercial membrane for liposome production by extrusion technique[68]. PI membrane differs from PET and PC by its excellent stability at high temperatures. $\mathrm{NaClO}$ and $\mathrm{H}_{2} \mathrm{O}_{2}$ can be a good etchant solution for PI[64] while instability of the etchants in time at high temperatures limits its use[69]. PVDF is highly hydrophobic material. Its chemical etching requires using permanganate solution in a concentrated alkaline medium[70]. PP as a hydrophobic material can be fabricated as membrane for strong alkali solutions or inorganic acids filtration due to high chemical stability[71]. Wherefore, it can be difficult to be etched with this high stability. Strong oxidizers like chromium trioxide can be used. Other materials were considered for the track-etched nanopore designs to form heterogeneous membranes such as mica[72], graphene[73] or graphene oxide[74]. The track-etched membrane can be done on polymer film thickness from $6 \mu \mathrm{m}$ to $50 \mu \mathrm{m}$ for PC, PET, PVDF and PI. A thick film provides better mechanical properties for the membrane specially after a chemical etching of a long time. However, it can also decrease the ion selectivity in membrane uses and sensitivity in single pore sensing. The thickness is also depending is also governed by the energy of swift heavy ions and the polymer used [63]. So the most commonly used track-etched nanopore/membranes have thickness from a couple to teens $\mu \mathrm{m}$. For single nanopore experiments, $6 \mu \mathrm{m}$ thickness provides a higher rectification behavior.

\subsection{Functionalization}

Track-etched membranes require functionalization prior to the applications. The introduction of new properties into the existing membranes by surface modification is compulsory in developing new membranes but also single nanopore/nanochannels. With good stability, surface and electric property, there are abundant functionalization methods to change surface property and the pore size. These methods can (i) provide stimuli responsiveness for smart membranes (ii) import bioactive molecules for sensors or (iii) enhance ion selectivity for 


\section{WILEY-VCH}

energy harvesting. To achieve that, it is important to select surface modification methods suitable to functionalize the inner wall of nanopores under confined space.

\subsubsection{Deposition techniques}

Here, we present three deposition techniques (i) physical and chemical vapor deposition (ii) atomic layer deposition and (iii) electroless deposition. These methods can be essentially used to introduce inorganic layers inside the pore or on the membrane surface at the same time. These modifications are useful to change pore surface properties and pore size. They also give abilities for further chemical functionalization.

\section{Physical and chemical vapor deposition}

These two techniques are typical asymmetric deposition using vapor as resources. They differ on two depending whether a chemical reaction or a physisorption is involved. For physical vapor deposition (PVD), the resource vapor can be generated by ion sputtering or electron beam evaporation. They use the same principle but different beams of ions or electrons to bombard target solid to eject atoms as vapor in the reactor. The ejected atoms will be deposited on the substrate to form a thin film. Track-etched nanopore can be functionalized by PVD to deposit metal films such as $\mathrm{Au}$ or $\mathrm{Pt}$ and non-metal films such as $\mathrm{SiO}_{2}$. This allows depositing a dense and uniform film with high adhesion. However, the vapor deposition can deliver resource atoms from only one direction which limits the deposition inside the nanopore for a long depth. Thus for a uniform deposition along the pore is impossible. $\mathrm{Ar}^{+}$is a common used ion resource for ion sputtering. Using this, Jiang's group has designed biomimetic asymmetric ion channels by depositing metal films in conical[75, 75] and hourglass[76] nanochannels. In a conical nanopore, they covered the whole inner wall by a layer of Pt[75]. Because of its asymmetric deposition property, it is almost impossible to cover the whole pore surface by a single deposition especially from tip side. So a comparison of covering firstly the base side then the tip side and the contrary order were made. The results show that the ion sputtering on tip side gives a significant ion rectification behavior. 


\section{WILEY-VCH}

The Pt deposition increases a lot the asymmetry of the channel. With the same method, they also designed an hourglass shape asymmetric ion channel by depositing Au and $\mathrm{Pt}$ in each cone of the channel[76]. The ion current rectification was well enhanced by introducing a different metal distribution.

Other than ion sputtering, Siwy's group deposited on the side of the membrane with the small opening a multilayer $\mathrm{Ti}-\mathrm{Au}-\mathrm{Ti}-\mathrm{SiO}_{2}$ using electron beam evaporator to construct voltage gated ionic transistor[77]. The Au electrode can change ion current rectification of the channel by varying applied electrical bias offering a new method to design ionic logic devices and ionic amplifiers.

Chemical vapor deposition (CVD) involves chemical reactions using gaseous reactance. Another difference between CVD and PVD is that it can form a more conformal deposition inside the pore $[78,79]$. Asatekin et al. used initiated chemical vapor deposition (iCVD) to functionalize track-etched membranes to tune surface hydrophobicity for separation applications[78]. This method can directly translates free radical polymerization into a chemical vapor deposition (CVD) process[80]. A poly(perfluorodecyl acrylate) (pPFDA) layer and a poly(divinylbenzene) (pDVB)- pPFDA bilayer have been deposited inside the pore. The pPFDA provides pore surface hydrophobicity and pDVB plays a role as narrower. With this functionalization, the new membrane can be used to separate molecules of similar size but with different polarity. Other than iCVD, more and more researchers turn interests in plasma enhanced chemical vapor deposition (PECVD) which allows low temperature deposition. Kelkar et al. used such method to modify pore size of track-etched membranes[79]. A comparative study between PVD, PECVD and atomic layer deposition (ALD) show that Pulsed PECVD produced asymmetric pores characteristic of PVD while displaying the precision and reproducibility of ALD. 


\section{WILEY-VCH}

Atomic layer deposition

The atomic layer deposition (ALD) is a subclass of CVD. It is based on sequential use of a gas phase chemical process to deposit thin films of high quality and excellent conformality[81]. The ALD usually consists of an alternate exposure of two precursors. The self-limiting property makes it possible to fabricate films without contaminants. The thin film was found homogeneous even if the pore has a high-aspect ratio[82]. The atomic layer deposition allows an extremely fine control of the film thickness in angstrom scale[83]. Various materials have been coated in track-etched nanopore by ALD as functionalization including $\mathrm{Al}_{2} \mathrm{O}_{3[49]}$ and $\mathrm{Al}_{2} \mathrm{O}_{3} / \mathrm{ZnO}[54,84,85], \mathrm{HfO}_{2[86]}$ on PET and $\mathrm{SiO}_{2[87,88]}$, $\mathrm{TiO}_{2[88]}$ and $\mathrm{Al}_{2} \mathrm{O}_{3[88]}$ on PC. Whatever the material, the deposition process has to be done at low temperature (below $100^{\circ} \mathrm{C}$ ) to keep the membrane structure. This limit the type of material that can be deposited but also their phase. Our group used $\mathrm{ALD}$ of $\mathrm{Al}_{2} \mathrm{O}_{3} / \mathrm{ZnO}$ nanolaminate deposition for diameter reduction in order to insert biological pores inside track-etched nanopores[54, 84]. Cylindrical track-etched nanopores have a minimum diameter of around $20 \mathrm{~nm}$ to $30 \mathrm{~nm}$ limited by the fabrication condition. Deposition of $\mathrm{Al}_{2} \mathrm{O}_{3} / \mathrm{ZnO}$ layer can reduce diameter from $36 \mathrm{~nm}$ to sub-10 $\mathrm{nm}$ for Gramicidin A insertion[54] and diameter from $75 \mathrm{~nm}$ to $5.4 \mathrm{~nm}$ for $\alpha$-hemolysin insertion[84]. This enables fabricating biological-synthetic hybrid nanopores for ionic transport study and biomolecule detection. $\mathrm{Al}_{2} \mathrm{O}_{3}$ layer was deposited inside conical track-etched nanopore to reduce pore diameter and to improve surface charge property for DNA detection. It was clearly shown that with $\mathrm{Al}_{2} \mathrm{O}_{3}$ polymer nanopore surface, the signal noise was largely decreased in resistive pulse providing a more precise analyzing. Beside of transient sensing, Lepoitevin et al. coated cylindrical nanopore by $\mathrm{Al}_{2} \mathrm{O}_{3} / \mathrm{ZnO}$ for further biotinylation which permits bio-conjugate molecules grafting for steady state biosensing[85]. 


\section{WILEY-VCH}

\section{Electroless plating}

The electroless plating is film deposition method in aqueous solutions at room temperature. It uses autocatalytic redox reaction to reduce cations into metallic layers on the substrate. Firstly, the substrate surface will be pretreated by introducing a catalyzer and then the pretreated surface will be immersed in a plating solution containing cations for ion reduction. This method is quite suitable for track-etched polymer membrane because it runs in solution and the temperature for electroless plating is relatively low[89]. Metallic layers deposited by this method are usually dense and their thickness can be well controlled. A disadvantage of it involves the frequent use of $\mathrm{Sn}$ ions which can be toxic[90].

Numerous metals can be deposited by electroless plating including $\mathrm{Au}, \mathrm{Ni}, \mathrm{Ag}$ and $\mathrm{Cu}$ among which the most used for track-etched nanopore is $\mathrm{Au}[91]$. The latter enables the surface for further self-assembled monolayers (SAMs) cover relying on thiol-Au coordination[92]. Chun et al. used this method to cover PC membrane by Au coordinated 11-Mercaptoundecanoic acid ( $\left.\mathrm{HS}\left(\mathrm{CH}_{2}\right)_{10} \mathrm{COOH}\right)$ SAMs which permit studying protein transport[93]. Asandei et al. constructed a pH-switchable membrane by using Au-plated, L-cysteine chemisorbed PC membrane[94]. The Au electroless deposition was also used to fabricate replicate of conical nanopore in order to characterize well the shape by SEM or TEM[95].

\subsubsection{Organic molecules}

Organic molecules including small organic molecules and polymers have been largely used for track-etched membrane functionalization[96, 97]. Small organic molecules can introduce specific structures for ion sensing $[98,99]$, stimulus responsiveness for constructing $\mathrm{pH}$ or light responsive channels[100]. These properties come from the possible organic architecture, low energy barrier for isomerization and weak acid/base groups that can protonate or deprotonate in responding to environmental changes. One of the best reasons for large utilization of organic molecules in track-etched nanopore is the simplicity of immobilization. 


\section{WILEY-VCH}

For PET or PC membrane, the abundant carboxylic groups on the surface due to chemical etching give possibility for covalent grafting in a very moderate condition.

Covalent grafting aims to form chemical bonds between surface and target molecules which can form the most stable functionalization. For PET or PC track-etched membranes, amide moieties are usually preferred because the reaction between an amine and carboxyl moieties catalyzed by 1-Éthyl-3-(3-dimethylaminopropyl)carbodiimide (EDC)[101] and NHydroxysuccinimide (NHS)[102] is simple, fast and running in aqueous solution. This allows grafting biological molecules. The mechanism is depicted in figure 2 a, b. Carboxylic acid moieties are activated by EDC to form an o-acrylisourea active ester intermediate; then amine moieties replace EDC to form amides. The EDC is a zero length crosslinking agent[103] which means that no additional chemical structure is introduced during the reaction. Besides amide bonds, it can also catalyze phosphate groups to form phosphoramidate bond with amines[104]. NHS molecules can easily form an NHS-ester derivative which can then react with a carboxylic for amide bond formation. Pre-prepared polymers can be mixed with EDC to activate carboxylic groups and can also be mixed with EDC/NHS to activate both carboxylic and amine groups. The reactions can be either one-step or two-step. One step reaction has a higher conversion rate but the reaction conditions may destroy reactive especially some biomolecules. Two-step reaction has less conversion rates while reactive are added after the activation step in rough conditions. Due to properties of polymer materials, the track-etched membrane may be damaged by several organic solvents which is largely used and necessary in many organic reactions. This more or less limits the rich diversity of organic chemistry in polymer membranes.

\subsubsection{Polymer functionalization}

The functionalization by polymers allows giving new properties to the membrane such as stimulus responsiveness and selectivity. It can involve polymers which cannot be used to fabricate the bulk membranes limited by mechanical stability, for example. Two methods are 


\section{WILEY-VCH}

employed for surface functionalization by stimuli-responsive polymers: "grafting from”[105] and "grafting to"[106]. "Grafting from" is an in situ polymerization from a surface and "grafting to" involves anchoring the already prepared polymer to the membrane surface. One or the other method should be chosen according to the base membrane physical properties, chemical properties (resistance to the solvent, type of surface moieties and their density), the polymerization conditions (solvent resistance) and so on. There is a common difference between "grafting to" and "grafting from". For the "grafting to" methods, the grafted point density depends on the polymer molecular weight: larger the molecule size is, lower the covering rate will be. While for the "grafting from" methods these two factors are less dependent.

\section{"Grafting from" methods}

The "Grafting from" methods for polymer decorations of surface refer to a series of surface initiated polymerization. In the three past decades, the polymerization techniques have undergone a period of active development especially in controllable polymerization such as atom transfer radical polymerization (ATRP)[107] and reversible addition fragmentation chain transfer polymerization (RAFT)[108]. Since their found, ATRP and RAFT have been widely investigated for applications in surface initiated domain[109]. The surface initiated polymerization requires firstly the immobilization of reactive monomers on the surface[110]. Then, the propagation of chains obeys to the same process of the polymerization than in bulk conditions.

Atom transfer radical polymerization (ATRP) utilizes transition metal complexes $(\mathrm{Cu}, \mathrm{Fe}$, $\mathrm{Ru}, \mathrm{Ni})$ as catalysts and initiators of alkyl halides (R-X)[111]. Among them, $\mathrm{Cu}$ complexes are the most used ones. The ATRP reaction begins with the activation of dormant species (R$\mathrm{X})$ by the transition metal which creates alkyl radicals and oxidization of the metal atom. Then, the activated alkyl moieties keep growing with monomers. In addition, the reaction equilibrium is shifted toward the formation of halogen stopped species with low radical 


\section{WILEY-VCH}

concentrations. Because each chain has the same probability to grow up or to form dormant species, the polymers will have similar molecular weights and narrow molecular weight distribution. During the polymerization, as initiators, catalysts and monomers interact to form different intermediate states, the reaction process depends on many factors such as catalyst concentration, initiator concentration, type of ligands and solvent[112].

With abundant organic groups especially carboxylic ones inside many track-etched membranes, ATRP polymerization has been largely applied in their functionalization for designing "smart" membranes[113]. The most famous polymer synthesized by surface initiated ATRP inside polymer membranes is poly(N-isopropyl acrylamide) (PNIPAM) which can change its conformation in responding to temperature change. Demoustier-champagne's group has polymerized PNIPAM inside PET membranes to construct thermo-responsive membranes[114]. This property can be controlled by varying the initial membrane pore size, the polymerization degree and the type of salt in solution[115]. Then, Guo et al. achieved the PNIPAM polymerization in a single PET nanopore revealing thermo-responsive rectification behaviors[116].

Reversible addition fragmentation chain transfer polymerization (RAFT) is also one of the most popular techniques for surface initiated polymers brushes preparation[117]. It requires an initiator which can decompose as free radicals and a RAFT agent which has a form of thiocarbonyl moieties to control the reaction. The reaction begins from the fragmentation of the initiator for radical release. Then monomers undergo a radical polymerization with initiators. The controllable step comes from the participation of RAFT agent to the chain growth. The polymer reacts with RAFT agent to transfer the radical to the alkyl chain in the agent under a reversible process. The new formed radical species will continue to polymerize. From certain size, they will establish the equilibrium with RAFT agent connecting another preformed polymer. This procedure is known as the main RAFT equilibrium. Thus by 


\section{WILEY-VCH}

controlling both temperature and chemical factors, desired polymer size can be obtained with a narrow size distribution.

Clochard's group used RAFT-mediated radical polymerization for grafting poly(acrylicacid) (PAA) inside the nanochannels of track-etched $\beta$-PVDF membranes[118]. This gives polymer brushes with low polydispersity and homogeneous composition inside the channel. Later the same group polymerized poly(4-vinyl pyridine) (P4VP) in PVDF membrane by radiationinduced RAFT[119]. The P4VP functionalization makes the membrane a good sensor for monitoring trace of mercury in water. In this strategy, it is essential to preserve the free radical generate by the track. The storage conditions of the irradiated polymer film have to be appropriate. Other than PVDF membrane, Zdorovets et al. functionalized PET membranes by UV-induced polymerization of PAA and P4VP to design sensors for heavy metal ions detection including copper, lead, and cadmium ions[120].

The above examples show a dynamic and successful transfer from surface polymerization to confined surface polymerization in nanochannels. However, compared to polymerization on a plane surface, there are still such differences under confined space. The diffusion of monomers into nanochannel affected by steric effect of pore walls will impact the polymer growth and so on. This makes it sometimes difficult to get similar results in nanopore as on a plane surface. So, fundamental research can be investigated later on understanding the basic aspect of polymerization in single nanopore. This latter can be a strong platform to study the confinement effect on the dynamics of polymerization reactions. Besides ATRP and RAFT, many other surfaces initiated polymerization have also been developed including nitroxidemediated polymerization[121], anionic polymerization[122], photoiniferter-mediated polymerization[123], ring-opening metathesis polymerization[124] and living ring-opening polymerization[125] which has not been generally applied in track-etched membranes. The future works can be interesting to design new membranes by these abundant polymerization methods in track-etched membrane. 


\section{WILEY-VCH}

\section{"Grafting to" methods}

Pre-prepared responsive polymers can be attached onto a membrane surface by covalent grafting, adsorption and coordination grafting to form self-assembled monolayers (SAMs).

Chemical grafting._As the same as the section 2.2.2, chemical grafting of polymers use the same principle with small organic molecules. Chemical reactions between carboxylic acid and amines catalyzed by EDC/NHS can immobilize polymer brushes inside track-etched nanopores. Compared to "grafting from" methods, chemical grafting of pre-prepared polymers allows preparing polymers separately which can be controlled better than directly in confined space. However, the size of macromolecules is comparable or sometime larger than nanopore. This can limit polymer diffusion into confined space and the grafting density can be relatively low or can be influenced by polymer size.

Chemical grafting polymers in track-etched nanopore have been largely reported in constructing stimuli-responsive ion channels and biosensors. Nguyen et al. immobilized DNA strands in conical PET nanopore catalyzed by EDC to design a voltage-gated ion channels[126]. Nasir et al. functionalized cylindrical PET membrane by amine-terminated PNIPAM brushes to construct membranes with thermally controlled permeation[127]. Ali et al. designed a biosensor by grafting e amine-terminated single-stranded DNA aptamer inside cylindrical PET nanopore for lysozyme detection[43]. In the same year, Lepoitevin et al. used PEG like avidin grafting in an ALD treated PET nanopore. Based on avidin-biotin recognition, this nanopore show both $\mathrm{pH}$-gated and $\gamma$-globulin detection properties[128].

Layer-by-layer deposition. Layer-by-layer adsorption is a kind of bottom-up fabrication method which takes advantages of self-assembling processes. Electrostatic attraction and intermolecular interactions as hydrogen bonds, Van der Waal's forces and specific biological interactions can be driving forces. Layer-by-layer deposition technique (Figure 3 a) is a good method for fabrication[129] or functionalization[130] of the membranes by sequential physical adsorption of complementary macromolecules. This method has the merit to be 


\section{WILEY-VCH}

simple and low cost. As the energy level of physical interaction is relatively low compared to chemical bonds, the physically adsorbed layers can be desorbed by changing $\mathrm{pH}$, ionic strength, solvent or other conditions[131]. This soft and flexible structure thanks to the weak interactions makes it suitable to design stimuli-responsive membranes[40, 41]. However, this is also a disadvantage for the possible undesirable desorption when environment changes. The application of layer-by-layer (LBL) adsorption of polyelectrolytes in track-etched membrane can dramatically change the surface charge density, the pore size and thus the ion distribution inside the pore. According to the properties of polyelectrolytes, these changes in nanopores can be varied in responding to the $\mathrm{pH}$ and ionic strength changes. The LBL deposition has an easy access in PET and PC track-etched nanopore because of the negative charges of carboxylate moieties on the pore wall at $\mathrm{pH}$ greater than 4. Different than on flat surfaces, increments of thickness per cycle of deposition can be much larger and assembly thickness in highly dependent on nanopore size[132]. Layer-by-layer assembly of polyelectrolytes was firstly introduced in single-track-etched nanopore by Ali et al. using Poly(allylamine hydrochloride) (PAH) and poly(sodium 4-styrenesulfonate) (PSS)[133]. Both theoretical and experimental results indicated that the charge inversion process was strongly affected by nanoconfinement. The ionic current rectification and surface charge density decreases with polyelectrolytes layers grown revealing a charge overcompensation. Our group showed the simplicity and reversibility of PLL/PSS functionalization in PET conical nanopore. A negative charge overage was revealed because of the higher charge density of PSS[131]. Based on this, we then improved PET conical nanopore by increasing charge density inside using chitosan (Ch)/PAA couple to enhance osmotic energy harvesting[134].

Self-assembled monolayers coordination. Another kind of widely used grafting is selfassembled monolayers (SAMs) using thiol-Au coordination bonds (Figure $3 \mathrm{~b}$ ). It consists immobilizing thiol or disulfide terminated molecules onto gold substrate[135]. The chemisorption of thiol moieties on gold can form stable Au-S bonds. We mentioned above 


\section{WILEY-VCH}

that "grafting to" methods suffer from low grafting density because of the steric effect of macromolecules. However, the SAMs can offer a very dense layer thanks to inter-molecule forces. Although, it usually takes a long time for obtaining SAMs because of the multi-steps of the process includes physical adsorption, lying formation phase, standing nucleation phase and completion phase. It draws still great interest for researchers for its simplicity and broad applications range. This last convenience comes from the possibility of a pre-step of covering surfaces with gold using electroless deposition, sputtering or vapor deposition on various substrates including polymer membranes[136] and solid-state nanopores[137].

\subsubsection{Protein grafting}

Proteins as a large category of biomolecules play an important role in living systems. They are widely involved in nanopore technology for protein detection and biosensing. They are usually charged, contain a large number of amine groups and have specific structures. These properties make it possible to immobilize proteins inside track-etched membranes via adsorption[138], chemical grafting[139] and specific recognition. Lysozyme, rchymotrypsin[140] and BSA[141] were reported being adsorbed in PET membrane. Specific recognition requires immobilization of first molecules on surface called bio-recognition moiety. Then a strong recognition can happen to another molecule called bio-conjugate. Ali et al. reported p-aminophenyl $\alpha$-Dmannopyranoside (APMP)/ Concanavalin A recognition in bullet-like PET membrane[142]. Nguyen et al. achieved saccharide/glycoprotein recognition inside conical PET nanopores[143]. Ma et al. immobilized hyaluronidase in tip side of conical PET nanopore by chemical grafting in order to measure enzyme-substrate complexion duration between hyaluronidase and hyaluronic acid[139]. Even though many reports achieved successfully the protein grafting, the protein can easily lose its activity after grafting which limits this functionalization method. 


\section{WILEY-VCH}

\section{Transport properties in nanopore}

\subsection{Electrostatics near interface}

\subsubsection{Electric double layer and Poisson-Boltzmann equation}

The transport phenomena through nanopores and nanochannels are interesting because of their small aperture. Indeed, when dimension gets shrinks down to nanoscale size, interfacial phenomena become more dominant than the bulk. Thus the fluid behaviors can be changed in confined space as well as electrochemical properties. In most cases, surface of nanopores and nanochannels can have charges.

The processes of the charge formation, chemical potential and entropy are the driving forces that minimize the Gibbs free energy to reach the equilibrium with outer ions in solution. At the same time, an electric double layer forms near the surface in electrolyte solution (Figure 4). Due to electric attractions of the surface charge, counterions will be closely adsorbed to get a minimized potential. These adsorbed counterions form the Stern layer or the condensed ion layer specifically in polyelectrolyte science. They can induce a screening effect of the polymer charges. Then outer of this counterion layer, the diffuse layer is formed by relatively free ions to neutralize the Stern layer. This electric double layer as shown in figure 5 becomes the essential model to study ion concentrations and charge distributions near the charged surface in nanopore and nanochannels.

The ionic concentration and the distribution of charge density in electrolyte solutions can be described by Poisson-Boltzmann Equation (PBE) which is obtained by means field approximation of the potential of mean force [144]. Poisson's equation in the electrolyte solution system describes the electrostatic potential as a function of the number density of charge and it reads:

$\nabla^{2} \psi(\mathbf{r})=-\frac{4 \pi}{\epsilon} \sum_{i} z_{i} q c_{i}(r)$ 
where $\psi$ is the electric potential, $\epsilon$ is the dielectric constant, $z_{i} q$ is the electric charge of the ion $i, c_{i}$ is the concentration of ion $i$ at position $r$.

In the Gouy-Chapman model of electric double layer theory, the ions close to the surface follow Boltzmann distribution:

$c_{i}(r)=c_{i}^{0} e^{-\beta \omega_{i}(r)}$

where $\omega_{i}(\boldsymbol{r})$ is the potential of mean force of ion $i, c_{i}^{0}$ is the counterion concentration at the point of zero potential, $\beta=1 / k_{B} T$ where $k_{B}$ is Boltzmann constant.

In mean field approximation, if we neglect ion interactions and assume the potential of mean force approximately equal to its electric potential:

$\omega_{i}(\boldsymbol{r}) \cong z_{i} q \psi(\boldsymbol{r})$

we get the Poisson-Boltzmann equation:

$\nabla^{2} \psi(\boldsymbol{r})=-\frac{4 \pi}{\epsilon} \sum_{i} c_{i}^{0} z_{i} q e^{-\beta z_{i} q \psi(\boldsymbol{r})}$

If we define that the Stern layer radius is $a$, the diffusive layer radius is $R$. At the boundary of the Stern layer the surface charge density at $r=a$ is given by $v / 2 \pi a,(v$ is the linear charge density of polyelectrolyte molecules) which gives a first boundary condition:

$\left.\frac{\beta \partial \omega_{i}(\boldsymbol{r})}{\partial r}\right|_{r=a}=\frac{e^{2} v}{2 \pi \epsilon k T a}$

while at the boundary of the diffusive layer, the electric field should vanish, then we get the second boundary condition:

$\left.\frac{\beta \partial \omega_{i}(r)}{\partial r}\right|_{r=R}=0$

If we assume that the surface potential is homogeneous and small $(<25 \mathrm{mV})$ in the whole EDL and use $e^{-\alpha}=1-\alpha$ for $\alpha$, then we get Debye-Hückel approximation:

$\nabla^{2} \psi=\frac{d^{2} \psi}{d z^{2}}=k^{2} \psi(r)$

where 
$\mathrm{k}=\left(\frac{e^{2} \sum_{i} n_{i}^{\infty} z_{i}^{2}}{\epsilon k_{B} T}\right)^{1 / 2}$

which is called Debye-Hückel parameter. For small surface potential, analytical solution can be obtained as:

$\psi(\boldsymbol{r})=\psi_{0} \exp \left(-z / \lambda_{D}\right)$

where $\lambda_{D}=k^{-1}$ is the Debye length characterizing the thickness of the EDL, thus the Debye length is inversely proportional to ion concentrations of solution. For a high surface potential, Gouy-Chapman's model is taken into account:

$\tan h\left(\frac{e \psi(r)}{4 k_{B} T}\right)=\tan h\left(\frac{e \psi_{0}}{4 k_{B} T}\right) \exp \left(-z / \lambda_{D}\right)$

where the condition of equal valence of co-ions and counterions is applied for this result.

As Debye length depends only on ion concentration of the solution, it can be simply expressed as a function of ion concentration $n_{i}$ in the case of a symmetrical electrolyte $\left(z_{i}=z_{i}\right)$ at $25^{\circ} \mathrm{C}:$

$\lambda_{D}=\frac{2.15 \times 10^{-10}}{\sqrt{\frac{1}{2} \sum_{i} n_{i} z_{i}^{2}}}$

For a $\mathrm{KCl}$ concentration of $10^{-5} \mathrm{M}$, the Debye length is as long as $96.3 \mathrm{~nm}$ which create a zone big enough for various electrokinetic phenomena close to interface.

\subsection{Hydrodynamics and Electrokinetics near interface}

\subsubsection{Liquid slippage}

Before discussing the transport of electrically charged ions and molecules under a potential field (EDL), it should be introduced a more common phenomenon named slippage[145] that occurs at liquid/solid interface under frictionless flux (Figure 5). In the case of nanopore, it can play an important role on the pressure drop, electrical and diffusive transport. The characteristic of slippage which has the opposite direction of Debye length is the slip length[146]. It is an extrapolated distance relative to the nanopore wall where the tangential velocity component vanishes as shown in figure 6 . Various factors can influence the slip 


\section{WILEY-VCH}

length including weak wall fluid attraction, surface roughness, high shear rates and nucleation of nanobubbles at hydrophobic surfaces. As it is an interfacial hydrodynamic phenomenon, to find the slip length $b$, we should begin with the liquid-solid friction. The friction force is linear to slip velocity under the form of:

$F_{f}=-\mathcal{A} \lambda \mathrm{v}_{t}$

where $\lambda$ is the friction coefficient and $\mathcal{A}$ is the lateral area.

The slip length is defined as $b=\eta / \lambda$, where $\eta$ is the bulk viscosity. According to this relation, the slip length should be large for a smooth surface especially a hydrophobic surface; this is why a high water transport velocity was reported in carbon nanotubes[147]. The slippage has also an interesting property since it can explain the pseudo-surface charge reported in hydrophobic nanopore[148].

\subsubsection{Electro-osmosis, electrophoresis and streaming potential}

The liquid slippage can occur on charged or non-charged surfaces. If we refocus to a charged surface with an EDL, the flow of liquid containing ions in EDL can involve interesting electrodynamic phenomenon [149]. If the flow is driven by an electric field, both electroosmosis[150] and electrophoresis[151] will happen. If the flow is driven by hydrostatic pressure, a streaming potential will be obtained.

When an electrical field is applied parallel to the interface, from the slip plane, the liquid velocity increases from zero to a maximum value $v$ described by Smoluchowski formula[152]:

$\mathrm{v}=-\frac{\varepsilon \zeta}{\eta} E_{e}$

where $\varepsilon$ is the dielectric constant, $\zeta$ is the zeta potential and $\eta$ is the viscosity. Here we can find a linear relation between the liquid velocity and the applied electric field. This plug-like flow will be changed when the ionic strength is ultra-low with a large Debye length. Using Stokes and Poisson equations, we can develop the $\mathrm{v}_{(z)}$ as: 
$\mathrm{v}_{(z)}=-\frac{\varepsilon \zeta}{\eta} E_{e}\left(1-\frac{\psi(z)}{\zeta}\right)$

where the potential distribution appears as a variable of the velocity.

As an interfacial flow, the electro-osmotic transport will be influenced by slippage which can decrease the friction in EDL. Then as shown in Fig. 11, the hydrodynamic velocity gradient can contain $b+\lambda_{D}$. Thus if we apply $\zeta$ :

$\zeta=v_{0} \times\left(1+\frac{b}{\lambda_{D}}\right)$

into Eq. (16), the velocity at surface $\mathrm{v}_{(0)}$ becomes:

$\mathrm{v}_{(0)}=\frac{\varepsilon}{\eta} E_{e} \frac{b}{\lambda_{D}}$

$\mathrm{V}_{(0)}$ is proportional to the slip length. It shows that surface properties can highly influence electric induced transport and can be optimized for osmotic energy harvesting[153].

Now, if an electric field is applied parallel to the interface, the charged particles will move to form electrophoresis flow[154]. The electrophoresis mobility will depend on the EDL thickness. For thin EDL thickness compared to the particle radius, the electrophoretic mobility can be expressed by Helmholtz-Smoluchowski limit:

$\mu_{e p}=\frac{\varepsilon \zeta}{\eta}$

While for thick EDL, the electrophoretic mobility can be given by Hückel-Onsager limit:

$\mu_{e p}=\frac{2 \varepsilon \zeta}{3 \eta}$

Besides the electric field, the driving force for generating flows can be hydrostatic pressure which leads to the formation of streaming potential across a channel[155, 156]. The mobile ions in the EDL move under pressure as a flow of ions is called streaming current. The accumulation of ions creates an electric field causing an opposite current as conductance one. The streaming potential is the difference of electrical potential along the channel. It can be very useful to experimentally determine surface charge, the surface $\mathrm{pKa}$ as well as the successful of functionalization[156, 157]. 


\section{WILEY-VCH}

\subsubsection{Nernst-Planck equation}

If we focus on transport of charged species like ions without fluid flow, the continuum meanfield theory can be used. Under gradient of concentration, the state of disequilibrium will generate an ion flux including diffusion and migration [158]. It is proportional to the gradient of chemical potential:

$J_{i}(\boldsymbol{r})=-D_{i} c_{i}(\boldsymbol{r}) \nabla \beta \mu_{i}(\boldsymbol{r})$

where $D_{i}$ is the diffusion coefficient and $\mu_{i}(\boldsymbol{r})$ is the chemical potential of specie $i$ at the position $\boldsymbol{r}$. There are two contributions of the chemical potential: the translational entropy and interaction with external electrostatic potential $\psi$ :

$\mu_{i}(\boldsymbol{r})=\mu_{i}^{0}+k_{B} T \ln \left(\frac{c_{i}(\boldsymbol{r})}{c^{*}}\right)+z_{i} q \psi(\boldsymbol{r})$

where $\mu_{i}^{0}$ is the standard chemical potential of specie $i$. The first two terms correspond to chemical potential without interactions and the third term represents the interactions under the electrostatic potential. If we consider only the chemical potential without other interactions, the Fick's first law of diffusion can be written by inserting first two terms of equation (22) into equation (21):

$J_{i}=-D_{i} \nabla c_{i}$

As all other interactions are neglected, the Fick's law can only give flow driven by a concentration gradient [159]. When external forces or other interactions are applied, NernstPlanck equation can be obtained by inserting the entity of equation (22) into equation (21):

$J_{i}=-D_{i}\left(\nabla c_{i}+c_{i} \beta z_{i} q \nabla \psi\right)$

Compared to the Fick's first law, the Nernst-Planck equation may describe diffusing particles under other driven forces such as osmotic gradients, Van der Waals forces and so on. As Poisson's equation describes spatial distribution of the electric potential, combining Poisson and Nernst-Planck known as PNP equation is used widely to calculate current-voltage 


\section{WILEY-VCH}

characteristics, profiles of ionic concentrations and profiles of electric potential in nanopore/nanochannel[160, 161].

\subsubsection{Navier-Stocks equation}

As discussed above, Nernst-Planck equation can describe movement of charged particles under chemical potential gradients and external potential without liquid flows. If a mechanical force is applied to lead to a net solution flow, Navier-Stocks equation should be used. The incompressible momentum Navier-Stokes equation reads:

$\frac{\partial \mathrm{v}}{\partial \mathrm{t}}=-\frac{1}{\rho} \nabla p-(\mathrm{v} \cdot \nabla) \mathrm{v}+v \nabla^{2} \mathrm{v}+\frac{1}{\rho} F$

where $\mathrm{v}$ is the solution velocity, $v$ the kinetic viscosity of fluid, $\rho$ the fluid density and $\mathrm{F}$ the applied force. It includes four terms which is pressure gradients induced force, inertial forces, viscous forces and external forces in order. It should be stated that in nanopore/nanochannels, as the length scale is very small in nanometer range, the effect of viscous forces can be much larger than that of inertial forces. The third term can be neglected. Thus, we can add the net flow described by Navier-Stocks equation into equation (24):

$J_{i}=-D_{i}\left(\nabla c_{i}+c_{i} \beta z_{i} q \nabla \psi\right)+c_{i} \mathrm{~V}$

Finally, by combining Poisson's equation, Nernst-Planck equation and Navier-Stokes equation (PNP-NS), one can calculate and obtain satisfied model results near charged interface[162, 163].

\subsection{Transport in nanofluidic}

\subsubsection{Donnan and membrane potential}

Until this section, we discussed essentially the interfacial transport phenomenon with or without a charged surface. When the nanopore radius is of the same order as Debye length, the overlap of EDL will happen. Thus, the whole space in the nanochannel will be influenced by the surface charge. Under this condition, a nanochannel with charged surfaces (positive or negative) in an electrolyte solution will be selective to counterions. To reach the 
electrochemical equilibrium between outside and inside of the channel with different ion species concentrations, an electrical potential can be found so-called Donnan potential (Figure 6 a):

$\Phi_{D}=\frac{R T}{z_{i} F} \ln \left(\frac{c_{i}^{I}}{c_{i}^{I I}}\right)$

where $c_{i}^{I}$ and $c_{i}^{I I}$ are ion concentrations outside and inside the channel.

If the channel connects two reservoirs with different salt concentrations which is a common case for membrane utilization during desalination and reversed electrodialysis energy harvesting, the membrane potential can be calculated by combining two Donnan potentials $\Phi_{D 1}, \Phi_{D 2}$ of the two membranes sides and the diffusion potential $\Phi_{\text {diff }}$ (Figure 6 b):

$\Phi_{m}=\Phi_{D 1}+\Phi_{D 2}+\Phi_{\text {diff }}$

where $\Phi_{\text {diff }}$ is calculated as:

$\Phi_{\text {diff }}=-\frac{t_{c a}^{I I}}{z_{c a}} \frac{R T}{F} \ln \frac{c_{2_{c a}}^{I}}{c_{1_{c a}}^{I}}+\frac{t_{a n}^{I I}}{z_{a n}} \frac{R T}{F} \ln \frac{c_{2_{a n}}^{I}}{c_{1_{a n}}^{I}}$

where $t_{c a}^{I I}$ and $t_{a n}^{I I}$ are the transference numbers of cations and anions. Thus from Eq. (28) and Eq. (29) we can obtain the membrane potential as:

$\Phi_{m}=\frac{R T}{F}\left(\ln \frac{c_{1}^{I I} c_{2}^{I}}{c_{1}^{I} c_{2}^{I I}}-\frac{t_{c a}^{I I}}{z_{c a}} \ln \frac{c_{2 c a}^{I}}{c_{1}^{I}}+\frac{t_{a n}^{I I}}{z_{a n}} \ln \frac{c_{2 a n}^{I}}{c_{1 a n}^{I}}\right)$

\subsubsection{Perm-selectivity and concentration polarization}

As the counterions of the charged surface are the majority in the EDL, when Debye length is large (typically at low salt concentration), an enrichment of counterions occurs in the channel and a corresponding exclusion of co-ions will also occur. This exclusion-enrichment effect generates perm selectivity of membranes for diffusive ion transport [158]. The effective diffusion coefficient is defined as:

$J_{ \pm}=-D_{\text {eff }} \frac{\Delta \rho}{L}$ 
where $J_{ \pm}$is the ion flux, $\Delta \rho$ is the concentration drop along nanochannel and $L$ is the nanochannel length.

Other than asymmetric concentration, an electric field applied across a nanochannel in an electrolyte solution can generate ion movement and reorganization of ions. As the surface charge promotes the presence of the counterion, the applied electric bias can support a larger current inside the pore than the entrance. Such a counterion depletion will occur at the entrance and a counterion enrichment will occur at the exit (Figure6 c). This concentration polarization in responding to large applied electric bias breaks the linear current-voltage relationship and leads to a ionic current rectification that will be talked latter.

\subsubsection{Nanochannel conductance}

Ion enrichment and ion depletion are a consequence of ions flows. Other than that an electric potential applied to a nanochannel immersed in electrolyte solution generates an electric current. Thus, the channel can be considered as a resistance and inversely the conductance can be defined to a channel as the ratio of electric current to electric potential drop. The ionic conductance has two contributions from the bulk solution and the electro-osmosis in EDL[164] (Figure 7). It can be expressed for a negatively charged nanochannel in a monovalent electrolyte solution as:

$G=\left(\mu_{+}+\mu_{-}\right) e C \frac{S}{L}+2 \mu_{+} \sigma_{s} \frac{w}{L}$

where $\mu$ is the mobility, $C$ is the ion concentration, $S$ is the cross section of channel, $w$ is the channel width, $L$ is the channel length and $\sigma_{s}$ is the surface charge density.

Recently, Balme et al. proposed a hybrid approach considering Debye length, advection, good co-ion exclusion and electro-osmosis[165]:

$G_{h y b}=\frac{\pi R^{2} \kappa_{h y b}}{L}$

$\kappa_{h y b}=e^{2} c_{s}\left(\mu_{+}+\mu_{-}\right) \sqrt{1+\left(\frac{\sigma}{e R c_{s}}\right)^{2}}+\frac{e|\sigma|}{R}\left(\mu_{+}-\mu_{-}\right)+\frac{\sigma^{2}}{2 \eta} f\left(\sigma^{*}\right)$ 
where $c_{S}$ is the bulk concentration, $R$ the pore radius, $\sigma$ the surface charge density and $f\left(\sigma^{*}\right)$ a monotonous decline function which describes the correction for the large surface charge for the advective part of "Good Co-ion Exclusion" approximation:

$\sigma^{*}=\pi l_{B} R \frac{\sigma}{e}$

where $l_{B}$ is the Bjerrum length.

It's also possible to consider the slip length as:

$\kappa_{\text {slip }}=\kappa_{\text {hyb }}+\delta \kappa_{\text {slip }}=\kappa_{h y b}+\frac{2 \sigma^{2} b}{\eta R}$

where $b$ is the slip length.

At high electrolyte concentrations, the conductance is contributed by ions in bulk solution inside the channel; while at low electrolyte concentrations, counterions in diffuse layer of EDL contribute essentially. When the two terms equal, a concentration $C_{t}$ can be found as $0.5 C_{e}$, where $C_{e}$ is the concentration of excess mobile counterions.

\subsubsection{Ion current rectification}

Until now, all considered nanochannels have a symmetric geometry and a homogeneous charge distribution. It means that under a positive or negative voltage (opposite direction), ionic current remains the same. While if we break the symmetry by considering nanochannel with dissymmetrical shape, heterogeneous distributed surface charge or concentration gradient, the direction change of electric bias will cause a current change known as ion current rectification (ICR) [37, 166].

\section{Geometry}

When a nanochannel has an asymmetric shape such as conical, bullet-like, funnel geometries, the consequence is that the different cross sections of the two sides cause different conductance[167]. For a conical channel, if the voltage drives counterions from the large side (base) to the small side (tip) (Figure 8 a), the quantity of counterions entering in channel is 


\section{WILEY-VCH}

larger than that getting out because of the different cross section area[168]. Thus an accumulation of counterions appears inside the channel close to the tip. To ensure the electroneutrality, co-ions will also accumulate in the same region. So both counterions and coions concentrations increase at tip which leads a global augmentation of channel conductance standing called open state. If the voltage is inverted (Figure $8 \mathrm{~b}$ ), with the same principle, ion depletion will take place in the channel inducing a lower conductance called a closed state[51, $160,169,170]$.

There are several asymmetric geometries including cone, funnel, bullet, etc. Different geometries can show different rectification behaviors with varying pore diameters, pore length, cone angle and so on. Ramirez et al. studied the rectification factor as a function of tip diameter and nanochannel shapes using continuum approach based on the Nernst-Planck equations[171]. By fixing base side diameter, pore length and electrolyte concentrations, results of calculation demonstrate that rectification factor increases when tip diameter decrease. With the same electrolyte concentration meaning the same EDL thickness, tip side with a smaller diameter can be more dominated by EDL leading to higher cation selectivity[172]. Then, by controlling other conditions but varying shape parameters, it is presented that bullet-like nanochannel can have a higher current rectification and a higher conductance than conical shape[133]. Pietschmann et al. studied influence of pore length on current rectification using 1D reduction of the PNP model with the Donnan equilibrium values for boundary conditions[173]. By increasing pore length with fixed tip diameter and cone angle, an enhancement of electrolyte concentrations was observed. The increase of negative current and decreasing of positive current lead to an amplification of the rectification factor. Tseng et al. investigated influence of nanopore cone angle on current rectification behaviors theoretically[174]. Two methods were compared: one fixed tip diameter and the other one fixed base diameter. When tip diameter is fixed at $5 \mathrm{~nm}$, the ICR presents a maximum value. The increase of electrolyte concentration under negative voltage and 


\section{WILEY-VCH}

insensitivity to angle variation under positive voltage make rectification factor increase with decreasing cone angle when the angle is small. While for a larger angle, EDL will dominate more space in the pore leading to a higher concentration of counterions. This concentration enhancement with increasing angle leads to a higher rectification factor. Thus a maximum rectification can be observed. If they change tip diameter by fixing a base diameter at $540 \mathrm{~nm}$, a monotonically increasing tendency can be obtained by increasing cone angle up to $15^{\circ}$. A high pore angle can generate a significant ion accumulation under negative voltage and ion depletion under positive voltage leading to increased rectification.

\section{Surface charge}

In the case of asymmetric surface charge distribution, the current rectification occurs due to ion accumulation and ion depletion[175]. An example is shown in figure $8 \mathrm{c}$ and d, a cylindrical channel has positive charges on the left and negative charge on the right. When a positive potential is applied on the side of negative charges (Figure $8 \mathrm{c}$ ), both cations and anions can enter in the channel from two directions. However, they have difficulties to get out because of the opposite surface charges compared to their entrance. They enter in the channel as counterions and become coions when they continue to move out. An accumulation of the anions and cations occurs, and thus, the pore will have an open state. Conversely, when the applied voltage is opposite, both cations and anions have difficulties to enter facing a large electric repulsion (Figure $8 \mathrm{~d}$ ). The ion depletion that occurs in channel leads to a closed state. The surface charge induces ICR that depends on both the density and the distribution of the surface charges. They are determined by surface group nature, density and solution property such as $\mathrm{pH}$. As current rectification is a reflection of ion selectivity, local charge inversion on the pore surface can dramatically change ICR and usually can generate an inversion of I-V curves in conical shape $[41,133,176]$. Several studies based on PNP equations demonstrate a local maximum rectification factor for a defined unipolar conical nanopore by varying surface 


\section{WILEY-VCH}

charge density[173, 174]. In a negatively charged nanopore, the average ion concentration in the channel is insensitive to charge density when charge density is low. It increases under $+1 \mathrm{~V}$ but can increase with increasing charge density. This is contrary when charge density gets higher than a certain value. The average ion concentration becomes independent of charge density for $-1 \mathrm{~V}$ and increases with increasing charge density for $+1 \mathrm{~V}$.

Besides unipolar nanopore, rectification behaviors of bipolar nanopores were also reported[177]. Singh's group theoretically investigated the effect of surface charge density on bipolar cylindrical nanopore[178] and bipolar conical nanopore[179]. In a cylindrical nanopore of $4 \mu \mathrm{m}$ of length and $6 \mathrm{~nm}$ of diameter, positive surface charge and negative charge cover each a half of the pore. Rectification under $\pm 2.4 \mathrm{~V}$ is increased by increasing surface charge density until $20 \mathrm{mC} / \mathrm{m}^{2}$ in solution of $100 \mathrm{mM}$. It is also seen that smooth surface charge gradient can significantly enhance the degree of rectification due to increase in the unipolar character of electrolytes and decrease in reverse ion current. In a bipolar conical nanopore $\left(D_{\text {tip }}=10 \mathrm{~nm}, D_{\text {base }}=50 \mathrm{~nm}, \mathrm{~L}=5 \mu \mathrm{m}\right)$, rectification factor show a monotonically increasing relationship with increasing charge density until $20 \mathrm{mC} / \mathrm{m}^{2}$ in solutions ranging from $5 \mathrm{mM}$ to $50 \mathrm{mM}$. This is attributed to the increase of ion concentration inside the pore when electro-osmotic flow (EOF) is not considered. The EOF related rectification change will be talked later.

Besides surface charge density, the ratio between the length of positively charged zone and negatively charged zone is recently found to be able to change ICR in a bipolar conical nanochannel[180]. Polyelectrolytes modification depth controlled by functionalization time tunes dramatically the rectification factor. Experimentally in a typical $6 \mu \mathrm{m}$ PET track-etched nanopore with tip diameter at about $10 \mathrm{~nm}$, a maximum rectification factor is found with functionalization time of $80 \mathrm{~min}$. The numerical simulation found a maximum for a modification depth of $50 \mathrm{~nm}$. 


\section{WILEY-VCH}

Electroosmotic flow (EOF)

Besides surface charge distribution and geometry, asymmetry in ion concentration can also generate ion current rectification in nanopore/nanochannels. The electroosmotic flow induced ion current rectification has been reported in conical nanopores theoretically and experimentally[170,181] and in conical micropores[150]. Figure 8 e and $f$ show principles of EOF induced ICR. There are two ion flows inside the nanopore: one diffusion flow due to the concentration gradient has always a direction from concentrated part to dilute part; the other one, migration flow caused by electrical bias change directions according to applied bias. Thus, an increase of ion concentration will occur when two flows have the same direction, otherwise ion depletion occurs causing a weaken current. To study rectification due to EOF by simulation based on a continuum model, it is necessary to combine PNP equations with NS equations. This can make clear different results between calculations using PNP equations and PNP-NS equations especially for high voltages[162] .

\section{Other parameters influencing ICR}

Beside the nanopore intrinsic properties (surface charge and shape), many other parameters can influence ICR. These parameters usually relate to properties of electrolyte solutions, external forces, etc. It includes ion valence[162, 182], anions[183], pressure[184], scan rate[185], ionic liquids[186], solvents[187], wettability[116, 188], temperature[189] etc. They can also co-influence ICR by combining several parameters which makes it sometime complex to fully understand ion transport properties.

As the rectification can be easily measured using an amplifier and can be influenced by a variety of parameters, it is thus a very useful signal output for characterizing of ion-sensing, molecule-sensing, stimulus responsiveness, etc. in a channel. 


\section{WILEY-VCH}

\section{Application}

\subsection{Stimuli-responsive ion channels}

One way to provide responsiveness properties to track-etched nanopores is the grafting of polymers that can change their charge density, effective thickness and/or structural conformations in responds to external stimuli such as $\mathrm{pH}$, electrical potential, UV-Vis light and temperature.

\subsection{1. $\mathrm{pH}$ responsive channels}

The $\mathrm{pH}$ responsiveness is the easiest properties to reach for a track-etched nanopore. Indeed, the surface charge is at the origin of ICR and thus the open or close states of the nanopore. After chemical etching, PET exhibits carboxylate moieties on its surface. This means that the ICR is avoided for $\mathrm{pH}$ below $3[37,190]$. Hsu et al. theoretically revealed the charge effect on a bullet like nanochannel with tunable zwitterionic surface[191]. By varying $\mathrm{pH}$ higher or below pKa of surface groups, the surface can be charged positively or negatively. Then in EDL dominated by surface charges, ion current rectification can be changed in responding to surface charge variation. Such open and close states determined by voltage direction can be yielded in asymmetric nanochannels.

In other to improve such properties numerous approaches were investigated using small zwitterionic molecules as well as polyelectrolytes. Pérez-Mitta et al. decorated a polydopamine (PDOPA) coated PET bullet-shaped nanochannel by small molecules 3aminobenzylamine (ABA) for a $\mathrm{pH}$ responsive nanochannel[192]. Zwitterionic behavior of PDOPA can be shown from the inversion of IV-curves in responding to $\mathrm{pH}$ changes. This behavior is more pronounced when the PDOPA layer is decorated by ABA molecules. The functionalization using polyelectrolyte was the most investigated to obtain $\mathrm{pH}$ responsive track-etched nanopores. Typically, the polyelectrolytes exhibit $\mathrm{pH}$ sensibilities with weak cationic or anionic groups or both. The $\mathrm{pH}$ responsive ion gates based on steric effect of polymer brushes by changing charges have been widely reported. Xia et al. grafted DNA 


\section{WILEY-VCH}

brushes inside of PET conical nanopores by EDC/NHS chemistry[193]. At pH 4.5, DNA molecules take a dense packed rigid i-motif structure which has a larger effective size. While at $\mathrm{pH} 8.5$, the DNA brushes get more charges and turn into a loosely packed single strand. This transition reduces the effective size of DNA molecules and thus the pore diameter change is confirmed by an enhancement of the ionic conductance.

Yameen et al. used hydrophilic/hydrophobic effect of weak cationic polymer brushes (poly (4-vinyl pyridine) (P4VP))[194] (Figure 9 a) and zwitterionic effect of polyampholytes brushes[195] (poly(methacrloyl-L-Lysine) (PMALys)) (Figure 9 b) to construct different ion channels. The P4VP brushes were grafted in a cylindrical PET nanopore. At $\mathrm{pH} 2$, the nitrogen atom of pyridine gets a proton to be positively charged. With the electro-repulsion, hydrophilic polymer chains take extended configuration to reduce the pore diameter. When $\mathrm{pH}$ is greater than 5.0, pyridine is deprotonated to be hydrophobic that makes polymer chains fold on the pore surface to spatially open the pore. PI nanopores functionalized by PMALys tune ionic transport by different surface charges in different $\mathrm{pH}$ [195]. As PMALys has both carboxylic groups $(-\mathrm{COOH})$ and amine groups $\left(-\mathrm{NH}_{3}\right)$, at different proton concentrations, the polymer charge can be positive, negative or neutral. The different charged states modulate the selectivity of the channel. The same principles by using polyampholytes to design $\mathrm{pH}$ responsive ion channels were widely reported by several groups for PC conical nanopores[196, 197]. The amphipol containing carboxylic acids and tertiary amines were immobilized on the tip side of conical nanopore which is previously coated by a gold layer[197]. Positive charges of amines in acidic condition give the pore selectivity to anions. Conversely, negative charges of carboxylic acid in basic solution makes nanopore selective to cations. The same polyampholites functionalization was also performed by direct polymerization of poly(3-aminephenylboronic acid) inside the pore[196]. More interestingly, because of negative charges due to the binding between boronic acids and cis-diols, this channel selectivity can be modulated by fructose. The pore is more selective to cations under 


\section{WILEY-VCH}

higher monosaccharide concentrations. Hou et al. grafted PAA brushes inside one side of an hourglass-like single PET nanochannel for $\mathrm{pH}$ responsiveness[198]. Due to the intramolecular hydrogen bonds among the carboxylic acid groups in the polymer chains at the $\mathrm{pH}$ is below $\mathrm{pKa}$, the PAA chains adopt a coil conformation resulting in a close state. When $\mathrm{pH}$ is higher than $\mathrm{pKa}$, the intermolecular hydrogen bonds between PAA chains and water molecules straighten polymers leading to an open state.

Another method was proposed to design $\mathrm{pH}$ responsive ion channels by using layer-by-layer deposition of polyelectrolytes in single nanopore[40] (Figure $9 \mathrm{c}$ ). The layer-by-layer embedded polyelectrolytes introduce both charges and swelling/deswelling properties. By alternating deposition of polyethylenimine (PEI) and chondroitin sulfate (ChS), a pH and ion strength double controlled channel was obtained. At low salt concentrations, the channel is closed at $\mathrm{pH}>4$ because of the charge compensation. At $\mathrm{pH}<4$ the nanopore is open and selective to anions because of the global positive charge. At high salt concentrations, the swelling of the polyelectrolytes makes the pore not responsive to $\mathrm{pH}$ anymore. With similar method, we constructed another nanochannel by poly-L-lysine (PLL)/poly(acrylic acid) (PAA) with and without crosslinking using EDC and PEI/PAA in conical nanopore[41]. Results showed different behaviors between PLL/PAA and PEI/PAA functionalization as PEI/PAA function opens the nanochannel at $\mathrm{pH} 7$ and closes at $\mathrm{pH} 3$ while PLL/PAA function gives no change in changing $\mathrm{pH}$ from 7 to 3 . This indicates a swelling effect of polyelectrolytes in controlling ion transport in such a nanochannel. To confirm the effect of swelling, PLL/PAA functions were crosslinked using EDC reaction. An IV-curve inversion was shown by changing $\mathrm{pH}$ from 7 to 3 leading to a zwitterionic behavior from simultaneous negative and positive charges of PLL/PAA.

\subsubsection{Potential responsive channel}

As ion channels play an essential role in regulating cell membrane potential, biomimetic potential responsive nanopore draw interests of researchers. Buchsbaum et al. reported a 


\section{WILEY-VCH}

DNA-modified conical nanopore as $\mathrm{pH}$ - and voltage-responsive ion gates[199]. The DNA molecules have uniform negative charges at $\mathrm{pH} 5.5$ while they became zwitterionic electrostatic mesh at $\mathrm{pH} 8$. As shown in figure 10 a, negatively charged DNA functionalized conical nanopore does not obey the usual behavior. Indeed according to the surface charge, the current should be lower at positive voltages than at negative voltages because of the ion selectivity. This phenomenon was explained as at a positive voltage, negatively charged DNA strands are deflected to the base side which opens the pore. While at negative voltages, the inverse movement of DNA molecules blocks the pore. This movement of the long charged polymer chain provides a possibility of potential direction sensing. A similar potential responsive channel was reported by Nguyen et al. using conical nanopore functionalized with DNA[126]. In this work, two states have been observed at negative electrical bias. Under moderate voltage around $2 \mathrm{~V}$, ionic concentration become higher reducing the DNA's extension; while at high voltage around 3 to $4 \mathrm{~V}$, DNA molecules become extended by high electric field leading to a small current.

Perez-Mitta et al. developed a series of potential field effect ionic diodes by using conducting polymers. Two strategies were used, one by electrochemical polymerization of polyaniline (PANI)[200] and the other by coating poly(3,4-ethylenedioxothiophene) (PEDOT)[201] layer (Figure $10 \mathrm{~b}$ and c). Both of them use the electric conducting properties of polymers with conjugated double bonds in oxidation state. For PANI under original form, low proton binding degree equals to a low charge density. Under $0.2 \mathrm{~V}$, the PANI is under oxidized form giving a highly positive charge density to the nanopore. This transition makes the pore surface more hydrophilic and a higher conductance is observed. PEDOT has a similar behavior while two oxidation states are found to occur with an external potential of $0.2 \mathrm{~V}$ and $1.6 \mathrm{~V}$ by cyclic voltammetry. The oxidation creates an electron vacancy as a default (p-type) in semiconductors which contribute to the electron conducting known as anion doping. Conversely, a 


\section{WILEY-VCH}

reduction state under $-0.5 \mathrm{~V}$ makes the polymer as n-doping or cation doping. These oxydoreduction changes were characterized by $\mathrm{I}-\mathrm{V}$ dependence.

\subsubsection{Light $U V$-Vis responsive channel}

The transformation of visible light into electrical nerve signal makes Mammalia animals able to perceive the world by eyes. Rod and cone cells in the retina are involved in a vision cycle with the help of numerous photon sensitive ion channels controlled by retinol/retinal. Many studies based on nanopores were designed to mimic such light-responsive channel based on photo-switchable molecules like coumarin[202], azobenzene[203], spiropyran[204, 205] etc. The combination of these photo-switchable molecules with polymers was also investigated in track-etched nanopores[206].

Li, et al. combined azobenzene with DNA molecules as nanopore functions[207]. As shown in figure 11, chemically modified DNA chains by azobenzene are immobilized on the surface of conical pore by Au-thiol chemistry. Under visible light, azobenzene molecules adopt a trans conformation which makes the DNA strands collapse into hairpin structure. Thus the effective pore diameter is enlarged as characterized by a high ionic conductance. While under UV light, the cis-azobenzene helps DNA to recover the relaxed, extended form. This leads to a closing state of the channel. Therefore by changing irradiation wavelength between visible light and UV light, the pore can open and close to modulate the transport. This photo switchable molecule-polymer cooperative control can amplify the difference between closure and opening state. This signal difference between two states is much higher compared to only photo switchable molecule functionalized channels where they are gated by photo-induced surface wetting/dewetting[205] or surface hydrophobicity/hydrophilicity[208]. Also using azobenzene as photo-switchable molecule, Qian et al. proposed a 3D light responsive gating by inserting metal organic frameworks into bullet-like PET membranes[209]. UiO-66 crystals containing octahedral and tetrahedral cavities $(\sim 12 \AA$ and $\sim 9 \AA$ ) connected by $\sim 6 \AA$ triangular 


\section{WILEY-VCH}

windows were synthesized at the tip side of the nanopore. The azobenzene molecules were situated inside the cavities can then change the nanopore between open state and close state in responding to UV/Vis light. As azobenzene molecules are largely dispersed in the MOFs, an on-off ratio of about 18 is obtained.

Besides azobenzene, spiropyran as a photo-switchable molecule is also used for UV-Vis responsive channel design[205]. Zhang et al. functionalized single conical PET nanochannel by chemically grafting spiropyrans[208]. Under visible light spiropyran has no charge which gives hydrophobicity to the pore inner surface. Under UV light, spiropyran isomerizes into merocyanine which has zwitterionic property containing two charges leading to a hydrophilic surface and an open state. Because of zwitterionic property of merocyanine, the nanopore can show opposite ion selectivity in acid and basic solutions. Ma et al. went further in combining spiropyran molecules with polyethylene glycol (PEG) chains[100]. This last one yields a possible self-assembly in visible light which closes the channel by both charging and steric effect (Figure $11 \mathrm{c}$ ). Under UV light, the pore is open because the PEG chain collapse on the pore wall and the charges of merocyanine can also respond to $\mathrm{pH}$ changes. Even spiropyran based light responsive ion channel has been constructed in different configurations; it suffers until now the difficulty to switch from hydrophobic form into zwitterionic state which requires solvent changes. Future research can be focused on this subject in offering a less polar environment to achieve reversible gating completely in aqueous solution.

Other than UV-Vis light control, malachite-green derivatives were used to construct ion channels that can change ion permeability and selectivity in between UV light and darkness[210]. With similar principle of spiropyran, malachite green can dissociate to give a positive charge in UV light or in acidic $\mathrm{pH}$. While the no-charging form in darkness makes ion channels less rectification behavior and less permeable. 


\section{WILEY-VCH}

\subsubsection{Temperature responsive channel}

Temperature sensibility is one of the life-being ability. Biomimetic thermal controllable ion channels based on track-etched nanopore functionalized with polymers can be designed according to two strategies. The first one involves temperature sensitive polyelectrolytes functions and the second one thermal controlled host guest interaction.

As described before, certain polyelectrolytes can react to temperature change by showing conformation variations. The most studied is poly( $\mathrm{N}$-isopropyl acrylamide) (PNIPAAM) that has a phase transition from a swollen hydrated state to a shrunken dehydrated state at lower critical solution temperature (LCST) about $32^{\circ} \mathrm{C}[211]$. This transition can be used to modulate ion transport through the nanopore. Nasir et al. covalently immobilized PNIPAAM chains in a cylindrical and a conical nanopore membrane[127]. The two polymer states were characterized by ionic transport velocity of ionic molecules containing methylviologen $\left(\mathrm{MV}^{2+}\right)$ and 1, 5-naphthalenedisulfonate $\left(\mathrm{NDS}^{2-}\right)$. After PNIPAAM modification a clear increase of both ionic molecules transport rate was observed for both two nanopore geometries while it is more accented for conical pores. At low temperature, PNIPAAM chains behave like extended brushes which decrease the pore size; at high temperature, a pore opening happens as PNAPAAM becomes more hydrophobic and collapse to the surface. A very good reversibility was also tested by changing alternately the temperature between $39^{\circ} \mathrm{C}$ and $23^{\circ} \mathrm{C}$. Then with the same thermal control principle, Liu et al. used azo-PNIPAAM and azo-poly-L-lysine (azo-PLL) to decorate a 3-amino-3-deoxy- $\alpha$-cyclodextrin $(\alpha-C D)$ modified cylindrical nanopore by azo/ $\alpha$-CD host guest interactions[212]. The latter is ultra-stable for a $\mathrm{pH}$ range from 4 to 10 and for a temperature range from $25^{\circ} \mathrm{C}$ to $40^{\circ} \mathrm{C}$. Because of the bifunctionalization of PLL and PNAPAAM, the ion transport can be modulated by both $\mathrm{pH}$ and temperature changes. Polystyrene-block-poly(N,N-dimethylaminoethylmethacrylate) (PS14000-b-PDMAEMA3600) is another thermo-sensitive polymer. It was used to functionalize track-etched membrane by Zhang et al.[213]. As shown in figure 12 a, by 


\section{WILEY-VCH}

measuring I-V curves, a LCST was found between $45^{\circ} \mathrm{C}$ to $50^{\circ} \mathrm{C}$ for the membrane undergoing swelling in ethanol and drying.

Besides thermal sensible polyelectrolytes, another strategy was proposed by Wang et al. using host guest interactions to construct temperature-sensitive artificial channels[214]. The inner wall of nanopore was modified by ionic liquid molecules (IL). As shown in figure $12 \mathrm{~b}$, an inversion of current rectification appears when a pillar[5] arebe (P5A) molecules self-assemble at $25^{\circ} \mathrm{C}$ onto the IL molecules by inversion of the surface charge from negative to positive. When the temperature rises to $55^{\circ} \mathrm{C}$, the departure of the guest $\mathrm{P} 5 \mathrm{~A}$ molecule happens. The anionic transport channel returns to a cationic transport channel. This thermal energy controls host guest self-assembly interactions.

\subsection{Ion gate}

On cell membranes, ion channels are extremely selective for transporting corresponding ions. These ion channels inspired researcher to develop ion sensing channels which can modulate their ion transport behaviors with presence of specific ions. The principle is that ions should bind to the channel surface specifically. To achieve this purpose, five strategies were investigated in track-etched membranes including (i) ion binding by crown ether (ii) ion binding by DNA, peptide or polyelectrolyte chains (iii) ion involved construction/destruction of a macrostructure (iv) ion-selective hybrid biological/polymer nanopore and (v) ionselective hybrid/metal-organic frameworks/polymer nanopore.

\subsubsection{Crown ether cage approaches}

Ethylene glycol crown ether containing several repeat units of -(O-CH2-CH2)- can form a cavity cage which has a comparative diameter with alkali metal ions (Figure 13). The electron-rich oxygen atom on the circle permits the binding of positive ions. With these two properties crown ethers become one of the best molecules to bind alkali metal ions. More excitingly, 4'amninobenzo-18-crown-6 (4-AB18C6), 4'amninobenzo-15-crown- (4-AB15C5) 


\section{WILEY-VCH}

and 4'amninobenzo-12-crown-4 (4-AB12C4) have the corresponding size for $\mathrm{K}^{+}, \mathrm{Na}^{+}$and $\mathrm{Li}^{+}$ respectively.

All of them were achieved being grafted inside track-etched nanopore. For $\mathrm{K}^{+}$sensing, the 4AB18C6 was achieved grafted in three pore geometries. Perez-Mitta et al. grafted 4-AB18C6 by EDC/NHS chemistry in a bullet-like nanopore[98]. The I-V curves measured in $0.1 \mathrm{M} \mathrm{KCl}$ and $\mathrm{NaCl}$ solutions showed a $\mathrm{K}^{+}$driven rectified anions transport which means a stronger binding effect for $\mathrm{K}^{+}$than $\mathrm{Na}^{+}$. The same crown ether was studied by $\mathrm{Wu}$ et al. in a funnellike nanopore[99]. The same behavior was reported. $\mathrm{K}^{+}$binds to $4-\mathrm{AB} 18 \mathrm{C} 6$ at voltages below $3 \mathrm{~V}$. Upper than that, the departure of $\mathrm{K}^{+}$from crown ether occurs. This $\mathrm{K}^{+}$binding/voltage cleaning was confirmed with a good reversibility. The conical nanopore functionalized with 4-AB18C6 and 4-AB15C5 was reported for $\mathrm{K}^{+}$and $\mathrm{Na}^{+}$sensing with a minimum sensitivity about $1 \mathrm{nM}$ [215]. The reversibility of this experiment was achieved by using [2.2.2]-crypt and which has a higher binding energy with cations than the crown ether of 4-AB18C6. For smaller alkali ions, Ali et al. achieved the $\mathrm{Li}^{+}$detection using in a conical pore functionalized with 4-AB12C4[216]. The 4-AB12C4 binds much better of $\mathrm{Li}^{+}$than others monovalent cations including $\mathrm{K}^{+}, \mathrm{Na}^{+}, \mathrm{Rb}^{+}$and $\mathrm{Cs}^{+}$because of its cavity size. Beside crown ethers, other molecules with similar structures were also reported like bis-podand for $\mathrm{K}^{+}[217]$ and $\mathrm{p}$-tertbutylcalix[4]arene-crown (t-BuC[4]C) for $\mathrm{Cs}^{+}[218]$.

\subsubsection{Polymer approaches}

DNA strands peptides and polyelectrolyte with specific binding sites can also capture specific cations. Hou et al. investigated a G-quadruplex DNA binding of $\mathrm{K}^{+}$in track-etched nanopore[219]. As shown in figure 14, the G-rich DNA chains chemically immobilized in the conical pore can combine $\mathrm{K}^{+}$ions to form a cuboid confined space. This $\mathrm{K}^{+}$-induced structure formation is confirmed by an unusual low current level at $2 \mathrm{~V}$ for a concentration from 500 $\mu \mathrm{M}$ to $750 \mu \mathrm{M}$ compared to $\mathrm{Li}^{+}$. At this range of $\mathrm{K}^{+}$concentration, the $\mathrm{G}$ structure makes DNA brushes dense decreasing the pore size. The same group also investigated nanochannel 


\section{WILEY-VCH}

functionalized with peptides and single-stranded DNA. The binding ability comes from the specific sites along polymer chains. The peptide chains contain $\mathrm{Cys}_{2} \mathrm{His}_{2}$ residues that can capture $\mathrm{Zn}^{2+[220]}$ while the DNA chains that contain an ion-specific thymine-thymine (T-T) base can form stable $\mathrm{T}-\mathrm{Hg}^{2+}-\mathrm{T}$ complexes[221]. The later complex can be broken by cysteine addition.

\subsubsection{Reversible self-assembly of macro structure}

Another strategy to detect ions is the reversible self-assembly of a macro structure inside nanopore thank to the presence of ions. Such macro structure controlled by ions can be

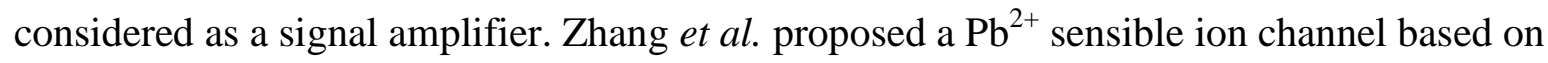
DNAzyme[222] (Figure 15 a). Briefly, DNAzyme molecules containing DNA chain and RNA chimera where an adenine nucleoside (rA) is the cleavage site was immobilized from the base side of conical pore by Au-thiol chemistry. The long chain DNA molecules decrease the effective pore size which corresponds to a closed state. When $\mathrm{Pb}^{2+}$ ions are added, the DNA chains are cleaved into two short fragments at the rA site. This degradation effect of $\mathrm{Pb}^{2+}$ on DNA long chain will destroy the channel gate that leads the pore to an open state. A similar approach was proposed by Lou et al.[223]. In a cylindrical pore, a layer of 6-aminouracil molecules was firstly grafted on the nanopore inner wall. Based on the specific molecule-ion bindings, they used (1,1'-(((1,2-diphenylethene-1,2-diyl)bis(4,1phenylene))bis(5-methylpyrimidine-2,4(1H,3H)dione))) (TPE-2D) and $\mathrm{Hg}^{2+}$ as "bricks" to build a 3D macro structure inside the pore (Figure 15 b). The pore size is extremely shrunken, thus a closed state was achieved in the presence of $\mathrm{Hg}^{2+}$. An opposite effect was obtained by $\mathrm{S}^{2-}$ that destroy the macro complexes because of the extreme high binding force between $\mathrm{Hg}^{2+}$ and $\mathrm{S}^{2-}$ due to low solubility of $\mathrm{H}_{\mathrm{g}} \mathrm{S}\left(\mathrm{K}_{\mathrm{sp}}=1.6 \times 10^{-52}(\mathrm{~mol} / \mathrm{kg})^{2}\right)$. The whole procedure was proved by both current and fluorescence measurements. 


\section{WILEY-VCH}

\subsubsection{Ion selective hybrid biological/polymer nanopore}

The lipid bilayer as support of biological nanopores has weak stability facing solution property changes. Confinement of biological pore inside track-etched polymer nanopore can not only improve the stability of supporting stability but also give ion gating properties. This gain of function to the polymer membranes comes from their original ion permeability determined by protein structure. Our group has achieved insertion of Gramicidin-A inside PC membrane pretreated by ethanol[9, 10] and inside PET membrane functionalized by ALD[54]. Both experimental[9] and molecular dynamic simulation[224] results confirmed higher $\mathrm{K}^{+}$ diffusion than other cations such as $\mathrm{Na}^{+}, \mathrm{Ca}^{2+}$ and $\mathrm{Mg}^{2+}$ due to the $\beta$-helical gA conformation kept at least partially in the pore center. The most remarkable result is that such nanopore is totally impermeable to protons[9]. This was explained by a breakage of the water molecule line along the nanopore length[225]. Besides Gramicidin-A, Amphotericin B[7] and nystatin $\mathrm{A} 1[226]$ were also reported being confined in PC track-etched membranes showing a $\mathrm{Ca}^{2+}$ selectivity at different levels thanks to the partially remaining of their original structures in biological condition[227].

\subsubsection{Ion selective hybrid metal organic frameworks/polymer nanopore}

Recently, a new hybrid nanopore combining metal organic frameworks (MOFs) and polymer track-etched nanopore has been designed for ion sieving[228, 229]. Lu et al. synthesized UiO66- $(\mathrm{COOH})_{2}$ crystals at the tip region of a bullet-shaped PET nanopore[228]. Benzoic acid groups were immobilized on pore walls as ligands to synthesize the MOFs and to eliminate any possible voids between MOFs and pore wall. The synthesized UiO-66- $(\mathrm{COOH})_{2}$ crystals exhibited an aperture pore size of about $6 \AA$. This ultra-small pore size and their negative charges make the hybrid pore much more asymmetric presenting a high rectification behavior. The carboxylic acid groups in MOFs in such a small diameter can thus bind divalent cations resulting in a $\mathrm{K}^{+} / \mathrm{Mg}^{2+}, \mathrm{Na}^{+} / \mathrm{Mg}^{2+}$ and $\mathrm{Li}^{+} / \mathrm{Mg}^{2+}$ selectivity higher than 1000 . With the same 
principle, $\mathrm{Li}$ et al. synthesized positively charged $\mathrm{UiO}-66-\mathrm{NH}_{2}$ and $\mathrm{UiO}-66-\mathrm{N}^{+}\left(\mathrm{CH}_{3}\right)_{3}$ inside bullet-like PET nanopore[229]. As the same as divalent cation binding by UiO-66-(COOH $)_{2}$ for monovalent cation selectivity, positively charged MOFs in PET nanopore show an ultrahigh permeability of $\mathrm{F}^{-}$and an $\mathrm{F}^{-} / \mathrm{Cl}^{-}$selectivity of 192 .

\subsection{Molecule sensing}

\subsubsection{Transient state sensing}

Transient state sensing in nanopore technology is based on the resistive pulse[230]. It can be regarded as a nanometer-scale Coulter counter. A single nanopore is placed between two reservoirs filled with electrolyte solutions. A transmembrane electrical bias is applied to drive molecule translocations through the nanopore. Ion currents are recorded as a function of time. When a molecule enters and translocates through the nanopore, a transient current fluctuation can be measured in an optimized condition. This kind of analyzing method has been investigated in several kinds of nanopores including biological nanopore[11], silicon nitride nanopore[27], graphene nanopore[231], $\mathrm{MoS}_{2}$ nanopore[232] and track-etched nanopore[233, 234].

\section{Nanoparticle characterization}

Resistive pulse technique has been combined with cylindrical track-etched nanopores for nanoparticle detection[235]. The signals obtained by translocating nanoparticles through track-etched nanopore can be influenced either by the particle's intrinsic properties such as shape, surface charge, size or by properties of the nanopore where the surface state, pore shape can play an important role[236]. When a charged nanoparticle passes through a pore in an electrolyte solution, usually a current decrease can be observed because of the occupation. The findings of Menestrina $e t$ al. showed a more complex situation for such passage which can modulate local ionic concentrations and cause formation of positive peaks in track-etched nanopore[237]. Polystyrene particles with diameters of several hundreds of nanometers were 


\section{WILEY-VCH}

translocated pores with diameter close to $1000 \mathrm{~nm}$ in $\mathrm{KCl}$ solution. At low concentrations and large ratios of the particle and pore diameters, the amplitude of the current increase becomes even higher than the current decrease at the entrance for charged particles. To explain that, numerical simulations were investigated by solving coupled PNP and NS equations. When particles approach the pore, the particle depletes the region adjacent to the pore entrance with co-ions. The current carried by co-ion decreases as co-ions move in the same direction of the particle. When they exit the pore, an ion enrichment of counterions occurs at the pore-end leading to a current increase.

Resistive pulse amplitude can be decreased by a particle with a high surface charge density. Indeed, the counterions driven by the nanoparticle can cause less difference by replacing the electrolytes by the particle. Such phenomenon was typically reported during the translocation of DNA through SiN nanopore at low salt concentration[238]. Qiu et al. found that highly charged particles can cause a larger current blockage when carboxylated polystyrene particles translocated through PET and PC nanopores in diameter of around $1000 \mathrm{~nm}$ [239]. Both experimental and numerical results showed that in the zone in front of the passing particles, an ion depletion occurs contributing to current blockage. So the current decrease amplitude can be determined by both particle size and the ion depletion zone size. A higher particle surface charge density prompts a more pronounced concentration polarization and a large ion depletion zone resulting in a higher current blockage. As shown above, particle surface charge can influence a lot the signals so that translocating nanoparticles through nanopores. This can be a good tool to determine charges of particles. Other than translocating, the approach of nanoparticles near the pore entrance without translocation can also be a method to learn about particle charge[240].

Besides the particle charge, particle shape can also influence the resistive pulse in a certain environment.[241]. This can be utilized to distinguish objects by shape using pores with longitudinal irregularities. Qiu et al. fabricated a PET track-etched nanopore with undulating 


\section{WILEY-VCH}

pore diameter performing chemical etching of the films in $0.5 \mathrm{M} \mathrm{NaOH}, 70^{\circ} \mathrm{C}$ [242]. Results from passage of spherical particles and rods through large aspect ratio pores with longitudinal irregularities confirmed that the shape of current pulses depends on the shape and length of the particles. It showed that a volume excluded by the rods is larger than its geometrical volume. This undergoes the rod rotations inside the pore. Meanwhile, the pore with modulating diameters enable the rotational freedom of particles leading to different signals between spherical particles and rods.

As the pore structure can have an impact on translocation signals, translocating particles with known shape and size can then reveal pore structure reversely. By translocating carboxylfunctionalized polystyrene particles through PET nanopore, Pevarnik et al. revealed pore structure from signal shapes[243]. As event depths have quantitative relationship with pore diameter and particle size, successive fluctuations in current traces can be explained by longitudinal irregularities of the pore. Thus, by quantitative calculation, a detailed pore structure can be given. Another research from Siwy's group performed particle translocation in a pore where at least one wider zone called a cavity separates two narrower regions of different lengths[50]. Particles entering from the side with the shorter narrow zone will not get focused and will undergo a wide range of radial positions in the first narrow zone especially in the cavity. This cavity effect slows down the particle velocity. When the particles enter the pore from the longer narrow entrance, they reach focused positions, and radial distribution of their trajectories will be narrower. In this case, there are few effects of the cavity on particle movement, so the velocity can be higher and the event duration becomes shorter. Such a direction dependent transit velocity can reflect the existence of the cavity in the pore and its position.

The particles can be a suitable model to understand the colloids transport under confinement. They allow characterizing how the surface state influences their transport. It was reported that the energy barrier of entrance for a negativity charged nanoparticle $(\sim 100 \mathrm{~nm})$ is smaller for a 


\section{WILEY-VCH}

negative charge nanopore than a neutral hydrophobic one with a similar diameter[235]. Such counterintuitive results were also observed for DNA[244].

\section{DNA detection}

One of the most important tasks of nanopore technology is DNA characterization. Biological pores have been used for commercial DNA sequencing[245] and solid-state nanopores are investigated to provide more stable, reliable and accurate applications[246]. As solid-state nanopore, track-etched polymer nanopore are also used for DNA detection[233, 234, 247]. Kaya et al. investigated the effect of the pore geometry on DNA translocation by fabricating conical PET nanopore with different angles using methanol[248]. They showed that higher cone angle with higher electric field promotes the translocation phenomena. By solving PNP equation and performing translocation of DNA molecules in conical nanopores, Dinler et al. showed that variations in surface and DNA charge density cause conductive distortions and DNA charge density became different during translocation than its native form in the solution[249].

Our group improved the DNA detection by functionalizing track-etched nanopore by inserting biological pores[84] or by ALD coating of $\mathrm{Al}_{2} \mathrm{O}_{3}$ layer[49]. Non-modified $\alpha$-hemolysin was inserted in PET cylindrical nanopore of which the pore size is reduced by ALD deposited $\mathrm{ZnO}-\mathrm{Al}_{2} \mathrm{O}_{3}$ and finally coated with trimethyl silane to ensure the hydrophobic environment to the $\alpha$-hemolysin barrel $_{[84]}$. Polynucleotide discrimination is achieved as poly(A), poly(U) and poly $(\mathrm{C})$ show different blockade rate at $2.9 \%, 4.0 \%$ and $5.1 \%$. The fact that $\alpha$-hemolysin kept its structure was confirmed by molecular dynamic simulation[250]. Another work showed that $\mathrm{Al}_{2} \mathrm{O}_{3}$ coating without biological in a conical nanopore can slow down the translocation velocity of DNA strands[49]. Even short DNA having 10 and 40 nucleotides can be detected. In addition the $\mathrm{Al}_{2} \mathrm{O}_{3}$ also allows improving the signal-to-noise ratio of 2 and 4 for ssDNA and dsDNA. 


\section{WILEY-VCH}

\section{Protein sensing}

Followed by DNA characterization, protein characterization using nanopore appeals more and more attention because of the importance of such living molecules[251]. However, it appears much higher difficulty compared to DNA due to its structural complexity. Despite difficulties, resistive pulse measurements based on track-etched nanopore have been also done for protein detection mainly by Martin's group[252]. Sexton et al. functionalized a PET conical nanopore by Au electroless plating and PEG-thiol coordination for BSA and the BSA/anti-BSA-Fab detection[253]. The PEG chains are used for avoiding protein adsorption. The diameter of the tip was designed with a similar size of the proteins to increase signal amplitude and to have specificity for desired analyte detection. Further investigation using similar systems indicates that the measured duration of events is much longer than the time needed for proteins to pass through the sensing zone[254]. This means that proteins undergo adsorption and desorption to/from the pore wall justified by modeling.

The track-etched nanopore for the protein detection presents several advantages. First the track-etched nanopore has a long lifetime and can be used several weeks especially after PEG grafting. The etching performed under aqueous solution allows doing the nanopore opening, functionalization and electrical measurement one pot. In addition, the high aspect ratio provides a long-sensing zone. This involves a dwell time of protein at ms scale. Thus the protein can be detected without using a high-speed amplifier conversely to the SiN[255]. This low aspect ratio is also the main limit of such nanopore since it decreases the resolution. To improve the resolution, the surface functionalization can be optimized using ethylene diamine [256]. Despite this disadvantage, track-etched nanopore show strong ability to discriminate larger objects with a high aspect ratio such as in protein aggregation. Finally, the resistive pulse is not the only way for protein sensing using track-etched nanopore. The current 


\section{WILEY-VCH}

rectification allows the steady state protein sensing with grafted anchored molecules on the pore wall (see section 4.3.2).

\section{Protein aggregation/degradation analyzing}

Compared to other nanopores, track-etched polymer nanopore can have higher noise due to surface charges, polymer chains and the membrane thickness limits the sensitivity to distinguish small structures like nucleic acid base[257]. However, variable geometries especially conical nanopore gives the possibility to analyze large polymers or rigid fiber which cannot be able to be captured by typical biological or solid-state nanopores. This advantage comes from the large base side (some hundreds nanometers) which permit the entrance of large polymers inside the pore and its cone shape which can orient rigid fiber or compress large flexible polymers to the tip side. This last of several nanometers ensures the resistive-pulse detection.

Our group has developed continuous real time analyzing protein aggregation and their degradation based on PEG-functionalized conical track-etched nanopore[258-260]. It has been proved that amyloids are involved in many age-related degenerative diseases including Alzheimer's and Parkinson's by their misfolding and aggregation[261]. Figure 16 a shows the aggregation process of amyloid proteins including four steps among which the intermediates are the most toxic[262]. The analyzing of formation of amyloid intermediates is achieved using PEG functionalized conical nanopore where PEG chains are used as antifouling coatings (Figure 16 b)[258]. By continuous translocating solutions containing initially only monomers, a change in events distributions is observed as shown in figure $16 \mathrm{c}$. The method allows detecting several populations of protein aggregates in the same sample. Figure $16 \mathrm{~d}$ and e show degradation of $\beta$-lactoglobulin amyloid degradation by pepsin (Figure 16 d) and trypsin (Figure 16 e)[260]. It was found that with pepsin, a reaggregation occurs with appearance of larger aggregate after autodegradation of pepsin. Conversely, the 


\section{WILEY-VCH}

digestion by trypsin does not show any reaggregation process. With similar method, Giamblanco et al. also studied heparin-induced tau aggregation[259]. Continuous analyzing of aggregation of wild type tau187 and tau187 with P301L mutation shows different process. The tau187P301L is found more prone to form oligomers compared to tau187WT and a higher structural flexibility. Despite a lower resolution than low aspect ratio nanopore (i.e., SiN nanopore), the track-etched nanopores show promising results to analyze amyloid fibrils. Apart from analyzing protein aggregates in solution as an ensemble experiment, our group also proposed to detect enzymatic degradation of large polymers by measuring individual reactions in conical nanopore[139]. Translocation of large flexible hyaluronic acids in long conical nanopore $(13 \mu \mathrm{m})$ from the base side can be explained by scaling law using blob models. Different signals were observed in dilute and semi-dilute regimes. An asymmetry in the event signal can be attributed to polymer compression (Figure $17 \mathrm{a}$ and $\mathrm{b}$ ). The enzymesubstrate interactions are measured by grafting hyaluronidase at the tip side. Long events of about 1 second are observed related to the enzyme-substrate complexion (Figure $17 \mathrm{c}$ ). Then inhibitors of quercitine were added into systems to prove the specific interaction. With inhibited enzymes, long events can no longer be detected but small pics without complexion (Figure $17 \mathrm{~d}$ ). This offers a possibility to characterize large molecules and individual enzyme-substrate interactions inside nanopore.

\subsubsection{Steady state sensing}

Steady state sensing is based on nanopore modification due to the binding target/host molecules. Typically, the charge and/or effective pore size modulate the ion transport characterized by current rectification and/or the pore conductance. Numerous specific functional moieties were grafted inside nanopore to provide specific interactions with the target molecules. These interactions can be triggered by bioconjugated binding, nucleic-acid hybridization, enzyme-substrate reaction, or small molecule. With the simplicity of surface 
functionalization and the various pore geometries, the track-etched nanopores make a robust platform for steady state sensing.

Sensing by trapping small molecules in bioconjugates

As macromolecules can form molecule cage to trigger ions, the same principle can also be performed for small molecules. Li et al. have investigated immobilized sequence-specific aptamers (SSA) as probes inside a conical nanopore to detect adenosine[263]. Due to the binding effect of the specific nucleotide sequence to adenosine, SSA can form a stable complex. The folded chain around adenosine leads to a pore opening that induces a higher current under $\pm 2 \mathrm{~V}$ than for extended DNA probes. The cocaine was detected by Wang et al. using a bullet-like nanopore functionalized with an aptamer[264] (Figure 18 a). In this work, a bodipy493/503-modified target DNA aptamer was added with cocaine. The latter is inserted into the partially hybrid structure. This sandwich complex of probe-cocaine-aptamer modulates the ion transport with a high current change ratio measured at $+2 \mathrm{~V}$. This nanopore shows suitable selectivity to identify cocaine from other molecules with similar structures such as tropinone, atropine and glucose. The stereoselective detection of amino acids was achieved by Ali et al. based on conical nanopore functionalized with polydopamine (PDP)BSA[265]. Such nanopore is suitable to specifically recognize the L-Trp over D-Trp isomers through specific BSA-tryptophan interactions. The detection can work in a large concentration range from $100 \mathrm{mM}$ to $1.5 \mathrm{mM}$ of L-Trp.

\section{Sensing by molecule-functional group reaction}

The molecule sensing can be based on chemical reactions between functional groups and target molecules. Oxidation is a good example to illustrate that. Ali et al. have grafted boronic ester carbamate (BEC), a reductive molecule, inside a conical nanopore to detect hydrogen peroxides $\left(\mathrm{H}_{2} \mathrm{O}_{2}\right)$ [266] (Figure $18 \mathrm{~b}$ ). Due to the strong oxidation ability of $\mathrm{H}_{2} \mathrm{O}_{2}$, it can break 


\section{WILEY-VCH}

carbamate bonds to expose the amine moieties. The nanopore surface changes from relatively hydrophobic to hydrophilic and positively charged. Another interesting research based on peptides functionalized conical nanopore was done by Xiao et al. [267]. In this paper, the peptide contains CGGC sequences, in which thiol groups can be oxidized to form a disulfide bond. The peptide chain was then forced to fold because of the S-S bond. Therefore, an $\mathrm{O}_{2}$ sensor was achieved sensitive to a concentration at least $0.91 \mathrm{mg} / \mathrm{L}$.

\section{Sensing by nucleic acid hybridization}

The hydrogen bond is the intermolecular interaction involved in the nucleic acid structure and thus the DNA/RNA hybridization. To achieve DNA/RNA sensing, Ali et al. grafted Peptide Nucleic Acid (PNA) on a conical pore surface[268]. When the complementary DNA oligomers are added into the solution, they can undergo a subsequent hybridization with tethered PNA probe. The highly negative charge density of DNA backbones can then change ionic current rectification behaviors. Non-complementary DNA segments and DNA oligomers with base mismatches do not have or have lower affinity with the probe leading to a less change in ICR. With similar principle, Sun et al. developed a DNA probe functionalized biosensor to detect DNA mismatch[269]. As shown in figure $18 \mathrm{c}$, as the complementary strands form more hydrogen bonds, more probes will be hybridized compared to the ones with a mismatch. Due to the high charge density of the back bones of target chains, an enhanced selectivity to cation transport (ion current rectification (ICR) change) will be measured as consequence. It is also confirmed that the number of base mismatches on target has a quantitative relationship with ICR changes. This method was also used by Liao et al. to detect microRNAs using a phosphorodiamidate morpholino oligos (PMO)-functionalized nanochannel biosensor[270]. This biosensor attained a reliable limit of detection down to 1 $\mathrm{fM}$ in PBS and $10 \mathrm{fM}$ in serum sample showing strong application prospects in clinical diagnosis. 


\section{WILEY-VCH}

Sensing by biomolecular recognition

In biology systems, specific biomolecular recognition plays an important role in such as immune response, ligand-mediated cell activation, cell communication and embryogenesis. Biosensors inspired by ligand-gated ion channel have been largely developed using tracketched nanochannels. The general ideal is that one a probe is immobilized on the pore surface and then a target biomolecules with specific recognition can be bounded. The specific binding of target molecules can be shown by the behavior change of ionic transport. Ali's group has reported numerous of systems based on that concept. A streptavidin sensor was prepared by grafting biotin-PEO3-Amine in a conical pore[271]. The strong and specific biotinstreptavidin interaction ensures the accurate detection of streptavidin was characterized by a change of I-V curves that did not happen for lysozyme and BSA. A sensor of concanavalin A (Con A) based on sugar-lectin interactions was achieved by grafting horseradish peroxidase (HRP) in nanochannels of two geometries[272]. Blocking effect in cylindrical pores was observed by a decrease of pore conductance. In addition a change of current rectification was shown in a conical pore because of the modification of surface state due to the specific binding. Another sensor to detect Con A was also achieved using lectin carbohydrate interaction in bullet-like pores by grafting $\mathrm{p}$-aminophenil $\alpha$-D-mannopyranoside (APMP)[142]. The sensor was confirmed by its specificity to bind Con A compared to lysozyme and BSA. By using mannose, the sensor is reusable. Ali et al. designed a biosensor based on aptamer-lysozyme binding in a cylindrical nanopore[43].

A DNA aptamer was immobilized on pore walls by EDC/sulfo-NHS catalyzing. An asymmetric addition of lysozyme can highly rectify current-voltage curves of the pore because of the positive charges in lysozyme and negative charges in DNA aptamer. It showed a very good specificity of lysozyme compared to other proteins including cytochrome C, avidin and bovine hemoglobin. Our group has proposed a reversible PLL-PEG biotin 


\section{WILEY-VCH}

functionalized nanopore. It shows a reliable ability to detect streptavidin with a very quick functionalization procedure[131]. To go further from that, another system of "multi-step biosensing" was also demonstrated by our group on combining biotin-avidin and proteinantibody interactions by successively grafting PEG-biotin, avidin, biotinylated proteins and antibodies[85] (Figure $18 \mathrm{~d}$ ). This complex building shows the possibility to design multiple steps, multiple targets sensing process using biomolecular recognition. Ahlawat et al. used antibody-antigen for designing biosensors in PC membranes[273]. Mesothelin antibody was immobilized in gold-coated PC membranes via thiols and EDC/NHS linking. A rapid decrease in ionic current by adding mesothelin antigen was shown presenting a rapid detection ability of such antigen.

\section{Sensing by macro structure construction/destruction}

Similar to principles reported in the section 4.2 .3 , sensing by macro structure reversible selfassembly can also be also applied for molecules. A macro structure can sterically block the pore to reduce ionic conductance confirming the presence of target molecules. Xia et al. used DNA molecules as bricks to build long DNA chains using DNA linker and one signal probe for ATP sensing[274]. The probe DNA was firstly immobilized in a pore. Then, the signal probed with aptamer sequence and DNA linkers was added to the system. The signal probes and DNA hybrid linkers together to form a long chain which can block the channel by steric effect. When the target molecule was added, a strong binding interaction between aptamers and ATP make the long chain breakup leading to the pore opening. A dense macro structures were designed by Lou et al.[275]. To do so, the authors modified cylindrical nanopore using ((1, 2-diphenylethene-1, 2-diyl) bis (1, 4-phenylene)-1, 1'-diboronic acid (TPEDB)) and glucose in a capture probes (4-aminophenylboronic acid (PBA)) (Figure 18 e). In this PBA functionalized nanopore, if the solution contains glucose, it can be combined with TPEDB under a laser to form a 3D structure. Guo et al proposed another 3D DNA keepers using Y- 


\section{WILEY-VCH}

DNA, ATP aptamer as probe and linker DNA for ATP and DNase I.[276]. Both of them used target molecules as building blocks for constructing signal amplifiers.

\section{Sensing by enzyme-substrate reaction}

A specific biochemical reaction as enzymatic reaction with corresponding substrate was as well investigated for biosensing in track-etched nanopore. Ali and co-workers successfully immobilized horseradish peroxidase (HRP) in a conical pore[277] (Figure 18 f).If there is $\mathrm{H}_{2} \mathrm{O}_{2}$ in the solution, the immobilized HRP enzyme can be activated by $\mathrm{H}_{2} \mathrm{O}_{2}$ turning out to be an oxidative form. The oxidative HRP enzyme can then oxidize substrates of 2, 2'-azinobis (3-ethylbenzothiazoline-6-sulphonic acid) (ABTS). Finally, the resulted ABTS radical cations can decrease the current under $+2 \mathrm{~V}$ because of its positive charges. The sensibility can reach $10 \mathrm{nM}$ of $\mathrm{H}_{2} \mathrm{O}_{2}$. Moreover, a urea sensing was achieved by Pérez-Mitta et al. using physical adsorbed PAH-Urease in bullet-like nanopores[278].

\subsection{Energy}

\subsubsection{Salinity gradient energy conversion}

The osmotic energy is harvested using two methods: the pressure retarded osmosis (PRO)[279, 280] and the reversed electrodialysis (RED)[281]. Even some facilities based on PRO technique are in working[280]; it still remains a lot of improvement. The RED utilizes ionselective membranes (IEMs). When ions diffuse selectively through IEMs driven by salinity gradient, the movements of the ion can generate electron flux by coupling redox electrodes. To get this electric current and transmembrane potential, high ion selectivity is requested to make sure that positive ions go for one direction while negative ions go oppositely. Thus a high charge density and a high charge asymmetry inside pores are required to modulate ion transport properties for high ion selectivity. Recent findings of extremely high osmotic power density using new materials as membrane supports have revived the field[153]. High performance was obtained by a single boron nitride nanotube[282] with a single pore power 


\section{WILEY-VCH}

of $20 \mathrm{pW}$ and molybdenum disulfide 2D nanopore[283] of $500 \mathrm{pW}$. At multipore scale, dissymetrical membranes are also recently investigated[97]. The ion selectivity and membrane resistance are always two limiting factors for this technique.

Biomimetic ion channels can be a suitable approach to improve the membrane for osmotic energy harvesting[284]. Research based on track-etched nanopore/membrane functionalized by polyelectrolytes can be a good candidate because of the low cost, the good chemical, mechanical properties and the high ion selectivity involved by the high charge density of polyelectrolytes. Our group proposed polyelectrolytes functionalization in track-etched nanopores to improve osmotic energy generation[134, 285]. A surface modification by chitosan / poly(acrylic acid) (CH/PAA) self-assembly was achieved by layer-by-layer deposition on the surface of a conical nanopore[134]. Chitosan was chosen as polyelectrolyte because it is not soluble and uncharged at neutral and basic $\mathrm{pH}$. Then the net negative charges of PAA increase surface charge density. Above pH 7.6 with 1000 folds of salinity gradient, a single pore power around $25 \mathrm{pW}$ is obtained which is comparable with boron nitride nanotubes. Despite good performances the conical shape limits pore density for upscale applications. Inspired by electric eels, the highly charged hydrogel shows good potentiality to generate huge voltage from the osmosis phenomena[286]. Based on this, we then proposed hydrogel frameworks inside track-etched nanopores as a 3D functionalization[285]. The high charge density inside ensures the ion selectivity of the pore. Two geometries of conical and cylindrical pores were compared. Experimental results show that with hydrogel functionalization, cylindrical nanopore can have the same performance as conical ones. This makes it possible to use cylindrical nanopore to fabricate multipore membranes. By stacking alternately two membranes of $10^{9}$ pores $/ \mathrm{m}^{2}$ functionalized by a positively charged hydrogel and two membranes of $10^{9}$ pores $/ \mathrm{m}^{2}$ functionalized by a negatively charged hydrogel, a global power density of $0.37 \mathrm{~W} / \mathrm{m}^{2}$ was achieved. Lin et al. improved single pore performance by enlarging pore diameter to $400 \mathrm{~nm}$ for tip and $2 \mu \mathrm{m}$ for the base and filling with poly-L-lysine 


\section{WILEY-VCH}

of high molecular weight (150 kDa to $300 \mathrm{kDa})$. The large mesopore increases ionic conductance reaching $\mu \mathrm{S}$. Thus, single pore power is roughly estimated to be $120 \mathrm{pW}$. Recent findings focused on the impact of track-etched nanopore geometries on osmotic energy conversion. Hsu et al. designed a funnel shape device based on PET membrane[287]. For a pore with tip diameter of $15 \mathrm{~nm}$, the osmotic current and the reversed voltage under 500 $\mathrm{mM} / 1 \mathrm{mM} \mathrm{KCl}$ gradient at $\mathrm{pH} 10$ were measured as $19.1 \mathrm{pA}$ and $130 \mathrm{mV}$ meaning a single pore power of $2.5 \mathrm{pW}$. By changing surface charge density, an interesting phenomenon was found that a very high charge density can decrease the power output. This can be explained that ion concentration polarization can be highly enhanced in a channel with high charge density. This concentration polarization then decreases in the effective salinity ratio in the channel. Laucirica et al. developed another asymmetric track-etched nanopore based on bullet shape for osmotic energy[288]. Geometrical parameters and solution conditions were optimized that a maximum single pore power of $80 \mathrm{pW}$ was achieved in such PET nanopore. This high single pore power from high ion selectivity exceeded the conventional cylindrical and conical nanopores showing the abundant possibilities of track-etched nanopore in this topic.

The advancement in single nanopore can be exciting and inspiring. However, a large barrier can exist between estimated power density based on data of single nanopore and measured power density on multipore membrane. This can be found by experiments from single pore to multipore membrane to stacked membranes for a high pore density[285]. It has been reported that a pore density of $10^{7}$ pores $/ \mathrm{cm}^{2}$ can be critical for cylindrical pores of $22 \mathrm{~nm}$ in diameter[289]. This comes from the changes of ion concentration distribution and salinity gradient influenced by the neighboring pores. In porous membrane systems, the transport is governed by a constant entering resistance[290].

As ion selectivity of track-etched nanopores comes from the asymmetry, it can be improved by subjoining second membrane to increase asymmetries for a higher ion selectivity. Zhang et 


\section{WILEY-VCH}

al. developed a heterogeneous membrane to perform as ion pumps[291]. A highly positively charged block copolymer (BCP) as polystyrene-b-poly(4-vinyl pyridine) $\left(\mathrm{PS}_{48400}\right.$-b$\left.\mathrm{P}_{4} \mathrm{VP}_{21300}\right)$ is coated on the tip side of conical membrane as shown in figure 19 a. The success of this coating and the introduction of related high charge density are confirmed by an increase of pore conductance at low electrolyte concentration. As the track-etched nanopore wall is covered by carboxylic acid groups which have negative charges at $\mathrm{pH}$. The positively charged BCP layer and negatively charged track-etched pore wall form an ionic diode with ion rectification ratio as high as 1075 highly controlled by $\mathrm{pH}$. A maximum power density of $0.35 \mathrm{~W} \mathrm{~m}^{-2}$ was reached using 0.5 and $0.01 \mathrm{M} \mathrm{NaCl}$ solutions. Then the same group designed a double gated hybrid membrane by coating a layer of BCP (PS-b-P4VP) on the base side of conical nanopore and grafting PAA at the tip side[292] (Figure 19 b). Thus, a $\mathrm{pH}$-gated ion pump is ready. By changing $\mathrm{pH}$ of two reservoirs separately, the pump shows different behaviors as alternating gate ion pumps, pump-channel or fail-safe ion pumps. The pore is highly selective to anions that can unidirectionally pump them. Well, it can be a good candidate for osmotic energy harvesting or desalination.

\subsubsection{Photoelectric conversion}

Photoelectric is one of the most important sustainable energy sources as is directly taken advantage of energy from sun light[293]. By now photoelectricity is generated by large facilities based on inorganic and organic materials where silicon derives take majority[294]. By learning from proton pump of biological systems, photoelectricity has been also investigated using track-etched nanopores by constructing photo-driven ion pumps. Wen et al. established a photoelectric conversion system based on i-motif DNA functionalized conical nanopore[295]. C4 DNA molecules were immobilized inside conical PET nanopores by EDC chemistry (Figure 20a). The negative charges of DNA molecules ensure the transport of protons across the membranes. Such functionalized nanopore was 


\section{WILEY-VCH}

immersed in 8-hydroxypyrene-1,3,6-trisulfonate (HA) solution used as the photo-acid molecule. The HA molecule can release a proton when it is irradiated by light. This process is reversible, ionized HA can receive protons in darkness. Based on that, when the system undergoes light irradiation, the released protons diffuse across the cation selective nanopore. Then with the help of electrodes, a current can be obtained directly. A similar system was also achieved combining PET conical membranes and photosystem II (PSII) complex is a kind of natural photoelectric conversion material extracted from green plants and some certain bacteria (Figure 20b)[296]. This series of research give a new insight in designing new energy conversion devices combining other light-responsive compounds[297].

\subsubsection{Thermoelectric conversion}

Another possibility of an energy source comes from a temperature gradient known as thermoelectricity[298]. Based on Seebeck effect, different temperatures can form different free electron density in metal and different carrier density in the semi-conductor. To get equilibrium between two regions, the diffusion of electrons or carriers will occur to generate an electric current. The similar principle can be applied in nanofluidic for energy conversion from thermo-osmotic flows[299]. Thus, it is also possible to generate direct ionic flow between two reservoirs in different temperatures connected by an ion-selective membrane. Based on this, Xie et al. investigated the low- grade heat energy harvesting using a conical track-etched PI nanopore[300]. With $\mathrm{KCl}$ solution of $1 \mathrm{M}$ in both sides of the nanopore, a temperature difference of $40{ }^{\circ} \mathrm{C}\left(\mathrm{T}_{\mathrm{h}}=65^{\circ} \mathrm{C}, \mathrm{T}_{\mathrm{c}}=25^{\circ} \mathrm{C}\right)$ between two solutions can generate a current of $1.3 \mathrm{nA}$ with $\mathrm{D}_{\text {tip }}=11.27 \mathrm{~nm}$. The electrolyte concentration of $1 \mathrm{M}$ and high temperature at the base side has been confirmed as optimal conditions for thermoelectric power generation in such device. Therefore a single pore power of more $20 \mathrm{pW}$ was achieved with a temperature gradient of $40^{\circ} \mathrm{C}$ leading to an estimated membrane power density of 88.8 $\mathrm{W} \mathrm{m}{ }^{-2}$. This can be interesting for waste heat energy harvesting, potable self-powered devices 


\section{WILEY-VCH}

and temperature sensing.

Chen et al. explored utilization of energy by combining both salinity and temperature gradients[301]. To do so, the authors constructed a hybrid asymmetric membrane of ultrasmall silica nanochannels (SNCs)/PET track-etched membrane. SNCs with pore size of $2.3 \mathrm{~nm}$ were adhered on the tip side of PET membrane $\left(D_{\text {base }}=570-800 \mathrm{~nm}, \mathrm{D}_{\text {tip }}=12-20 \mathrm{~nm}\right)$. Under $\mathrm{NaCl}$ salinity gradient of 50 folds, a maximum value of power density of $1.0 \mathrm{~W} \mathrm{~m}^{-2}$ and current density of $34.7 \mathrm{~A} \mathrm{~m}^{-2}$ is obtained. Then, a supplementary temperature gradient of $10 \mathrm{~K}$ was applied on the system increasing to power output from 3.27 to $4.59 \mu \mathrm{W}$. The open circuit potential of the hybrid SNC/PET membrane was recorded over ten days as a stable value indicating the possibility of osmotic energy storage.

\section{Conclusion}

This review has summarized the fabrication, functionalization, transport property and applications of track-etched nanopore. During the past 20 years, there was a continuous effort to growth and development in the science and technology of track-etched nanopore. The thrust of the advances has involved the improvement of our understanding of the physics of nanofluidic devices as well as an increased mastery in the design, construction and functionalization of polymeric nanopores of different dimensions. Consequently, to these efforts, track-etched nanopore has become an important platform for numerous applications such as ionic diodes, molecule sensing and energy harvesting.

Despite the great advances that have been made, it always remains more unknown than what is already clear. One issue is that the control size especially for the conical nanopore is performed after opening. This makes difficult to target an exact nanopore diameter. In other words, there are no methods to open as desired conical nanopore with high precision. However, for the cylindrical nanopore the diameter is more predictable according to the chemical etching conditions. In addition, only electrical current can characterize the functionalization of a single nanopore. The lack of other direct characterizing techniques 


\section{WILEY-VCH}

inside nanopore still bothers researchers. Otherwise, polymer insertion using "grafting to" methods still suffers from the unclear grafting density and its reliable controlling. Polymer insertion using "grafting from" methods has to accomplish the great difference of polymerization between surface growth and growth in confined space. As for molecule sensing, lots of problems remain to be solved for quantitative sensing such as reproducibility of nanopore fabrication and non-specific adsorption of nanopores. In osmotic energy conversion, it seems that a bottleneck of high membrane resistance and ion selectivity limits the power density in track-etched membranes.

Beside these issues, the track-etched nanopores are still not fully exploited. Their functionalization is quite easy and can be controlled even if the latter has not atomically precision of biological nanopore. One of the main advantages of track-etched nanopore is their long lifetime. Compared to the other solid-state nanopore, they are not degraded during the experiment and their structure keep their integrity. Typically, they can be used for several weeks with and without functionalization. Their asymmetrical shape induces the current rectification. This could be used to characterize the kinetics of chemical reaction, polyelectrolytes adsorption or enzymatic reaction under confinement that was not currently investigated. The detection of amyloid was also a new opportunity to explore since it could allow following the kinetic of amyloid growth with information about their structure under continuous measurement. Last but not least, the possibility to bind protein inside such nanopore could permit characterizing in real time their folding/unfolding.

Whatever the application, interdisciplinary cooperation is an effective key. Combining with advanced organic synthesis, surface characterizing techniques, molecular biology, single molecule manipulation techniques such as optical tweezers, magnetic tweezers, other types of nanopores, membranes, supercomputers, cryoelectron microscopy can give abundant possibilities to track-etched nanopores. These allow us to pursue new horizons treating ongoing health, energy, and environmental issues. 


\section{WILEY-VCH}

\section{References}

[1] a) M.J. Ackerman, D.E. Clapham. Ion channels--basic science and clinical disease. The New England journal of medicine, 1997, 336, 1575; b) E. Gouaux, R. Mackinnon. Principles of selective ion transport in channels and pumps. Science (New York, N.Y.), 2005, 310, 1461;

[2] A.L. HODGKIN, A.F. HUXLEY. A quantitative description of membrane current and its application to conduction and excitation in nerve. The Journal of physiology, 1952, 117, 500.

[3] B. Katz, R. Miledi. The statistical nature of the acetycholine potential and its molecular components. The Journal of physiology, 1972, 224, 665.

[4] B. Sakmann, E. Neher. Patch clamp techniques for studying ionic channels in excitable membranes. Annual review of physiology, 1984, 46, 455.

[5] a) D.A. Doyle, A. Lee, J. Lewis, E. Kim, M. Sheng, R. Mackinnon. Crystal Structures of a Complexed and Peptide-Free Membrane Protein-Binding Domain: Molecular Basis of Peptide Recognition by PDZ. Cell, 1996, 85, 1067; b) D.A. Doyle, J. Morais Cabral, R.A. Pfuetzner, A. Kuo, J.M. Gulbis, S.L. Cohen, B.T. Chait, R. MacKinnon. The structure of the potassium channel: Molecular basis of $\mathrm{K}+$ conduction and selectivity. Science (New York, N.Y.), 1998, 280, 69;

[6] R. Mackinnon. Potassium channels and the atomic basis of selective ion conduction (Nobel Lecture). Angewandte Chemie (International ed. in English), 2004, 43, 4265.

[7] D. Thiele, S. Kraszewski, S. Balme, F. Picaud, J.-M. Janot, P. Déjardin. Structure and ionic selectivity of a hybrid polyene/artificial polymer solid state membrane. Soft matter, 2013, 9, 684.

[8] a) M. Barboiu, A. Gilles. From natural to bioassisted and biomimetic artificial water channel systems. Accounts of chemical research, 2013, 46, 2814; b) H. Wang, T.-S. Chung, Y.W. Tong, K. Jeyaseelan, A. Armugam, Z. Chen, M. Hong, W. Meier. Highly permeable and selective pore-spanning biomimetic membrane embedded with aquaporin Z. Small (Weinheim an der Bergstrasse, Germany), 2012, 8, 1185-90, 1125; c) C.Y. Tang, Y. Zhao, R. Wang, C. Hélix-Nielsen, A.G. Fane. Desalination by biomimetic aquaporin membranes: Review of status and prospects. Desalination, 2013, 308, 34;

[9] S. Balme, F. Picaud, S. Kraszewski, P. Déjardin, J.M. Janot, M. Lepoitevin, J. Capomanes, C. Ramseyer, F. Henn. Controlling potassium selectivity and proton blocking in a hybrid biological/solid-state polymer nanoporous membrane. Nanoscale, 2013, 5, 3961.

[10] S. Balme, J.-M. Janot, L. Berardo, F. Henn, D. Bonhenry, S. Kraszewski, F. Picaud, C. Ramseyer. New bioinspired membrane made of a biological ion channel confined into the cylindrical nanopore of a solid-state polymer. Nano Lett., 2011, 11, 712.

[11] J.J. Kasianowicz, E. Brandin, D. Branton, D.W. Deamer. Characterization of individual polynucleotide molecules using a membrane channel. Proceedings of the National Academy of Sciences of the United States of America, 1996, 93, 13770.

[12] a) M. Pastoriza-Gallego, L. Rabah, G. Gibrat, B. Thiebot, F.G. van der Goot, L. Auvray, J.-M. Betton, J. Pelta. Dynamics of unfolded protein transport through an aerolysin pore. J. Am. Chem. Soc., 2011, 133, 2923; b) B. Cressiot, H. Ouldali, M. Pastoriza-Gallego, L. Bacri, F.G. van der Goot, J. Pelta. Aerolysin, a Powerful Protein Sensor for Fundamental Studies and Development of Upcoming Applications. ACS sensors, 2019, 4, 530; c) F. Piguet, H. Ouldali, M. Pastoriza-Gallego, P. Manivet, J. Pelta, A. Oukhaled. Identification of single amino acid differences in uniformly charged homopolymeric peptides with aerolysin nanopore. Nature communications, 2018, 9, 966; d) C. Cao, Y.-L. Ying, Z.-L. Hu, D.-F. Liao, H. Tian, Y.-T. Long. Discrimination of oligonucleotides of different lengths with a wild-type aerolysin nanopore. Nature nanotechnology, 2016, 11, 713; 


\section{WILEY-VCH}

[13] a) I.M. Derrington, T.Z. Butler, M.D. Collins, E. Manrao, M. Pavlenok, M. Niederweis, J.H. Gundlach. Nanopore DNA sequencing with MspA. Proceedings of the National Academy of Sciences of the United States of America, 2010, 107, 16060; b) T.Z. Butler, M. Pavlenok, I.M. Derrington, M. Niederweis, J.H. Gundlach. Single-molecule DNA detection with an engineered MspA protein nanopore. Proceedings of the National Academy of Sciences of the United States of America, 2008, 105, 20647;

[14] O. Yildiz, K.R. Vinothkumar, P. Goswami, W. Kühlbrandt. Structure of the monomeric outer-membrane porin OmpG in the open and closed conformation. The EMBO journal, 2006, 25, 3702.

[15] C. Wloka, N.L. Mutter, M. Soskine, G. Maglia. Alpha-Helical Fragaceatoxin C Nanopore Engineered for Double-Stranded and Single-Stranded Nucleic Acid Analysis. Angewandte Chemie (International ed. in English), 2016, 55, 12494.

[16] M. Soskine, A. Biesemans, B. Moeyaert, S. Cheley, H. Bayley, G. Maglia. An engineered ClyA nanopore detects folded target proteins by selective external association and pore entry. Nano Lett., 2012, 12, 4895.

[17] C.G. Brown, J. Clarke. Nanopore development at Oxford Nanopore. Nature biotechnology, 2016, 34, 810.

[18] E.A. Manrao, I.M. Derrington, A.H. Laszlo, K.W. Langford, M.K. Hopper, N. Gillgren, M. Pavlenok, M. Niederweis, J.H. Gundlach. Reading DNA at single-nucleotide resolution with a mutant MspA nanopore and phi29 DNA polymerase. Nature biotechnology, 2012, 30, 349.

[19] O.S. Smart, J.M. Goodfellow, B.A. Wallace. The pore dimensions of gramicidin A. Biophysical Journal, 1993, 65, 2455.

[20] R.J. Collier, J.A.T. Young. Anthrax toxin. Annual review of cell and developmental biology, 2003, 19, 45.

[21] H. Shogomori, T. Kobayashi. Lysenin: A sphingomyelin specific pore-forming toxin. Biochimica et biophysica acta, 2008, 1780, 612.

[22] B. Cressiot, S.J. Greive, M. Mojtabavi, A.A. Antson, M. Wanunu. Thermostable virus portal proteins as reprogrammable adapters for solid-state nanopore sensors. Nature communications, 2018, 9, 4652.

[23] a) D. Deamer, M. Akeson, D. Branton. Three decades of nanopore sequencing. Nature biotechnology, 2016, 34, 518; b) J. Clarke, H.-C. Wu, L. Jayasinghe, A. Patel, S. Reid, H. Bayley. Continuous base identification for single-molecule nanopore DNA sequencing. Nature nanotechnology, 2009, 4, 265;

[24] a) D. Kim, J.-Y. Lee, J.-S. Yang, J.W. Kim, V.N. Kim, H. Chang. The architecture of SARS-CoV-2 transcriptome, 2020; b) A.D. Davidson, M.K. Williamson, S. Lewis, D.

Shoemark, M.W. Carroll, K. Heesom, M. Zambon, J. Ellis, P.A. Lewis, J.A. Hiscox, D.A. Matthews. Characterisation of the transcriptome and proteome of SARS-CoV-2 using direct RNA sequencing and tandem mass spectrometry reveals evidence for a cell passage induced in-frame deletion in the spike glycoprotein that removes the furin-like cleavage site, 2020;

[25] a) L. Restrepo-Pérez, C. Joo, C. Dekker. Paving the way to single-molecule protein sequencing. Nature nanotechnology, 2018, 13, 786; b) M. Chinappi, F. Cecconi. Protein sequencing via nanopore based devices: A nanofluidics perspective. Journal of physics. Condensed matter : an Institute of Physics journal, 2018, 30, 204002; c) H. Ouldali, K. Sarthak, T. Ensslen, F. Piguet, P. Manivet, J. Pelta, J.C. Behrends, A. Aksimentiev, A. Oukhaled. Electrical recognition of the twenty proteinogenic amino acids using an aerolysin nanopore. Nature biotechnology, 2020, 38, 176; d) T.C. Sutherland, Y.-T. Long, R.-I. Stefureac, I. Bediako-Amoa, H.-B. Kraatz, J.S. Lee. Structure of Peptides Investigated by Nanopore Analysis. Nano Lett., 2004, 4, 1273; e) L. Movileanu, J.P. Schmittschmitt, J.M. Scholtz, H. Bayley. Interactions of peptides with a protein pore. Biophysical Journal, 2005, 89, 1030; 


\section{WILEY-VCH}

[26] C. Dekker. Solid-state nanopores. Nature nanotechnology, 2007, 2, 209.

[27] J. Li, D. Stein, C. McMullan, D. Branton, M.J. Aziz, J.A. Golovchenko. Ion-beam sculpting at nanometre length scales. Nature, 2001, 412, 166.

[28] a) A.J. Storm, J.H. Chen, X.S. Ling, H.W. Zandbergen, C. Dekker. Fabrication of solid-state nanopores with single-nanometre precision. Nature materials, 2003, 2, 537; b) A.J. Storm, J.H. Chen, X.S. Ling, H.W. Zandbergen, C. Dekker. Electron-beam-induced deformations of $\mathrm{SiO} 2$ nanostructures. Journal of Applied Physics, 2005, 98, 14307;

[29] K. Briggs, H. Kwok, V. Tabard-Cossa. Automated fabrication of 2-nm solid-state nanopores for nucleic acid analysis. Small (Weinheim an der Bergstrasse, Germany), 2014, 10, 2077.

[30] K. Yazda, S. Tahir, T. Michel, B. Loubet, M. Manghi, J. Bentin, F. Picaud, J. Palmeri, F. Henn, V. Jourdain. Voltage-activated transport of ions through single-walled carbon nanotubes. Nanoscale, 2017, 9, 11976.

[31] R.H. Baughman, A.A. Zakhidov, W.A. Heer. Carbon nanotubes--the route toward applications. Science (New York, N.Y.), 2002, 297, 787.

[32] a) S. Garaj, S. Liu, J.A. Golovchenko, D. Branton. Molecule-hugging graphene nanopores. Proceedings of the National Academy of Sciences of the United States of America, 2013, 110, 12192; b) G.F. Schneider, S.W. Kowalczyk, V.E. Calado, G. Pandraud, H.W. Zandbergen, L.M.K. Vandersypen, C. Dekker. DNA translocation through graphene nanopores. Nano Lett., 2010, 10, 3163;

[33] S. Liu, B. Lu, Q. Zhao, J. Li, T. Gao, Y. Chen, Y. Zhang, Z. Liu, Z. Fan, F. Yang, L. You, D. Yu. Boron nitride nanopores: Highly sensitive DNA single-molecule detectors. Advanced materials (Deerfield Beach, Fla.), 2013, 25, 4549.

[34] M. Graf, M. Lihter, M. Thakur, V. Georgiou, J. Topolancik, B.R. Ilic, K. Liu, J. Feng, Y. Astier, A. Radenovic. Fabrication and practical applications of molybdenum disulfide nanopores. Nature protocols, 2019, 14, 1130.

[35] M.D. Fischbein, M. Drndić. Electron beam nanosculpting of suspended graphene sheets. Appl. Phys. Lett., 2008, 93, 113107.

[36] a) X. Xu, C. Li, Y. Zhou, Y. Jin. Controllable Shrinking of Glass Capillary Nanopores Down to sub-10 nm by Wet-Chemical Silanization for Signal-Enhanced DNA Translocation. ACS sensors, 2017, 2, 1452; b) R.-J. Yu, Y.-L. Ying, R. Gao, Y.-T. Long. Confined Nanopipette Sensing: From Single Molecules, Single Nanoparticles, to Single Cells. Angewandte Chemie (International ed. in English), 2019, 58, 3706;

[37] P.Y. Apel, Y.E. Korchev, Z. Siwy, R. Spohr, M. Yoshida. Diode-like single-ion track membrane prepared by electro-stopping. Nuclear Instruments and Methods in Physics Research Section B: Beam Interactions with Materials and Atoms, 2001, 184, 337.

[38] a) M.J. Hope, M.B. Bally, G. Webb, P.R. Cullis. Production of large unilamellar vesicles by a rapid extrusion procedure. Characterization of size distribution, trapped volume and ability to maintain a membrane potential. Biochimica et Biophysica Acta (BBA) Biomembranes, 1985, 812, 55; b) P.J. Patty, B.J. Frisken. The Pressure-Dependence of the Size of Extruded Vesicles. Biophysical Journal, 2003, 85, 996;

[39] P.Y. Apel. Fabrication of functional micro- and nanoporous materials from polymers modified by swift heavy ions. Radiation Physics and Chemistry, 2019, 159, 25.

[40] Y. Zhao, J.-M. Janot, E. Balanzat, S. Balme. Mimicking pH-Gated Ionic Channels by Polyelectrolyte Complex Confinement Inside a Single Nanopore. Langmuir : the ACS journal of surfaces and colloids, 2017, 33, 3484.

[41] T. Ma, P. Gaigalas, M. Lepoitevin, I. Plikusiene, M. Bechelany, J.-M. Janot, E. Balanzat, S. Balme. Impact of Polyelectrolyte Multilayers on the Ionic Current Rectification of Conical Nanopores. Langmuir : the ACS journal of surfaces and colloids, 2018, 34, 3405. [42] P. Déjardin, E.N. Vasina, V.V. Berezkin, V.D. Sobolev, V.I. Volkov. Streaming potential in cylindrical pores of poly(ethylene terephthalate) track-etched membranes: 


\section{WILEY-VCH}

Variation of apparent zeta potential with pore radius. Langmuir : the ACS journal of surfaces and colloids, 2005, 21, 4680.

[43] M. Ali, S. Nasir, W. Ensinger. Bioconjugation-induced ionic current rectification in aptamer-modified single cylindrical nanopores. Chemical communications (Cambridge, England), 2015, 51, 3454.

[44] K. Xiao, G. Xie, Z. Zhang, X.-Y. Kong, Q. Liu, P. Li, L. Wen, L. Jiang. Enhanced Stability and Controllability of an Ionic Diode Based on Funnel-Shaped Nanochannels with an Extended Critical Region. Advanced materials (Deerfield Beach, Fla.), 2016, 28, 3345.

[45] J. Cervera, P. Ramirez, S. Mafe, P. Stroeve. Asymmetric nanopore rectification for ion pumping, electrical power generation, and information processing applications.

Electrochimica Acta, 2011, 56, 4504.

[46] a) H. Zhang, Y. Tian, J. Hou, X. Hou, G. Hou, R. Ou, H. Wang, L. Jiang. Bioinspired Smart Gate-Location-Controllable Single Nanochannels: Experiment and Theoretical Simulation. ACS nano, 2015, 9, 12264; b) H. Zhang, X. Hou, Z. Yang, D. Yan, L. Li, Y. Tian, $\mathrm{H}$. Wang, L. Jiang. Bio-inspired smart single asymmetric hourglass nanochannels for continuous shape and ion transport control. Small (Weinheim an der Bergstrasse, Germany), 2015, 11, 786;

[47] a) K. Xiao, L. Chen, Z. Zhang, G. Xie, P. Li, X.-Y. Kong, L. Wen, L. Jiang. A Tunable Ionic Diode Based on a Biomimetic Structure-Tailorable Nanochannel. Angewandte Chemie (International ed. in English), 2017, 56, 8168; b) K. Xiao, L. Chen, G. Xie, P. Li, X.Y. Kong, L. Wen, L. Jiang. A bio-inspired dumbbell-shaped nanochannel with a controllable structure and ionic rectification. Nanoscale, 2018, 10, 6850;

[48] T. Gamble, K. Decker, T.S. Plett, M. Pevarnik, J.-F. Pietschmann, I. Vlassiouk, A. Aksimentiev, Z.S. Siwy. Rectification of Ion Current in Nanopores Depends on the Type of Monovalent Cations: Experiments and Modeling. J. Phys. Chem. C, 2014, 118, 9809.

[49] V. Thangaraj, M. Lepoitevin, M. Smietana, E. Balanzat, M. Bechelany, J.-M. Janot, J.J. Vasseur, S. Subramanian, S. Balme. Detection of short ssDNA and dsDNA by currentvoltage measurements using conical nanopores coated with $\mathrm{A} 12 \mathrm{O} 3$ by atomic layer deposition. Microchim Acta, 2016, 183, 1011.

[50] Y. Qiu, I. Vlassiouk, P. Hinkle, M.E. Toimil-Molares, A.J. Levine, Z.S. Siwy. Role of Particle Focusing in Resistive-Pulse Technique: Direction-Dependent Velocity in Micropores. ACS nano, 2016, 10, 3509.

[51] J. Cervera, B. Schiedt, R. Neumann, S. Mafé, P. Ramírez. Ionic conduction, rectification, and selectivity in single conical nanopores. The Journal of chemical physics, 2006, 124, 104706.

[52] D. Constantin, Z.S. Siwy. Poisson-Nernst-Planck model of ion current rectification through a nanofluidic diode. Physical review. E, Statistical, nonlinear, and soft matter physics, 2007, 76, 41202.

[53] F. Picaud, G. Paris, T. Gharbi, S. Balme, M. Lepoitevin, V. Tangaraj, M. Bechelany, J.M. Janot, E. Balanzat, F. Henn. Biomimetic solution against dewetting in a highly hydrophobic nanopore. Soft matter, 2016, 12, 4903.

[54] A. Abou Chaaya, M. Le Poitevin, S. Cabello-Aguilar, S. Balme, M. Bechelany, S. Kraszewski, F. Picaud, J. Cambedouzou, E. Balanzat, J.-M. Janot, T. Thami, P. Miele, P. Dejardin. Enhanced Ionic Transport Mechanism by Gramicidin A Confined Inside Nanopores Tuned by Atomic Layer Deposition. J. Phys. Chem. C, 2013, 117, 15306.

[55] M. Lepoitevin, T. Ma, M. Bechelany, J.-M. Janot, S. Balme. Functionalization of single solid state nanopores to mimic biological ion channels: A review. Advances in colloid and interface science, 2017, 250, 195.

[56] R.L. Fleischer, P.B. Price, E.M. Symes. Novel Filter for Biological Materials. Science (New York, N.Y.), 1964, 143, 249. 


\section{WILEY-VCH}

[57] H.B. Lück, H. Matthes, B. Gemende, B. Heinrich, W. Pfestorf, W. Seidel, S. Turuc. Production of particle-track membranes by means of a $5 \mathrm{MV}$ tandem accelerator. Nuclear Instruments and Methods in Physics Research Section B: Beam Interactions with Materials and Atoms, 1990, 50, 395.

[58] G.G. Eichholz, J.W. Poston. Principles of Nuclear Radiation Detection, 1985.

[59] E.M. Bringa, R.E. Johnson. Coulomb explosion and thermal spikes. Phys. Rev. Lett., 2002, 88, 165501.

[60] T.W. Cornelius, P.Y. Apel, B. Schiedt, C. Trautmann, M.E. Toimil-Molares, S. Karim, R. Neumann. Investigation of nanopore evolution in ion track-etched polycarbonate membranes. Nuclear Instruments and Methods in Physics Research Section B: Beam Interactions with Materials and Atoms, 2007, 265, 553.

[61] A.I. Vilensky, O.G. Larionov, R.V. Gainutdinov, A.L. Tolstikhina, V.Y. Kabanov, D.L. Zagorski, E.V. Khataibe, A.N. Netchaev, B.V. Mchedlishvili. The heavy ion tracks in polymers investigation by means of high-effective liquid chromatography and atomic-force microscopy. Radiation Measurements, 2001, 34, 75.

[62] P. Apel, A. Schulz, R. Spohr, C. Trautmann, V. Vutsadakis. Tracks of very heavy ions in polymers. Nuclear Instruments and Methods in Physics Research Section B: Beam Interactions with Materials and Atoms, 1997, 131, 55.

[63] P. Apel, A. Schulz, R. Spohr, C. Trautmann, V. Vutsadakis. Track size and track structure in polymer irradiated by heavy ions. Nuclear Instruments and Methods in Physics Research Section B: Beam Interactions with Materials and Atoms, 1998, 146, 468.

[64] P. Apel. Track etching technique in membrane technology. Radiation Measurements, 2001, 34, 559.

[65] Z. Siwy, D. Dobrev, R. Neumann, C. Trautmann, K. Voss. Electro-responsive asymmetric nanopores in polyimide with stable ion-current signal. Applied Physics A: Materials Science \& Processing, 2003, 76, 781.

[66] H. Bessbousse, I. Nandhakumar, M. Decker, M. Barsbay, O. Cuscito, D. Lairez, M.-C. Clochard, T.L. Wade. Functionalized nanoporous track-etched $\beta$-PVDF membrane electrodes for lead(ii) determination by square wave anodic stripping voltammetry. Anal. Methods, 2011, 3, 1351 .

[67] L.I. Kravets, S.N. Dmitriev, P.Y. Apel. The properties and porous structure of polypropylene track membranes. Radiation Measurements, 1995, 25, 729.

[68] a) Y.P. Patil, M.D. Kumbhalkar, S. Jadhav. Extrusion of electroformed giant unilamellar vesicles through track-etched membranes. Chemistry and physics of lipids, 2012, 165, 475; b) S.Y. An, M.-P.N. Bui, Y.J. Nam, K.N. Han, C.A. Li, J. Choo, E.K. Lee, S. Katoh, Y. Kumada, G.H. Seong. Preparation of monodisperse and size-controlled poly(ethylene glycol) hydrogel nanoparticles using liposome templates. Journal of colloid and interface science, 2009, 331, 98;

[69] C. Trautmann, W. Brüchle, R. Spohr, J. Vetter, N. Angert. Pore geometry of etched ion tracks in polyimide. Nuclear Instruments and Methods in Physics Research Section B: Beam Interactions with Materials and Atoms, 1996, 111, 70.

[70] O. Cuscito, M.-C. Clochard, S. Esnouf, N. Betz, D. Lairez. Nanoporous $\beta$-PVDF membranes with selectively functionalized pores. Nuclear Instruments and Methods in Physics Research Section B: Beam Interactions with Materials and Atoms, 2007, 265, 309. [71] L.I. Kravets, S.N. Dmitriev, P.Y. Apel. Production and Properties of Polypropylene Track Membranes. Collect. Czech. Chem. Commun., 1997, 62, 752.

[72] a) P. Jin, H. Mukaibo, L.P. Horne, G.W. Bishop, C.R. Martin. Electroosmotic flow rectification in pyramidal-pore mica membranes. J. Am. Chem. Soc., 2010, 132, 2118; b) Y. Jiang, J. Gao, W. Guo, L. Jiang. Mechanical exfoliation of track-etched two-dimensional layered materials for the fabrication of ultrathin nanopores. Chemical communications (Cambridge, England), 2014, 50, 14149; 


\section{WILEY-VCH}

[73] a) H. Yao, J. Zeng, P. Zhai, Z. Li, Y. Cheng, J. Liu, D. Mo, J. Duan, L. Wang, Y. Sun, J. Liu. Large Rectification Effect of Single Graphene Nanopore Supported by PET Membrane. ACS applied materials \& interfaces, 2017, 9, 11000; b) S.C. O'Hern, M.S.H. Boutilier, J.-C. Idrobo, Y. Song, J. Kong, T. Laoui, M. Atieh, R. Karnik. Selective ionic transport through tunable subnanometer pores in single-layer graphene membranes. Nano Lett., 2014, 14, 1234; [74] Y. Dong, Y. Cheng, G. Xu, H. Cheng, K. Huang, J. Duan, D. Mo, J. Zeng, J. Bai, Y. Sun, J. Liu, H. Yao. Selectively Enhanced Ion Transport in Graphene Oxide Membrane/PET Conical Nanopore System. ACS applied materials \& interfaces, 2019, 11, 14960.

[75] X. Hou, H. Dong, D. Zhu, L. Jiang. Fabrication of stable single nanochannels with controllable ionic rectification. Small (Weinheim an der Bergstrasse, Germany), 2010, 6, 361.

[76] Y. Tian, X. Hou, L. Jiang. Biomimetic ionic rectifier systems: Asymmetric modification of single nanochannels by ion sputtering technology. Journal of

Electroanalytical Chemistry, 2011, 656, 231.

[77] E.B. Kalman, O. Sudre, I. Vlassiouk, Z.S. Siwy. Control of ionic transport through gated single conical nanopores. Analytical and bioanalytical chemistry, 2009, 394, 413.

[78] A. Asatekin, K.K. Gleason. Polymeric nanopore membranes for hydrophobicity-based separations by conformal initiated chemical vapor deposition. Nano Lett., 2011, 11, 677.

[79] C. Wolden, S. Kelkar. Evaluation of Vapor Deposition Techniques for Membrane Pore Size Modification. Journal of Membrane Science and Research, 2017, 3, 64.

[80] A. Asatekin, M.C. Barr, S.H. Baxamusa, K.K.S. Lau, W. Tenhaeff, J. Xu, K.K. Gleason. Designing polymer surfaces via vapor deposition. Materials Today, 2010, 13, 26.

[81] M. Weber, A. Julbe, A. Ayral, P. Miele, M. Bechelany. Atomic Layer Deposition for Membranes: Basics, Challenges, and Opportunities. Chem. Mater., 2018, 30, 7368.

[82] O. Graniel, M. Weber, S. Balme, P. Miele, M. Bechelany. Atomic layer deposition for biosensing applications. Biosensors \& bioelectronics, 2018, 122, 147.

[83] M. Weber, B. Koonkaew, S. Balme, I. Utke, F. Picaud, I. Iatsunskyi, E. Coy, P. Miele, M. Bechelany. Boron Nitride Nanoporous Membranes with High Surface Charge by Atomic Layer Deposition. ACS applied materials \& interfaces, 2017, 9, 16669.

[84] S. Cabello-Aguilar, S. Balme, A.A. Chaaya, M. Bechelany, E. Balanzat, J.-M. Janot, C. Pochat-Bohatier, P. Miele, P. Dejardin. Slow translocation of polynucleotides and their discrimination by $\alpha$-hemolysin inside a single track-etched nanopore designed by atomic layer deposition. Nanoscale, 2013, 5, 9582.

[85] M. Lepoitevin, M. Bechelany, E. Balanzat, J.-M. Janot, S. Balme. Non-Fluorescence label protein sensing with track-etched nanopore decorated by avidin/biotin system.

Electrochimica Acta, 2016, 211, 611.

[86] H. Li, D. Kong, Z. Wang, L. Yang, J. Xue, Q. Chen. Selective-area ALD for positively and negatively charged layers into the ion-beam track-etched conical pores in polyethylene terephthalate. AIP Advances, 2019, 9, 75114.

[87] N. Sobel, C. Hess, M. Lukas, A. Spende, B. Stühn, M.E. Toimil-Molares, C. Trautmann. Conformal SiO2 coating of sub-100 nm diameter channels of polycarbonate etched ion-track channels by atomic layer deposition. Beilstein journal of nanotechnology, 2015, 6, 472.

[88] A. Spende, N. Sobel, M. Lukas, R. Zierold, J.C. Riedl, L. Gura, I. Schubert, J.M.M. Moreno, K. Nielsch, B. Stühn, C. Hess, C. Trautmann, M.E. Toimil-Molares. TiO2, SiO2, and $\mathrm{A} 12 \mathrm{O} 3$ coated nanopores and nanotubes produced by ALD in etched ion-track membranes for transport measurements. Nanotechnology, 2015, 26, 335301.

[89] M. Leo, F.C. Pereira, L.M. Moretto, P. Scopece, S. Polizzi, P. Ugo. Towards a Better Understanding of Gold Electroless Deposition in Track-Etched Templates. Chem. Mater., 2007, 19, 5955.

[90] Y. Choi, L.A. Baker, H. Hillebrenner, C.R. Martin. Biosensing with conically shaped nanopores and nanotubes. Physical chemistry chemical physics : PCCP, 2006, 8, 4976. 


\section{WILEY-VCH}

[91] C.R. Martin, M. Nishizawa, K. Jirage, M. Kang. Investigations of the Transport Properties of Gold Nanotubule Membranes. J. Phys. Chem. B, 2001, 105, 1925.

[92] a) L.T. Sexton, L.P. Horne, C.R. Martin. Developing synthetic conical nanopores for biosensing applications. Molecular bioSystems, 2007, 3, 667; b) S.B. Lee, C.R. Martin. pHswitchable, ion-permselective gold nanotubule membrane based on chemisorbed cysteine. Analytical chemistry, 2001, 73, 768;

[93] K.-Y. Chun, S. Mafé, P. Ramírez, P. Stroeve. Protein transport through gold-coated, charged nanopores: Effects of applied voltage. Chemical Physics Letters, 2006, 418, 561.

[94] A. Asandei, F. Pintilie, T. Luchian. Romanian J. Biophys., 2006, 16, 273.

[95] P. Scopece, L.A. Baker, P. Ugo, C.R. Martin. Conical nanopore membranes: Solvent shaping of nanopores. Nanotechnology, 2006, 17, 3951.

[96] D. Ding, P. Gao, Q. Ma, D. Wang, F. Xia. Biomolecule-Functionalized Solid-State Ion Nanochannels/Nanopores: Features and Techniques. Small (Weinheim an der Bergstrasse, Germany), 2019, 15, e1804878.

[97] Z. Zhang, L. Wen, L. Jiang. Bioinspired smart asymmetric nanochannel membranes. Chemical Society reviews, 2018, 47, 322.

[98] G. Pérez-Mitta, A.G. Albesa, W. Knoll, C. Trautmann, M.E. Toimil-Molares, O. Azzaroni. Host-guest supramolecular chemistry in solid-state nanopores: Potassium-driven modulation of ionic transport in nanofluidic diodes. Nanoscale, 2015, 7, 15594.

[99] K. Wu, K. Xiao, L. Chen, R. Zhou, B. Niu, Y. Zhang, L. Wen. Biomimetic VoltageGated Ultrasensitive Potassium-Activated Nanofluidic Based on a Solid-State Nanochannel. Langmuir, 2017, 33, 8463.

[100] T. Ma, M. Walko, M. Lepoitevin, J.-M. Janot, E. Balanzat, A. Kocer, S. Balme. Combining Light-Gated and pH-Responsive Nanopore Based on PEG-Spiropyran Functionalization. Adv. Mater. Interfaces, 2018, 5, 1701051.

[101] Y. Gao, I. Kyratzis. Covalent immobilization of proteins on carbon nanotubes using the cross-linker 1-ethyl-3-(3-dimethylaminopropyl)carbodiimide--a critical assessment. Bioconjugate chemistry, 2008, 19, 1945.

[102] G.W. Anderson, J.E. Zimmerman, F.M. Callahan. The Use of Esters of NHydroxysuccinimide in Peptide Synthesis. J. Am. Chem. Soc., 1964, 86, 1839.

[103] M.C. Chang, W.H. Douglas. Cross-linkage of hydroxyapatite/gelatin nanocomposite using imide-based zero-length cross-linker. Journal of materials science. Materials in medicine, 2007, 18, 2045.

[104] S.R. Rasmussen, M.R. Larsen, S.E. Rasmussen. Covalent immobilization of DNA onto polystyrene microwells: The molecules are only bound at the $5^{\prime}$ end. Analytical Biochemistry, 1991, 198, 138.

[105] W.S. Choi, J.-H. Park, H.Y. Koo, J.-Y. Kim, B.K. Cho, D.-Y. Kim. "Grafting-from" polymerization inside a polyelectrolyte hollow-capsule microreactor. Angewandte Chemie (International ed. in English), 2005, 44, 1096.

[106] B. Zdyrko, I. Luzinov. Polymer brushes by the "grafting to" method. Macromol. Rapid Commun., 2011, 32, 859.

[107] a) K. Matyjaszewski. Atom Transfer Radical Polymerization (ATRP): Current Status and Future Perspectives. Macromolecules, 2012, 45, 4015; b) H. Gao, K. Matyjaszewski. Synthesis of Star Polymers by a Combination of ATRP and the "Click" Coupling Method. Macromolecules, 2006, 39, 4960; c) K.L. Robinson, M.A. Khan, M.V. Paz Báñez, X.S. Wang, S.P. Armes. Controlled Polymerization of 2-Hydroxyethyl Methacrylate by ATRP at Ambient Temperature. Macromolecules, 2001, 34, 3155;

[108] a) J. Chiefari, Y.K. Chong, F. Ercole, J. Krstina, J. Jeffery, T.P.T. Le, R.T.A. Mayadunne, G.F. Meijs, C.L. Moad, G. Moad, E. Rizzardo, S.H. Thang. Living Free-Radical Polymerization by Reversible Addition-Fragmentation Chain Transfer: The RAFT Process. Macromolecules, 1998, 31, 5559; b) G. Moad, Y.K. Chong, A. Postma, E. Rizzardo, S.H. 


\section{WILEY-VCH}

Thang. Advances in RAFT polymerization: The synthesis of polymers with defined endgroups. Polymer, 2005, 46, 8458; c) R.T.A. Mayadunne, E. Rizzardo, J. Chiefari, Y.K. Chong, G. Moad, S.H. Thang. Living Radical Polymerization with Reversible

Addition-Fragmentation Chain Transfer (RAFT Polymerization) Using Dithiocarbamates as Chain Transfer Agents. Macromolecules, 1999, 32, 6977;

[109] a) S. Edmondson, V.L. Osborne, W.T.S. Huck. Polymer brushes via surface-initiated polymerizations. Chemical Society reviews, 2004, 33, 14; b) S.H. Lee, D.R. Dreyer, J. An, A. Velamakanni, R.D. Piner, S. Park, Y. Zhu, S.O. Kim, C.W. Bielawski, R.S. Ruoff. Polymer Brushes via Controlled, Surface-Initiated Atom Transfer Radical Polymerization (ATRP) from Graphene Oxide. Macromol. Rapid Commun., 2010, 31, 281; c) C. Perruchot, M.A. Khan, A. Kamitsi, S.P. Armes, T. Werne, T.E. Patten. Synthesis of Well-Defined, PolymerGrafted Silica Particles by Aqueous ATRP. Langmuir, 2001, 17, 4479; d) M.H. Stenzel, L. Zhang, W.T.S. Huck. Temperature-Responsive Glycopolymer Brushes Synthesized via RAFT Polymerization Using the Z-group Approach. Macromol. Rapid Commun., 2006, 27, 1121; e) R. Ranjan, W.J. Brittain. Synthesis of High Density Polymer Brushes on Nanoparticles by Combined RAFT Polymerization and Click Chemistry. Macromol. Rapid Commun., 2008, 29, 1104;

[110] a) X. Fan, L. Lin, J.L. Dalsin, P.B. Messersmith. Biomimetic anchor for surfaceinitiated polymerization from metal substrates. J. Am. Chem. Soc., 2005, 127, 15843; b) R. Barbey, L. Lavanant, D. Paripovic, N. Schüwer, C. Sugnaux, S. Tugulu, H.-A. Klok. Polymer brushes via surface-initiated controlled radical polymerization: Synthesis, characterization, properties, and applications. Chemical reviews, 2009, 109, 5437;

[111] V. Coessens, T. Pintauer, K. Matyjaszewski. Functional polymers by atom transfer radical polymerization. Progress in Polymer Science, 2001, 26, 337.

[112] N. Ayres. Atom Transfer Radical Polymerization: A Robust and Versatile Route for Polymer Synthesis. Polymer Reviews, 2011, 51, 138.

[113] M. Barsbay, O. Güven. Grafting in confined spaces: Functionalization of nanochannels of track-etched membranes. Radiation Physics and Chemistry, 2014, 105, 26. [114] H. Alem, A.-S. Duwez, P. Lussis, P. Lipnik, A.M. Jonas, S. Demoustier-Champagne. Microstructure and thermo-responsive behavior of poly(N-isopropylacrylamide) brushes grafted in nanopores of track-etched membranes. Journal of Membrane Science, 2008, 308, 75.

[115] H. Alem, A.M. Jonas, S. Demoustier-Champagne. Poly(N-isopropylacrylamide) grafted into nanopores: Thermo-responsive behaviour in the presence of different salts. Polymer Degradation and Stability, 2010, 95, 327.

[116] W. Guo, H. Xia, F. Xia, X. Hou, L. Cao, L. Wang, J. Xue, G. Zhang, Y. Song, D. Zhu, Y. Wang, L. Jiang. Current rectification in temperature-responsive single nanopores.

Chemphyschem : a European journal of chemical physics and physical chemistry, 2010, 11, 859.

[117] C. Barner-Kowollik, S. Perrier. The future of reversible addition fragmentation chain transfer polymerization. J. Polym. Sci. A Polym. Chem., 2008, 46, 5715.

[118] M. Barsbay, O. Güven, H. Bessbousse, T.L. Wade, F. Beuneu, M.-C. Clochard. Nanopore size tuning of polymeric membranes using the RAFT-mediated radical polymerization. Journal of Membrane Science, 2013, 445, 135.

[119] H. Bessbousse, N. Zran, J. Fauléau, B. Godin, V. Lemée, T. Wade, M.-C. Clochard. Poly(4-vinyl pyridine) radiografted PVDF track etched membranes as sensors for monitoring trace mercury in water. Radiation Physics and Chemistry, 2016, 118, 48.

[120] M.V. Zdorovets, I.V. Korolkov, A.B. Yeszhanov, Y.G. Gorin. Functionalization of PET Track-Etched Membranes by UV-Induced Graft (co)Polymerization for Detection of Heavy Metal Ions in Water. Polymers, 2019, 11. 


\section{WILEY-VCH}

[121] a) M. Husseman, E.E. Malmström, M. McNamara, M. Mate, D. Mecerreyes, D.G. Benoit, J.L. Hedrick, P. Mansky, E. Huang, T.P. Russell, C.J. Hawker. Controlled Synthesis of Polymer Brushes by "Living" Free Radical Polymerization Techniques. Macromolecules, 1999, 32, 1424; b) R. Matsuno, K. Yamamoto, H. Otsuka, A. Takahara. Polystyrene- and Poly(3-vinylpyridine)-Grafted Magnetite Nanoparticles Prepared through Surface-Initiated Nitroxide-Mediated Radical Polymerization. Macromolecules, 2004, 37, 2203; c) M. Kobayashi, R. Matsuno, H. Otsuka, A. Takahara. Precise surface structure control of inorganic solid and metal oxide nanoparticles through surface-initiated radical polymerization. Science and Technology of Advanced Materials, 2016, 7, 617; d) X. Zhao, W. Lin, N. Song, X. Chen, X. Fan, Q. Zhou. Water soluble multi-walled carbon nanotubes prepared via nitroxidemediated radical polymerization. J. Mater. Chem., 2006, 16, 4619;

[122] a) R. Jordan, A. Ulman, J.F. Kang, M.H. Rafailovich, J. Sokolov. Surface-Initiated Anionic Polymerization of Styrene by Means of Self-Assembled Monolayers. J. Am. Chem. Soc., 1999, 121, 1016; b) M.D.K. Ingall, C.H. Honeyman, J.V. Mercure, P.A. Bianconi, R.R. Kunz. Surface Functionalization and Imaging Using Monolayers and Surface-Grafted Polymer Layers. J. Am. Chem. Soc., 1999, 121, 3607; c) R. Advincula, Q. Zhou, M. Park, S. Wang, J. Mays, G. Sakellariou, S. Pispas, N. Hadjichristidis. Polymer Brushes by Living Anionic Surface Initiated Polymerization on Flat Silicon ( $\mathrm{SiO} x$ ) and Gold Surfaces: Homopolymers and Block Copolymers. Langmuir, 2002, 18, 8672;

[123] a) P. Lambrinos, M. Tardi, A. Polton, P. Sigwalt. The mechanism of the polymerization of n.butyl acrylate initiated with N,N-diethyl dithiocarbamate derivatives. European Polymer Journal, 1990, 26, 1125; b) T. Otsu, M. Yoshida. Polymer design by iniferter technique in radical polymerization: synthesis of ab and aba block copolymers containing random and alternating copolymer sequences. Makromol. Chem., Rapid Commun., 1982, 3, 127; c) Y. Nakayama, T. Matsuda. Surface Macromolecular Architectural Designs Using Photo-Graft Copolymerization Based on Photochemistry of Benzyl N , N Diethyldithiocarbamate. Macromolecules, 1996, 29, 8622; d) T. Matsuda, M. Kaneko, S. Ge. Quasi-living surface graft polymerization with phosphorylcholine group(s) at the terminal end. Biomaterials, 2003, 24, 4507;

[124] a) N.Y. Kim, N.L. Jeon, I.S. Choi, S. Takami, Y. Harada, K.R. Finnie, G.S. Girolami, R.G. Nuzzo, G.M. Whitesides, P.E. Laibinis. Surface-Initiated Ring-Opening Metathesis Polymerization on Si/SiO 2. Macromolecules, 2000, 33, 2793; b) J.H. Moon, T.M. Swager. Poly( p -phenylene ethynylene) Brushes. Macromolecules, 2002, 35, 6086;

[125] a) R. Jordan, A. Ulman. Surface Initiated Living Cationic Polymerization of 2Oxazolines. J. Am. Chem. Soc., 1998, 120, 243; b) M. Husemann, D. Mecerreyes, C.J. Hawker, J.L. Hedrick, R. Shah, N.L. Abbott. Surface-Initiated Polymerization for Amplification of Self-Assembled Monolayers Patterned by Microcontact Printing. Angewandte Chemie (International ed. in English), 1999, 38, 647;

[126] G. Nguyen, S. Howorka, Z.S. Siwy. DNA strands attached inside single conical nanopores: Ionic pore characteristics and insight into DNA biophysics. The Journal of membrane biology, 2011, 239, 105.

[127] S. Nasir, M. Ali, W. Ensinger. Thermally controlled permeation of ionic molecules through synthetic nanopores functionalized with amine-terminated polymer brushes.

Nanotechnology, 2012, 23, 225502.

[128] M. Lepoitevin, G. Nguyen, M. Bechelany, E. Balanzat, J.-M. Janot, S. Balme. Combining a sensor and a $\mathrm{pH}$-gated nanopore based on an avidin-biotin system. Chemical communications (Cambridge, England), 2015, 51, 5994.

[129] a) G. Decher, J.-D. Hong. Buildup of ultrathin multilayer films by a self-assembly process, 1 consecutive adsorption of anionic and cationic bipolar amphiphiles on charged surfaces. Makromolekulare Chemie. Macromolecular Symposia, 1991, 46, 321; b) N.I. Kovtyukhova, P.J. Ollivier, B.R. Martin, T.E. Mallouk, S.A. Chizhik, E.V. Buzaneva, A.D. 


\section{WILEY-VCH}

Gorchinskiy. Layer-by-Layer Assembly of Ultrathin Composite Films from Micron-Sized Graphite Oxide Sheets and Polycations. Chem. Mater., 1999, 11, 771; c) N.A. Kotov, I. Dekany, J.H. Fendler. Layer-by-Layer Self-Assembly of Polyelectrolyte-Semiconductor Nanoparticle Composite Films. J. Phys. Chem., 1995, 99, 13065; d) G.B. Sukhorukov, E. Donath, H. Lichtenfeld, E. Knippel, M. Knippel, A. Budde, H. Möhwald. Layer-by-layer self assembly of polyelectrolytes on colloidal particles. Colloids and Surfaces A: Physicochemical and Engineering Aspects, 1998, 137, 253; e) Z. Tang, Y. Wang, P. Podsiadlo, N.A. Kotov. Biomedical Applications of Layer-by-Layer Assembly: From Biomimetics to Tissue Engineering. Adv. Mater., 2006, 18, 3203;

[130] a) T.D. Lazzara, K.H.A. Lau, A.I. Abou-Kandil, A.-M. Caminade, J.-P. Majoral, W. Knoll. Polyelectrolyte layer-by-layer deposition in cylindrical nanopores. ACS nano, 2010, 4, 3909; b) O. Azzaroni, K.H.A. Lau. Layer-by-Layer Assemblies in Nanoporous Templates: Nano-Organized Design and Applications of Soft Nanotechnology. Soft matter, 2011, 7 , 8709 ; c) M. Raoufi, D. Tranchida, H. Schönherr. Pushing the size limits in the replication of nanopores in anodized aluminum oxide via the layer-by-layer deposition of polyelectrolytes. Langmuir, 2012, 28, 10091; d) J. Song, D. Jańczewski, Y. Guo, J. Xu, G.J. Vancso. Redox responsive nanotubes from organometallic polymers by template assisted layer by layer fabrication. Nanoscale, 2013, 5, 11692; e) P. Actis, B. Vilozny, R.A. Seger, X. Li, O. Jejelowo, M. Rinaudo, N. Pourmand. Voltage-controlled metal binding on polyelectrolytefunctionalized nanopores. Langmuir, 2011, 27, 6528;

[131] M. Lepoitevin, B. Jamilloux, M. Bechelany, E. Balanzat, J.-M. Janot, S. Balme. Fast and reversible functionalization of a single nanopore based on layer-by-layer polyelectrolyte self-assembly for tuning current rectification and designing sensors. RSC Adv, 2016, 6, 32228. [132] H. Alem, F. Blondeau, K. Glinel, S. Demoustier-Champagne, A.M. Jonas. Layer-byLayer Assembly of Polyelectrolytes in Nanopores. Macromolecules, 2007, 40, 3366. [133] M. Ali, B. Yameen, J. Cervera, P. Ramírez, R. Neumann, W. Ensinger, W. Knoll, O. Azzaroni. Layer-by-Layer Assembly of Polyelectrolytes into Ionic Current Rectifying SolidState Nanopores: Insights from Theory and Experiment. J. Am. Chem. Soc., 2010, 132, 8338. [134] S. Balme, T. Ma, E. Balanzat, J.-M. Janot. Large osmotic energy harvesting from functionalized conical nanopore suitable for membrane applications. Journal of Membrane Science, 2017, 544, 18.

[135] a) K. Tamada, J. Nagasawa, F. Nakanishi, K. Abe, M. Hara, W. Knoll, T. Ishida, H. Fukushima, S. Miyashita, T. Usui, T. Koini, T. R. Lee. Structure of SAMs generated from functionalized thiols on gold. Thin Solid Films, 1998, 327-329, 150; b) V. Ganesh, S.K. Pal, S. Kumar, V. Lakshminarayanan. Self-assembled monolayers (SAMs) of alkoxycyanobiphenyl thiols on gold--a study of electron transfer reaction using cyclic voltammetry and electrochemical impedance spectroscopy. Journal of colloid and interface science, 2006, 296, 195 ;

[136] M. Pevarnik, K. Healy, M. Davenport, J. Yen, Z.S. Siwy. A hydrophobic entrance enhances ion current rectification and induces dewetting in asymmetric nanopores. The Analyst, 2012, 137, 2944.

[137] R. Wei, V. Gatterdam, R. Wieneke, R. Tampé, U. Rant. Stochastic sensing of proteins with receptor-modified solid-state nanopores. Nature nanotechnology, 2012, 7, 257.

[138] D. Coglitore, J.-M. Janot, S. Balme. Protein at liquid solid interfaces: Toward a new paradigm to change the approach to design hybrid protein/solid-state materials. Advances in colloid and interface science, 2019, 270, 278.

[139] T. Ma, J.-M. Janot, S. Balme. Dynamics of long hyaluronic acid chains through conical nanochannels for characterizing enzyme reactions in confined spaces. Nanoscale, 2020, 12, 7231. 


\section{WILEY-VCH}

[140] E.N. Vasina, P. Déjardin. Kinetics of adsorption, desorption, and exchange of alphachymotrypsin and lysozyme on poly(ethyleneterephthalate) tracked film and track-etched membrane. Biomacromolecules, 2003, 4, 304.

[141] M. Ali, V. Bayer, B. Schiedt, R. Neumann, W. Ensinger. Fabrication and functionalization of single asymmetric nanochannels for electrostatic/hydrophobic association of protein molecules. Nanotechnology, 2008, 19, 485711.

[142] M. Ali, S. Nasir, P. Ramirez, J. Cervera, S. Mafe, W. Ensinger. CarbohydrateMediated Biomolecular Recognition and Gating of Synthetic Ion Channels. J. Phys. Chem. C, 2013, 117, 18234.

[143] Q.H. Nguyen, M. Ali, R. Neumann, W. Ensinger. Saccharide/glycoprotein recognition inside synthetic ion channels modified with boronic acid. Sensors and Actuators B: Chemical, 2012, 162, 216.

[144] R.B. Schoch, J. Han, P. Renaud. Transport phenomena in nanofluidics. Rev. Mod. Phys., 2008, 80, 839.

[145] a) J.-L. Barrat, L. Bocquet. Large Slip Effect at a Nonwetting Fluid-Solid Interface. Phys. Rev. Lett., 1999, 82, 4671; b) L. Joly, C. Ybert, E. Trizac, L. Bocquet. Liquid friction on charged surfaces: From hydrodynamic slippage to electrokinetics. The Journal of chemical physics, 2006, 125, 204716;

[146] L. Bocquet, E. Charlaix. Nanofluidics, from bulk to interfaces. Chemical Society reviews, 2010, 39, 1073.

[147] E. Secchi, S. Marbach, A. Niguès, D. Stein, A. Siria, L. Bocquet. Massive radiusdependent flow slippage in carbon nanotubes. Nature, 2016, 537, 210.

[148] S. Balme, F. Picaud, M. Lepoitevin, M. Bechelany, E. Balanzat, J.-M. Janot. Unexpected ionic transport behavior in hydrophobic and uncharged conical nanopores.

Faraday discussions, 2018, 210, 69.

[149] Y. Tanaka. Ion Exchange Membranes Fundamentals and applications; Elsevier, 2007.

[150] E.C. Yusko, R. An, M. Mayer. Electroosmotic flow can generate ion current rectification in nano- and micropores. ACS nano, 2010, 4, 477.

[151] J.W. Jorgenson, K.D. Lukacs. High-resolution separations based on electrophoresis and electroosmosis. Journal of Chromatography A, 1981, 218, 209.

[152] K.M. Hong, J. Noolandi. Solution of the Smoluchowski equation with a Coulomb potential. I. General results. The Journal of chemical physics, 1978, 68, 5163.

[153] A. Siria, M.-L. Bocquet, L. Bocquet. New avenues for the large-scale harvesting of blue energy. Nat Rev Chem, 2017, 1, 294.

[154] S. Ghosal. Effect of salt concentration on the electrophoretic speed of a polyelectrolyte through a nanopore. Phys. Rev. Lett., 2007, 98, 238104.

[155] a) I. Huisman. Electroviscous effects, streaming potential, and zeta potential in polycarbonate track-etched membranes. Journal of Membrane Science, 2000, 178, 79; b) C. Lettmann, D. Möckel, E. Staude. Permeation and tangential flow streaming potential measurements for electrokinetic characterization of track-etched microfiltration membranes. Journal of Membrane Science, 1999, 159, 243;

[156] K. Kim. Chemical and electrical characterization of virgin and protein-fouled polycarbonate track-etched membranes by FTIR and streaming-potential measurements. Journal of Membrane Science, 1997, 134, 199.

[157] a) W.H. Keesom, R.L. Zelenka, C.J. Radke. A zeta-potential model for ionic surfactant adsorption on an ionogenic hydrophobic surface. Journal of colloid and interface science, 1988, 125, 575; b) J.I. Calvo, A. Hernández, P. Prádanos, F. Tejerina. Charge Adsorption and Zeta Potential in Cyclopore Membranes. Journal of colloid and interface science, 1996, 181, 399 ;

[158] H. Strathmann. Ion-Exchange Membrane Separation Processes; Elsevier: 2004. 


\section{WILEY-VCH}

[159] K. Kontturi, L. Murtomäki, J.A. Manzanares. Ionic Transport Processes: in Electrochemistry and Membrane Science; OUP Oxford, 2008.

[160] Q. Liu, Y. Wang, W. Guo, H. Ji, J. Xue, Q. Ouyang. Asymmetric properties of ion transport in a charged conical nanopore. Physical review. E, Statistical, nonlinear, and soft matter physics, 2007, 75, 51201.

[161] a) G. Mitscha-Baude, A. Buttinger-Kreuzhuber, G. Tulzer, C. Heitzinger. Adaptive and iterative methods for simulations of nanopores with the PNP-Stokes equations. Journal of Computational Physics, 2017, 338, 452; b) H.-C. Yeh, C.-C. Chang, R.-J. Yang. Reverse electrodialysis in conical-shaped nanopores: Salinity gradient-driven power generation. $R S C$ $A d v, \mathbf{2 0 1 4}, 4,2705$; c) D.-H. Lin, C.-Y. Lin, S. Tseng, J.-P. Hsu. Influence of electroosmotic flow on the ionic current rectification in a pH-regulated, conical nanopore. Nanoscale, 2015, 7, 14023;

[162] J.-P. Hsu, S.-T. Yang, C.-Y. Lin, S. Tseng. Ionic Current Rectification in a Conical Nanopore: Influences of Electroosmotic Flow and Type of Salt. J. Phys. Chem. C, 2017, 121, 4576.

[163] a) Y. Ai, M. Zhang, S.W. Joo, M.A. Cheney, S. Qian. Effects of Electroosmotic Flow on Ionic Current Rectification in Conical Nanopores. J. Phys. Chem. C, 2010, 114, 3883; b) A. Szymczyk, H. Zhu, B. Balannec. Ion rejection properties of nanopores with bipolar fixed charge distributions. The journal of physical chemistry. B, 2010, 114, 10143;

[164] D. Stein, M. Kruithof, C. Dekker. Surface-charge-governed ion transport in nanofluidic channels. Phys. Rev. Lett., 2004, 93, 35901.

[165] S. Balme, F. Picaud, M. Manghi, J. Palmeri, M. Bechelany, S. Cabello-Aguilar, A. Abou-Chaaya, P. Miele, E. Balanzat, J.M. Janot. Ionic transport through sub-10 nm diameter hydrophobic high-aspect ratio nanopores: Experiment, theory and simulation. Scientific reports, 2015, 5, 10135.

[166] M. Tagliazucchi, I. Szleifer. Theoretical Basis for Structure and Transport in Nanopores and Nanochannels. In: Chemically Modified Nanopores and Nanochannels. Tagliazucchi, M., Szleifer, I., Eds.; Elsevier, 2017; pp. 27-60.

[167] Y. Yan, L. Wang, J. Xue, H.-C. Chang. Ion current rectification inversion in conic nanopores: Nonequilibrium ion transport biased by ion selectivity and spatial asymmetry. The Journal of chemical physics, 2013, 138, 44706.

[168] I. Vlassiouk, Z.S. Siwy. Nanofluidic diode. Nano Lett., 2007, 7, 552.

[169] P. Ramírez, V. Gómez, J. Cervera, B. Schiedt, S. Mafé. Ion transport and selectivity in nanopores with spatially inhomogeneous fixed charge distributions. The Journal of chemical physics, 2007, 126, 194703.

[170] Z. Siwy, I.D. Kosińska, A. Fuliński, C.R. Martin. Asymmetric diffusion through synthetic nanopores. Phys. Rev. Lett., 2005, 94, 48102.

[171] P. Ramírez, P.Y. Apel, J. Cervera, S. Mafé. Pore structure and function of synthetic nanopores with fixed charges: Tip shape and rectification properties. Nanotechnology, 2008, $19,315707$.

[172] I. Vlassiouk, S. Smirnov, Z. Siwy. Ionic selectivity of single nanochannels. Nano Lett., 2008, 8, 1978.

[173] J.-F. Pietschmann, M.-T. Wolfram, M. Burger, C. Trautmann, G. Nguyen, M.

Pevarnik, V. Bayer, Z. Siwy. Rectification properties of conically shaped nanopores:

Consequences of miniaturization. Physical chemistry chemical physics : $P C C P, \mathbf{2 0 1 3}, 15$,

16917.

[174] S. Tseng, S.-C. Lin, C.-Y. Lin, J.-P. Hsu. Influences of Cone Angle and Surface

Charge Density on the Ion Current Rectification Behavior of a Conical Nanopore. J. Phys.

Chem. C, 2016, 120, 25620.

[175] Tagliazucchi, M.; Szleifer, I., Eds. Chemically Modified Nanopores and

Nanochannels; Elsevier, 2017. 


\section{WILEY-VCH}

[176] Y. He, D. Gillespie, D. Boda, I. Vlassiouk, R.S. Eisenberg, Z.S. Siwy. Tuning transport properties of nanofluidic devices with local charge inversion. J. Am. Chem. Soc., 2009, 131, 5194.

[177] H. Daiguji, Y. Oka, K. Shirono. Nanofluidic diode and bipolar transistor. Nano Lett., 2005, 5, 2274.

[178] K. Pal Singh, K. Kumari, M. Kumar. Ion current rectification in a fluidic bipolar nanochannel with smooth junction. Appl. Phys. Lett., 2011, 99, 113103.

[179] K. Pal Singh, M. Kumar. Effect of surface charge density and electro-osmotic flow on ionic current in a bipolar nanopore fluidic diode. Journal of Applied Physics, 2011, 110, 84322.

[180] C.-Y. Lin, T. Ma, Z.S. Siwy, S. Balme, J.-P. Hsu. Tunable Current Rectification and Selectivity Demonstrated in Nanofluidic Diodes through Kinetic Functionalization. The journal of physical chemistry letters, 2020, 11, 60.

[181] L. Cao, W. Guo, Y. Wang, L. Jiang. Concentration-gradient-dependent ion current rectification in charged conical nanopores. Langmuir, 2012, 28, 2194.

[182] J.-P. Hsu, Y.-M. Chen, S.-T. Yang, C.-Y. Lin, S. Tseng. Influence of salt valence on the rectification behavior of nanochannels. Journal of colloid and interface science, 2018, 531, 483.

[183] X. He, K. Zhang, Y. Liu, F. Wu, P. Yu, L. Mao. Chaotropic Monovalent AnionInduced Rectification Inversion at Nanopipettes Modified by Polyimidazolium Brushes. Angewandte Chemie (International ed. in English), 2018, 57, 4590.

[184] W.-J. Lan, D.A. Holden, H.S. White. Pressure-dependent ion current rectification in conical-shaped glass nanopores. J. Am. Chem. Soc., 2011, 133, 13300.

[185] D. Momotenko, H.H. Girault. Scan-rate-dependent ion current rectification and rectification inversion in charged conical nanopores. J. Am. Chem. Soc., 2011, 133, 14496. [186] a) M. Davenport, A. Rodriguez, K.J. Shea, Z.S. Siwy. Squeezing ionic liquids through nanopores. Nano Lett., 2009, 9, 2125; b) X. Jiang, Y. Liu, R. Qiao. Current Rectification for Transport of Room-Temperature Ionic Liquids through Conical Nanopores. J. Phys. Chem. C, 2016, 120, 4629;

[187] a) X. Yin, S. Zhang, Y. Dong, S. Liu, J. Gu, Y. Chen, X. Zhang, X. Zhang, Y. Shao. Ionic current rectification in organic solutions with quartz nanopipettes. Analytical chemistry, 2015, 87, 9070; b) T. Plett, W. Shi, Y. Zeng, W. Mann, I. Vlassiouk, L.A. Baker, Z.S. Siwy. Rectification of nanopores in aprotic solvents--transport properties of nanopores with surface dipoles. Nanoscale, 2015, 7, 19080; c) R.A. Lucas, C.-Y. Lin, Z.S. Siwy. Electrokinetic Phenomena in Organic Solvents. The journal of physical chemistry. B, 2019, 123, 6123;

[188] K. Han, L. Heng, L. Wen, L. Jiang. Biomimetic heterogeneous multiple ion channels: A honeycomb structure composite film generated by breath figures. Nanoscale, 2016, 8 , 12318.

[189] J.-P. Hsu, Y.-C. Chen, Y.-M. Chen, S. Tseng. Influence of temperature and electroosmotic flow on the rectification behavior of conical nanochannels. Journal of the Taiwan Institute of Chemical Engineers, 2018, 93, 142.

[190] a) Z. Siwy, P. Apel, D. Dobrev, R. Neumann, R. Spohr, C. Trautmann, K. Voss. Ion transport through asymmetric nanopores prepared by ion track etching. Nuclear Instruments and Methods in Physics Research Section B: Beam Interactions with Materials and Atoms, 2003, 208, 143; b) L. Wang, W. Guo, Y.B. Xie, X.W. Wang, J.M. Xue, Y.G. Wang. Nanofluidic diode generated by $\mathrm{pH}$ gradient inside track-etched conical nanopore. Radiation Measurements, 2009, 44, 1119;

[191] J.-P. Hsu, H.-H. Wu, C.-Y. Lin, S. Tseng. Ion Current Rectification Behavior of Bioinspired Nanopores Having a pH-Tunable Zwitterionic Surface. Analytical chemistry, 2017, 89, 3952. 


\section{WILEY-VCH}

[192] G. Pérez-Mitta, J.S. Tuninetti, W. Knoll, C. Trautmann, M.E. Toimil-Molares, O. Azzaroni. Polydopamine meets solid-state nanopores: A bioinspired integrative surface chemistry approach to tailor the functional properties of nanofluidic diodes. J. Am. Chem. Soc., 2015, 137, 6011.

[193] F. Xia, W. Guo, Y. Mao, X. Hou, J. Xue, H. Xia, L. Wang, Y. Song, H. Ji, Q. Ouyang, Y. Wang, L. Jiang. Gating of single synthetic nanopores by proton-driven DNA molecular motors. J. Am. Chem. Soc., 2008, 130, 8345.

[194] B. Yameen, M. Ali, R. Neumann, W. Ensinger, W. Knoll, O. Azzaroni. Synthetic proton-gated ion channels via single solid-state nanochannels modified with responsive polymer brushes. Nano Lett., 2009, 9, 2788.

[195] B. Yameen, M. Ali, R. Neumann, W. Ensinger, W. Knoll, O. Azzaroni. Single conical nanopores displaying $\mathrm{pH}$-tunable rectifying characteristics. manipulating ionic transport with zwitterionic polymer brushes. J. Am. Chem. Soc., 2009, 131, 2070.

[196] G. Pérez-Mitta, L. Burr, J.S. Tuninetti, C. Trautmann, M.E. Toimil-Molares, O. Azzaroni. Noncovalent functionalization of solid-state nanopores via self-assembly of amphipols. Nanoscale, 2016, 8, 1470.

[197] G. Pérez-Mitta, W.A. Marmisolle, L. Burr, M.E. Toimil-Molares, C. Trautmann, O. Azzaroni. Proton-Gated Rectification Regimes in Nanofluidic Diodes Switched by Chemical Effectors. Small (Weinheim an der Bergstrasse, Germany), 2018, 14, e1703144.

[198] X. Hou, Y. Liu, H. Dong, F. Yang, L. Li, L. Jiang. A pH-gating ionic transport nanodevice: Asymmetric chemical modification of single nanochannels. Advanced materials (Deerfield Beach, Fla.), 2010, 22, 2440.

[199] S.F. Buchsbaum, G. Nguyen, S. Howorka, Z.S. Siwy. DNA-modified polymer pores allow pH- and voltage-gated control of channel flux. J. Am. Chem. Soc., 2014, 136, 9902. [200] G. Pérez-Mitta, W.A. Marmisollé, C. Trautmann, M.E. Toimil-Molares, O. Azzaroni. Nanofluidic Diodes with Dynamic Rectification Properties Stemming from Reversible Electrochemical Conversions in Conducting Polymers. J. Am. Chem. Soc., 2015, 137, 15382. [201] G. Pérez-Mitta, W.A. Marmisollé, C. Trautmann, M.E. Toimil-Molares, O. Azzaroni. An All-Plastic Field-Effect Nanofluidic Diode Gated by a Conducting Polymer Layer. Advanced materials (Deerfield Beach, Fla.), 2017, 29.

[202] N.K. Mal, M. Fujiwara, Y. Tanaka. Photocontrolled reversible release of guest molecules from coumarin-modified mesoporous silica. Nature, 2003, 421, 350.

[203] Liu, D.R. Dunphy, P. Atanassov, S.D. Bunge, Z. Chen, G.P. López, T.J. Boyle, C.J. Brinker. Photoregulation of Mass Transport through a Photoresponsive Azobenzene-Modified Nanoporous Membrane. Nano Lett., 2004, 4, 551.

[204] A. Koçer, M. Walko, W. Meijberg, B.L. Feringa. A light-actuated nanovalve derived from a channel protein. Science (New York, N.Y.), 2005, 309, 755.

[205] I. Vlassiouk, C.-D. Park, S.A. Vail, D. Gust, S. Smirnov. Control of nanopore wetting by a photochromic spiropyran: A light-controlled valve and electrical switch. Nano Lett.,

2006, 6, 1013.

[206] K. Xiao, X.-Y. Kong, Z. Zhang, G. Xie, L. Wen, L. Jiang. Construction and application of photoresponsive smart nanochannels. Journal of Photochemistry and Photobiology C: Photochemistry Reviews, 2016, 26, 31.

[207] P. Li, G. Xie, X.-Y. Kong, Z. Zhang, K. Xiao, L. Wen, L. Jiang. Light-Controlled Ion Transport through Biomimetic DNA-Based Channels. Angewandte Chemie (International ed. in English), 2016, 55, 15637.

[208] M. Zhang, X. Hou, J. Wang, Y. Tian, X. Fan, J. Zhai, L. Jiang. Light and pH cooperative nanofluidic diode using a spiropyran-functionalized single nanochannel. Advanced materials (Deerfield Beach, Fla.), 2012, 24, 2424. 


\section{WILEY-VCH}

[209] T. Qian, H. Zhang, X. Li, J. Hou, C. Zhao, Q. Gu, H. Wang. Efficient Gating of Ion Transport in Three - Dimensional Metal - Organic Framework Sub - Nanochannels with Confined Light - Responsive Azobenzene Molecules. Angew. Chem., 2020.

[210] L. Wen, Q. Liu, J. Ma, Y. Tian, C. Li, Z. Bo, L. Jiang. Malachite green derivativefunctionalized single nanochannel: Light-and-pH dual-driven ionic gating. Advanced materials (Deerfield Beach, Fla.), 2012, 24, 6193.

[211] a) X.-Z. Zhang, D.-Q. Wu, C.-C. Chu. Synthesis, characterization and controlled drug release of thermosensitive IPN-PNIPAAm hydrogels. Biomaterials, 2004, 25, 3793; b) T.

Peng, Y.-L. Cheng. Temperature-responsive permeability of porous PNIPAAm-g-PE membranes. J. Appl. Polym. Sci., 1998, 70, 2133;

[212] N. Liu, C. Li, T. Zhang, R. Hou, Z. Xiong, Z. Li, B. Wei, Z. Yang, P. Gao, X. Lou, X. Zhang, W. Guo, F. Xia. Fabrication of "Plug and Play" Channels with Dual Responses by Host-Guest Interactions. Small (Weinheim an der Bergstrasse, Germany), 2017, 13.

[213] Z. Zhang, G. Xie, K. Xiao, X.-Y. Kong, P. Li, Y. Tian, L. Wen, L. Jiang. Asymmetric Multifunctional Heterogeneous Membranes for $\mathrm{pH}$ - and Temperature-Cooperative Smart Ion Transport Modulation. Advanced materials (Deerfield Beach, Fla.), 2016, 28, 9613.

[214] R. Wang, Y. Sun, F. Zhang, M. Song, D. Tian, H. Li. Temperature-Sensitive Artificial Channels through Pillar5arene-based Host-Guest Interactions. Angewandte Chemie (International ed. in English), 2017, 56, 5294.

[215] Q. Liu, K. Xiao, L. Wen, H. Lu, Y. Liu, X.-Y. Kong, G. Xie, Z. Zhang, Z. Bo, L. Jiang. Engineered Ionic Gates for Ion Conduction Based on Sodium and Potassium Activated Nanochannels. J. Am. Chem. Soc., 2015, 137, 11976.

[216] M. Ali, I. Ahmed, P. Ramirez, S. Nasir, S. Mafe, C.M. Niemeyer, W. Ensinger. Lithium Ion Recognition with Nanofluidic Diodes through Host-Guest Complexation in Confined Geometries. Analytical chemistry, 2018, 90, 6820.

[217] M. Ali, I. Ahmed, S. Nasir, I. Duznovic, C.M. Niemeyer, W. Ensinger. Potassiuminduced ionic conduction through a single nanofluidic pore modified with acyclic polyether derivative. Analytica chimica acta, 2018, 1039, 132.

[218] M. Ali, I. Ahmed, P. Ramirez, S. Nasir, J. Cervera, S. Mafe, C.M. Niemeyer, W. Ensinger. Cesium-Induced Ionic Conduction through a Single Nanofluidic Pore Modified with Calixcrown Moieties. Langmuir, 2017, 33, 9170.

[219] X. Hou, W. Guo, F. Xia, F.-Q. Nie, H. Dong, Y. Tian, L. Wen, L. Wang, L. Cao, Y. Yang, J. Xue, Y. Song, Y. Wang, D. Liu, L. Jiang. A biomimetic potassium responsive nanochannel: G-quadruplex DNA conformational switching in a synthetic nanopore. J. Am. Chem. Soc., 2009, 131, 7800.

[220] Y. Tian, X. Hou, L. Wen, W. Guo, Y. Song, H. Sun, Y. Wang, L. Jiang, D. Zhu. A biomimetic zinc activated ion channel. Chemical communications (Cambridge, England), 2010, 46, 1682.

[221] Y. Tian, Z. Zhang, L. Wen, J. Ma, Y. Zhang, W. Liu, J. Zhai, L. Jiang. A biomimetic mercury(II)-gated single nanochannel. Chemical communications (Cambridge, England), 2013, 49, 10679.

[222] Y. Shang, Y. Zhang, P. Li, J. Lai, X.-Y. Kong, W. Liu, K. Xiao, G. Xie, Y. Tian, L. Wen, L. Jiang. DNAzyme tunable lead(II) gating based on ion-track etched conical nanochannels. Chemical communications (Cambridge, England), 2015, 51, 5979.

[223] X. Xu, R. Hou, P. Gao, M. Miao, X. Lou, B. Liu, F. Xia. Highly Robust NanoporeBased Dual-Signal-Output Ion Detection System for Achieving Three Successive Calibration Curves. Analytical chemistry, 2016, 88, 2386.

[224] F. Picaud, S. Kraszewski, C. Ramseyer, S. Balme, P. Déjardin, J.M. Janot, F. Henn. Enhanced potassium selectivity in a bioinspired solid nanopore. Physical chemistry chemical physics : PCCP, 2013, 15, 19601. 


\section{WILEY-VCH}

[225] F. Picaud, K. Boukari, S. Balme, J.M. Janot, F. Henn. Selectivity against proton in cation permeable hybrid solid state membrane. Computational Materials Science, 2016, 111, 380 .

[226] S. Balme, D. Thiele, S. Kraszewski, F. Picaud, J.-M. Janot, P. Déjardin. Ionic selectivity of nystatin A1 confined in nanoporous track-etched polymer membrane. IET nanobiotechnology, 2014, 8, 138.

[227] K. Boukari, G. Paris, T. Gharbi, S. Balme, J.-M. Janot, F. Picaud. Confined Nystatin

Polyenes in Nanopore Induce Biologic Ionic Selectivity. Journal of Nanomaterials, 2016, 2016, 1.

[228] J. Lu, H. Zhang, J. Hou, X. Li, X. Hu, Y. Hu, C.D. Easton, Q. Li, C. Sun, A.W. Thornton, M.R. Hill, X. Zhang, G. Jiang, J.Z. Liu, A.J. Hill, B.D. Freeman, L. Jiang, H. Wang. Efficient metal ion sieving in rectifying subnanochannels enabled by metal-organic frameworks. Nature materials, 2020.

[229] X. Li, H. Zhang, P. Wang, J. Hou, J. Lu, C.D. Easton, X. Zhang, M.R. Hill, A.W. Thornton, J.Z. Liu, B.D. Freeman, A.J. Hill, L. Jiang, H. Wang. Fast and selective fluoride ion conduction in sub-1-nanometer metal-organic framework channels. Nature communications, 2019, 10, 2490.

[230] H. Bayley, C.R. Martin. Resistive-Pulse Sensing-From Microbes to Molecules. Chemical reviews, 2000, 100, 2575.

[231] S. Garaj, W. Hubbard, A. Reina, J. Kong, D. Branton, J.A. Golovchenko. Graphene as a subnanometre trans-electrode membrane. Nature, 2010, 467, 190.

[232] J. Feng, K. Liu, R.D. Bulushev, S. Khlybov, D. Dumcenco, A. Kis, A. Radenovic. Identification of single nucleotides in MoS2 nanopores. Nature nanotechnology, 2015, 10, 1070 .

[233] A. Mara, Z. Siwy, C. Trautmann, J. Wan, F. Kamme. An Asymmetric Polymer Nanopore for Single Molecule Detection. Nano Lett., 2004, 4, 497.

[234] C.C. Harrell, Y. Choi, L.P. Horne, L.A. Baker, Z.S. Siwy, C.R. Martin. Resistivepulse DNA detection with a conical nanopore sensor. Langmuir: the ACS journal of surfaces and colloids, 2006, 22, 10837.

[235] S. Cabello-Aguilar, A.A. Chaaya, M. Bechelany, C. Pochat-Bohatier, E. Balanzat, J.M. Janot, P. Miele, S. Balme. Dynamics of polymer nanoparticles through a single artificial nanopore with a high-aspect-ratio. Soft matter, 2014, 10, 8413.

[236] M. Schiel, Z.S. Siwy. Diffusion and Trapping of Single Particles in Pores with Combined Pressure and Dynamic Voltage. J. Phys. Chem. C, 2014, 118, 19214.

[237] J. Menestrina, C. Yang, M. Schiel, I. Vlassiouk, Z.S. Siwy. Charged Particles Modulate Local Ionic Concentrations and Cause Formation of Positive Peaks in ResistivePulse-Based Detection. J. Phys. Chem. C, 2014, 118, 2391.

[238] R.M.M. Smeets, U.F. Keyser, D. Krapf, M.-Y. Wu, N.H. Dekker, C. Dekker. Salt dependence of ion transport and DNA translocation through solid-state nanopores. Nano Lett., 2006, 6, 89.

[239] Y. Qiu, C.-Y. Lin, P. Hinkle, T.S. Plett, C. Yang, J.V. Chacko, M.A. Digman, L.-H. Yeh, J.-P. Hsu, Z.S. Siwy. Highly Charged Particles Cause a Larger Current Blockage in Micropores Compared to Neutral Particles. ACS nano, 2016, 10, 8413.

[240] Y. Qiu, Z. Siwy. Probing charges on solid-liquid interfaces with the resistive-pulse technique. Nanoscale, 2017, 9, 13527.

[241] M. Pevarnik, M. Schiel, K. Yoshimatsu, I.V. Vlassiouk, J.S. Kwon, K.J. Shea, Z.S. Siwy. Particle deformation and concentration polarization in electroosmotic transport of hydrogels through pores. ACS nano, 2013, 7, 3720.

[242] Y. Qiu, P. Hinkle, C. Yang, H.E. Bakker, M. Schiel, H. Wang, D. Melnikov, M. Gracheva, M.E. Toimil-Molares, A. Imhof, Z.S. Siwy. Pores with longitudinal irregularities distinguish objects by shape. ACS nano, 2015, 9, 4390. 


\section{WILEY-VCH}

[243] M. Pevarnik, K. Healy, M.E. Toimil-Molares, A. Morrison, S.E. Létant, Z.S. Siwy. Polystyrene particles reveal pore substructure as they translocate. ACS nano, 2012, 6, 7295. [244] a) F. Picaud, G. Paris, T. Gharbi, M. Lepoitevin, P.-E. Coulon, M. Bechelany, J.M. Janot, S. Balme. Discrimination of Polynucleotide Transport through a Highly Hydrophobic Uncharged Nanopore. J. Phys. Chem. C, 2017, 121, 7525; b) M. Lepoitevin, P.E. Coulon, M. Bechelany, J. Cambedouzou, J.-M. Janot, S. Balme. Influence of nanopore surface charge and magnesium ion on polyadenosine translocation. Nanotechnology, 2015, 26, 144001;

[245] a) H. Lu, F. Giordano, Z. Ning. Oxford Nanopore MinION Sequencing and Genome Assembly. Genomics, proteomics \& bioinformatics, 2016, 14, 265; b) R.R. Wick, L.M. Judd, C.L. Gorrie, K.E. Holt. Completing bacterial genome assemblies with multiplex MinION sequencing. Microbial genomics, 2017, 3, e000132; c) A.D. Tyler, L. Mataseje, C.J. Urfano, L. Schmidt, K.S. Antonation, M.R. Mulvey, C.R. Corbett. Evaluation of Oxford Nanopore's MinION Sequencing Device for Microbial Whole Genome Sequencing Applications. Scientific reports, 2018, 8,10931 ;

[246] a) J. Li, D. Yu, Q. Zhao. Solid-state nanopore-based DNA single molecule detection and sequencing. Microchim Acta, 2016, 183, 941; b) P.M. Das. Recent Progress in Solid-State Nanopore DNA Sequencing. Biophysical Journal, 2020, 118, 158a;

[247] K. Kececi, N. San, D. Kaya. Nanopore detection of double stranded DNA using a track-etched polycarbonate membrane. Talanta, 2015, 144, 268.

[248] D. Kaya, A. Dinler, N. San, K. Kececi. Effect of Pore Geometry on Resistive-Pulse Sensing of DNA Using Track-Etched PET Nanopore Membrane. Electrochimica Acta, 2016, 202, 157.

[249] A. Dinler, K. Kececi, D. Kaya, D. Yilmaz. Investigating the Effect of Charged Components on Translocation of DNA Molecules in Track-Etched Nanopores. IEEE Sensors J., 2020, 20, 4041.

[250] J. Bentin, S. Balme, F. Picaud. Polynucleotide differentiation using hybrid solid-state nanopore functionalizing with $\alpha$-hemolysin. Soft matter, 2020, 16, 1002.

[251] a) E.C. Yusko, B.R. Bruhn, O.M. Eggenberger, J. Houghtaling, R.C. Rollings, N.C. Walsh, S. Nandivada, M. Pindrus, A.R. Hall, D. Sept, J. Li, D.S. Kalonia, M. Mayer. Realtime shape approximation and fingerprinting of single proteins using a nanopore. Nature nanotechnology, 2017, 12, 360; b) E.C. Yusko, J.M. Johnson, S. Majd, P. Prangkio, R.C. Rollings, J. Li, J. Yang, M. Mayer. Controlling protein translocation through nanopores with bio-inspired fluid walls. Nature nanotechnology, 2011, 6, 253; c) N. Varongchayakul, J. Song, A. Meller, M.W. Grinstaff. Single-molecule protein sensing in a nanopore: A tutorial. Chemical Society reviews, 2018, 47, 8512; [252] J.E. Wharton, P. Jin, L.T. Sexton, L.P. Horne, S.A. Sherrill, W.K. Mino, C.R. Martin. A method for reproducibly preparing synthetic nanopores for resistive-pulse biosensors. Small (Weinheim an der Bergstrasse, Germany), 2007, 3, 1424.

[253] L.T. Sexton, L.P. Horne, S.A. Sherrill, G.W. Bishop, L.A. Baker, C.R. Martin. Resistive-pulse studies of proteins and protein/antibody complexes using a conical nanotube sensor. J. Am. Chem. Soc., 2007, 129, 13144.

[254] L.T. Sexton, H. Mukaibo, P. Katira, H. Hess, S.A. Sherrill, L.P. Horne, C.R. Martin. An adsorption-based model for pulse duration in resistive-pulse protein sensing. J. Am. Chem. Soc., 2010, 132, 6755.

[255] J. Larkin, R.Y. Henley, M. Muthukumar, J.K. Rosenstein, M. Wanunu. Highbandwidth protein analysis using solid-state nanopores. Biophysical Journal, 2014, 106, 696. [256] Y. Zhang, X. Chen, C. Wang, G.M. Roozbahani, H.-C. Chang, X. Guan. Chemically functionalized conical PET nanopore for protein detection at the single-molecule level. Biosensors and Bioelectronics, 2020, 165, 112289.

[257] M.R. Powell, C. Martens, Z.S. Siwy. Asymmetric properties of ion current 1/f noise in conically shaped nanopores. Chemical Physics, 2010, 375, 529. 


\section{WILEY-VCH}

[258] N. Giamblanco, D. Coglitore, A. Gubbiotti, T. Ma, E. Balanzat, J.-M. Janot, M. Chinappi, S. Balme. Amyloid Growth, Inhibition, and Real-Time Enzymatic Degradation Revealed with Single Conical Nanopore. Analytical chemistry, 2018, 90, 12900.

[259] N. Giamblanco, Y. Fichou, J.-M. Janot, E. Balanzat, S. Han, S. Balme. Mechanisms of Heparin-Induced Tau Aggregation Revealed by a Single Nanopore. ACS sensors, 2020.

[260] N. Giamblanco, J. - M. Janot, A. Gubbiotti, M. Chinappi, S. Balme. Characterization of Food Amyloid Protein Digestion by Conical Nanopore. Small Methods, 2020, 9, 1900703.

[261] a) F. Chiti, C.M. Dobson. Protein Misfolding, Amyloid Formation, and Human Disease: A Summary of Progress Over the Last Decade. Annual review of biochemistry, 2017, 86, 27; b) T.P.J. Knowles, M. Vendruscolo, C.M. Dobson. The amyloid state and its association with protein misfolding diseases. Nature reviews. Molecular cell biology, 2014, 15,384

[262] S. Giorgetti, C. Greco, P. Tortora, F.A. Aprile. Targeting Amyloid Aggregation: An Overview of Strategies and Mechanisms. International journal of molecular sciences, 2018, 19.

[263] P. Li, X.-Y. Kong, G. Xie, K. Xiao, Z. Zhang, L. Wen, L. Jiang. Adenosine-Activated Nanochannels Inspired by G-Protein-Coupled Receptors. Small (Weinheim an der Bergstrasse, Germany), 2016, 12, 1854.

[264] J. Wang, J. Hou, H. Zhang, Y. Tian, L. Jiang. Single Nanochannel-Aptamer-Based Biosensor for Ultrasensitive and Selective Cocaine Detection. ACS applied materials \& interfaces, 2018, 10, 2033.

[265] M. Ali, S. Nasir, W. Ensinger. Stereoselective detection of amino acids with proteinmodified single asymmetric nanopores. Electrochimica Acta, 2016, 215, 231.

[266] M. Ali, I. Ahmed, S. Nasir, P. Ramirez, C.M. Niemeyer, S. Mafe, W. Ensinger. Ionic Transport through Chemically Functionalized Hydrogen Peroxide-Sensitive Asymmetric Nanopores. ACS applied materials \& interfaces, 2015, 7, 19541.

[267] K. Xiao, K. Wu, L. Chen, X.-Y. Kong, Y. Zhang, L. Wen, L. Jiang. Biomimetic Peptide-Gated Nanoporous Membrane for On-Demand Molecule Transport. Angew. Chem., 2018, 130, 157.

[268] M. Ali, R. Neumann, W. Ensinger. Sequence-specific recognition of DNA oligomer using peptide nucleic acid (PNA)-modified synthetic ion channels: PNA/DNA hybridization in nanoconfined environment. ACS nano, 2010, 4, 7267.

[269] Z. Sun, T. Liao, Y. Zhang, J. Shu, H. Zhang, G.-J. Zhang. Biomimetic nanochannels based biosensor for ultrasensitive and label-free detection of nucleic acids. Biosensors \& bioelectronics, 2016, 86, 194.

[270] T. Liao, X. Li, Q. Tong, K. Zou, H. Zhang, L. Tang, Z. Sun, G.-J. Zhang. Ultrasensitive Detection of MicroRNAs with Morpholino-Functionalized Nanochannel Biosensor. Analytical chemistry, 2017, 89, 5511.

[271] M. Ali, B. Yameen, R. Neumann, W. Ensinger, W. Knoll, O. Azzaroni. Biosensing and supramolecular bioconjugation in single conical polymer nanochannels. Facile incorporation of biorecognition elements into nanoconfined geometries. J. Am. Chem. Soc., 2008, 130, 16351.

[272] M. Ali, P. Ramirez, M.N. Tahir, S. Mafe, Z. Siwy, R. Neumann, W. Tremel, W. Ensinger. Biomolecular conjugation inside synthetic polymer nanopores via glycoproteinlectin interactions. Nanoscale, 2011, 3, 1894.

[273] S. Ahlawat, A. Nehra, V. Pandey, K.P. Singh. Gold-coated nanoporous polycarbonate track-etched solid platform for the rapid detection of mesothelin. Ionics, 2019, 25, 1887.

[274] N. Liu, Y. Jiang, Y. Zhou, F. Xia, W. Guo, L. Jiang. Two-way nanopore sensing of sequence-specific oligonucleotides and small-molecule targets in complex matrices using integrated DNA supersandwich structures. Angewandte Chemie (International ed. in English), 2013, 52, 2007. 


\section{WILEY-VCH}

[275] X. Xu, W. Zhao, P. Gao, H. Li, G. Feng, Z. Zhao, X. Lou. Coordination of the electrical and optical signals revealing nanochannels with an 'onion-like' gating mechanism and its sensing application. NPG Asia Mater, 2016, 8, e234-e234.

[276] W. Guo, F. Hong, N. Liu, J. Huang, B. Wang, R. Duan, X. Lou, F. Xia. Targetspecific 3D DNA gatekeepers for biomimetic nanopores. Advanced materials (Deerfield Beach, Fla.), 2015, 27, 2090.

[277] M. Ali, M.N. Tahir, Z. Siwy, R. Neumann, W. Tremel, W. Ensinger. Hydrogen peroxide sensing with horseradish peroxidase-modified polymer single conical nanochannels. Analytical chemistry, 2011, 83, 1673.

[278] G. Pérez-Mitta, A.S. Peinetti, M.L. Cortez, M.E. Toimil-Molares, C. Trautmann, O. Azzaroni. Highly Sensitive Biosensing with Solid-State Nanopores Displaying Enzymatically Reconfigurable Rectification Properties. Nano Lett., 2018, 18, 3303.

[279] A. Achilli, T.Y. Cath, A.E. Childress. Power generation with pressure retarded osmosis: An experimental and theoretical investigation. Journal of Membrane Science, 2009, $343,42$.

[280] A. Achilli, A.E. Childress. Pressure retarded osmosis: From the vision of Sidney Loeb to the first prototype installation - Review. Desalination, 2010, 261, 205.

[281] M. Turek, B. Bandura. Renewable energy by reverse electrodialysis. Desalination, 2007, 205, 67.

[282] A. Siria, P. Poncharal, A.-L. Biance, R. Fulcrand, X. Blase, S.T. Purcell, L. Bocquet. Giant osmotic energy conversion measured in a single transmembrane boron nitride nanotube. Nature, 2013, 494, 455.

[283] J. Feng, M. Graf, K. Liu, D. Ovchinnikov, D. Dumcenco, M. Heiranian, V. Nandigana, N.R. Aluru, A. Kis, A. Radenovic. Single-layer MoS2 nanopores as nanopower generators. Nature, 2016, 536, 197.

[284] Y. Feng, W. Zhu, W. Guo, L. Jiang. Bioinspired Energy Conversion in Nanofluidics: A Paradigm of Material Evolution. Advanced materials (Deerfield Beach, Fla.), 2017, 29. [285] T. Ma, E. Balanzat, J.-M. Janot, S. Balme. Nanopore Functionalized by Highly Charged Hydrogels for Osmotic Energy Harvesting. ACS applied materials \& interfaces, 2019, 11, 12578.

[286] T.B.H. Schroeder, A. Guha, A. Lamoureux, G. VanRenterghem, D. Sept, M. Shtein, J. Yang, M. Mayer. An electric-eel-inspired soft power source from stacked hydrogels. Nature, 2017, 552, 214.

[287] J.-P. Hsu, T.-C. Su, P.-H. Peng, S.-C. Hsu, M.-J. Zheng, L.-H. Yeh. Unraveling the Anomalous Surface-Charge-Dependent Osmotic Power Using a Single Funnel-Shaped Nanochannel. ACS nano, 2019, 13, 13374.

[288] G. Laucirica, A.G. Albesa, M.E. Toimil-Molares, C. Trautmann, W.A. Marmisollé, O. Azzaroni. Shape matters: Enhanced osmotic energy harvesting in bullet-shaped nanochannels. Nano Energy, 2020, 71, 104612.

[289] F. Xiao, D. Ji, H. Li, J. Tang, Y. Feng, L. Ding, L. Cao, N. Li, L. Jiang, W. Guo. A general strategy to simulate osmotic energy conversion in multi-pore nanofluidic systems. Mater. Chem. Front., 2018, 2, 935.

[290] J. Gao, X. Liu, Y. Jiang, L. Ding, L. Jiang, W. Guo. Understanding the Giant Gap between Single-Pore- and Membrane-Based Nanofluidic Osmotic Power Generators. Small (Weinheim an der Bergstrasse, Germany), 2019, 15, e1804279.

[291] Z. Zhang, X.-Y. Kong, K. Xiao, Q. Liu, G. Xie, P. Li, J. Ma, Y. Tian, L. Wen, L. Jiang. Engineered Asymmetric Heterogeneous Membrane: A Concentration-Gradient-Driven Energy Harvesting Device. J. Am. Chem. Soc., 2015, 137, 14765.

[292] Z. Zhang, P. Li, X.-Y. Kong, G. Xie, Y. Qian, Z. Wang, Y. Tian, L. Wen, L. Jiang. Bioinspired Heterogeneous Ion Pump Membranes: Unidirectional Selective Pumping and 


\section{WILEY-VCH}

Controllable Gating Properties Stemming from Asymmetric Ionic Group Distribution. J. Am. Chem. Soc., 2018, 140, 1083.

[293] a) M.A. Green, S.P. Bremner. Energy conversion approaches and materials for highefficiency photovoltaics. Nature materials, 2016, 16, 23; b) M.A. Green. Commercial progress and challenges for photovoltaics. Nat Energy, 2016, 1, 28; c) C.E.L. Latunussa, F. Ardente, G.A. Blengini, L. Mancini. Life Cycle Assessment of an innovative recycling process for crystalline silicon photovoltaic panels. Solar Energy Materials and Solar Cells, 2016, 156, 101;

[294] a) P. Cheng, G. Li, X. Zhan, Y. Yang. Next-generation organic photovoltaics based on non-fullerene acceptors. Nature Photon, 2018, 12, 131; b) G.E. Eperon, T. Leijtens, K.A. Bush, R. Prasanna, T. Green, J.T.-W. Wang, D.P. McMeekin, G. Volonakis, R.L. Milot, R. May, A. Palmstrom, D.J. Slotcavage, R.A. Belisle, J.B. Patel, E.S. Parrott, R.J. Sutton, W. Ma, F. Moghadam, B. Conings, A. Babayigit, H.-G. Boyen, S. Bent, F. Giustino, L.M. Herz, M.B. Johnston, M.D. McGehee, H.J. Snaith. Perovskite-perovskite tandem photovoltaics with optimized band gaps. Science (New York, N.Y.), 2016, 354, 861; c) F. Priolo, T.

Gregorkiewicz, M. Galli, T.F. Krauss. Silicon nanostructures for photonics and photovoltaics. Nature nanotechnology, 2014, 9, 19;

[295] L. Wen, X. Hou, Y. Tian, J. Zhai, L. Jiang. Bio-inspired Photoelectric Conversion Based on Smart-Gating Nanochannels. Adv. Funct. Mater., 2010, 20, 2636.

[296] Z. Meng, H. Bao, J. Wang, C. Jiang, M. Zhang, J. Zhai, L. Jiang. Artificial ion channels regulating light-induced ionic currents in photoelectrical conversion systems. Advanced materials (Deerfield Beach, Fla.), 2014, 26, 2329.

[297] X. Xie, G.A. Crespo, G. Mistlberger, E. Bakker. Photocurrent generation based on a light-driven proton pump in an artificial liquid membrane. Nature chemistry, 2014, 6, 202. [298] a) A. Majumdar. Materials science. Thermoelectricity in semiconductor nanostructures. Science (New York, N.Y.), 2004, 303, 777; b) P. Reddy, S.-Y. Jang, R.A. Segalman, A. Majumdar. Thermoelectricity in molecular junctions. Science (New York, N.Y.), 2007, 315, 1568; c) G. Marchegiani, A. Braggio, F. Giazotto. Nonlinear Thermoelectricity with ElectronHole Symmetric Systems. Physical review letters, 2020, 124, 106801;

[299] a) L. Fu, S. Merabia, L. Joly. What Controls Thermo-osmosis? Molecular Simulations Show the Critical Role of Interfacial Hydrodynamics. Physical review letters, 2017, 119,

214501 ; b) L. Fu, S. Merabia, L. Joly. Understanding Fast and Robust Thermo-osmotic Flows through Carbon Nanotube Membranes: Thermodynamics Meets Hydrodynamics. The journal of physical chemistry letters, 2018, 9, 2086; c) L. Fu, L. Joly, S. Merabia. Giant

Thermoelectric Response of Nanofluidic Systems Driven by Water Excess Enthalpy. Physical review letters, 2019, 123, 138001;

[300] G. Xie, P. Li, Z. Zhang, K. Xiao, X.-Y. Kong, L. Wen, L. Jiang. Skin-Inspired LowGrade Heat Energy Harvesting Using Directed Ionic Flow through Conical Nanochannels. Adv. Energy Mater., 2018, 8, 1800459.

[301] K. Chen, L. Yao, F. Yan, S. Liu, R. Yang, B. Su. Thermo-osmotic energy conversion and storage by nanochannels. J. Mater. Chem. A, 2019, 7, 25258. 


\section{WILEY-VCH}

(a)

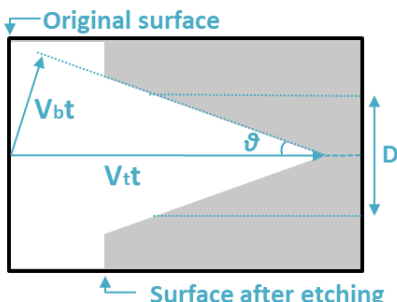

(c)

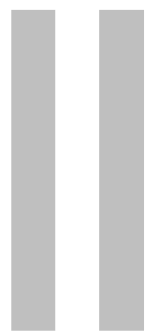

Cylinder

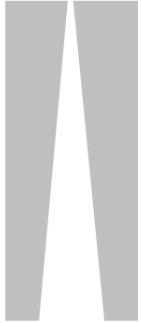

Cone (b)
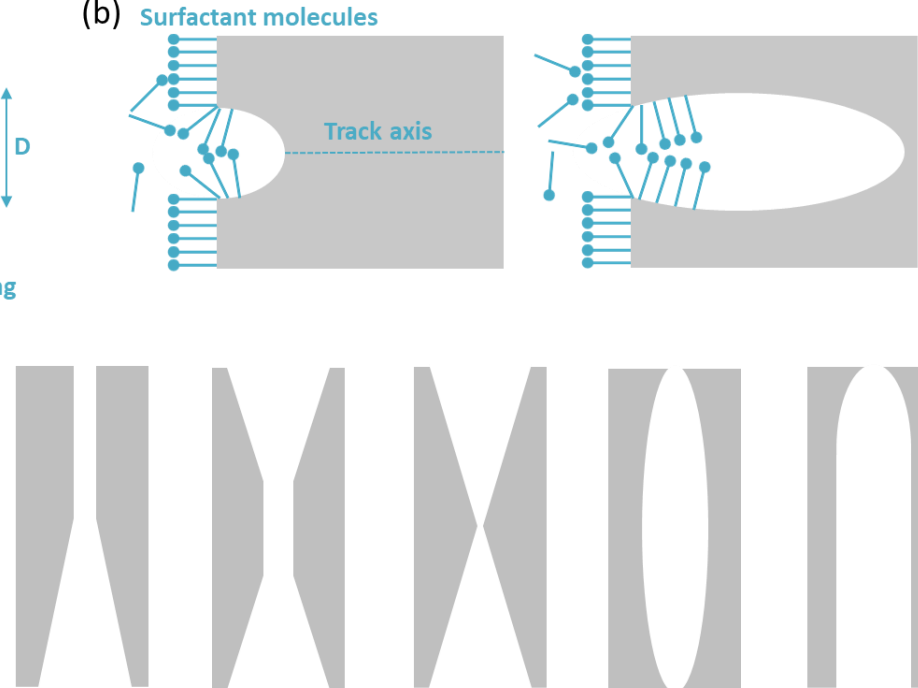

Funnel

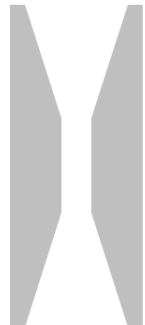

Dumbbell

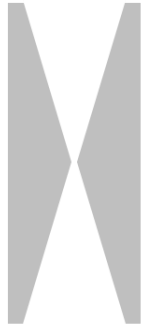

Hourglass

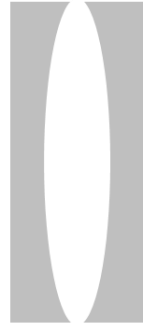

Cigar

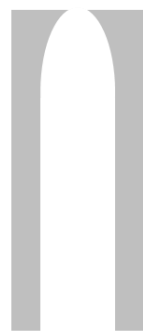

Bullet

Figure 1. (a) Schematic representation of chemical etching process on an across section. (b)

Schematic representation of a chemical etching process with participation of surfactant molecules. (c) Different geometries in track-etched nanopores. 
WILEY-VCH

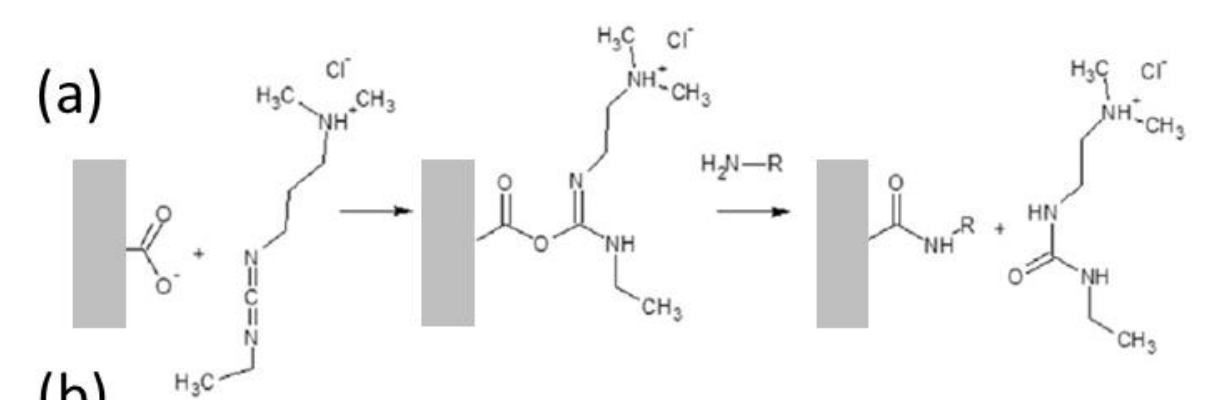

(b)

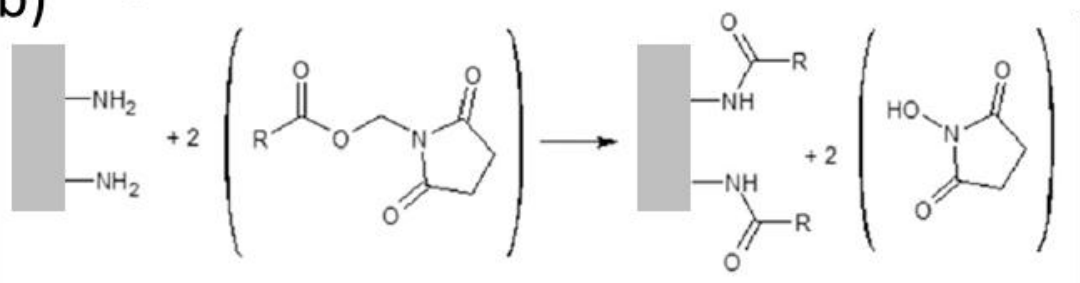

Figure 2. Schematic representation of carboxamide formations by (a) EDC and (b) NHS reproduced with permission [55], 2017, Elseiver. 


\section{WILEY-VCH}

(a)

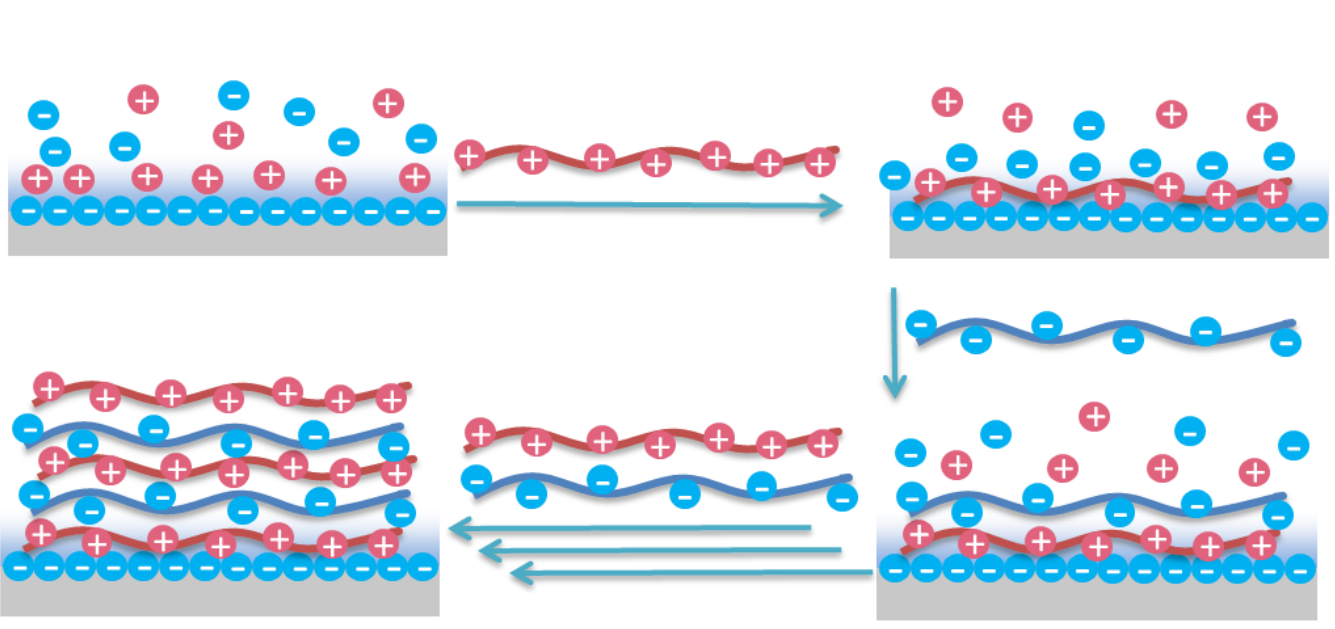

(b) i

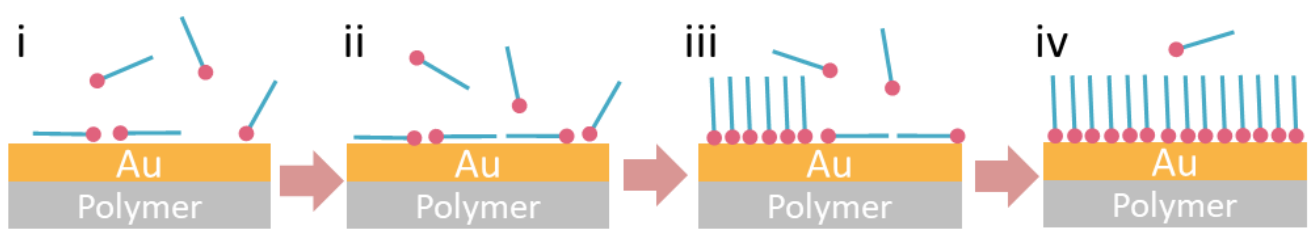

Figure 3. (a) Schematic presentation of layer-by-layer polyelectrolytes adsorption. (b)

Schematic representation of thiol SAMs formation at step (i) physisorption, step (ii) lying formation phase, step (iii) standing nucleation phase and step (iv) completion phase 


\section{WILEY-VCH}

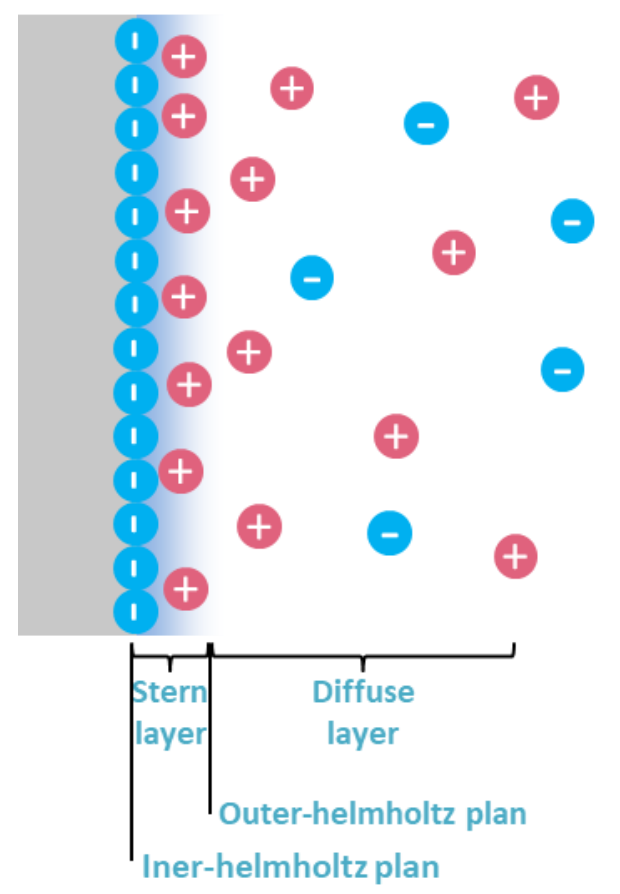

Figure 4 Scheme of electrical double layer. 


\section{WILEY-VCH}

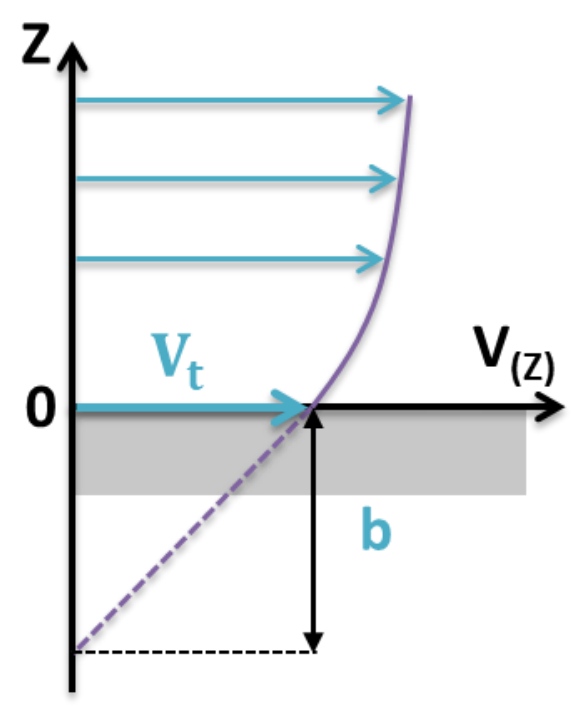

Figure 5 Sketch of liquid velocity profile close to surface and the slip length $b$. 


\section{WILEY-VCH}
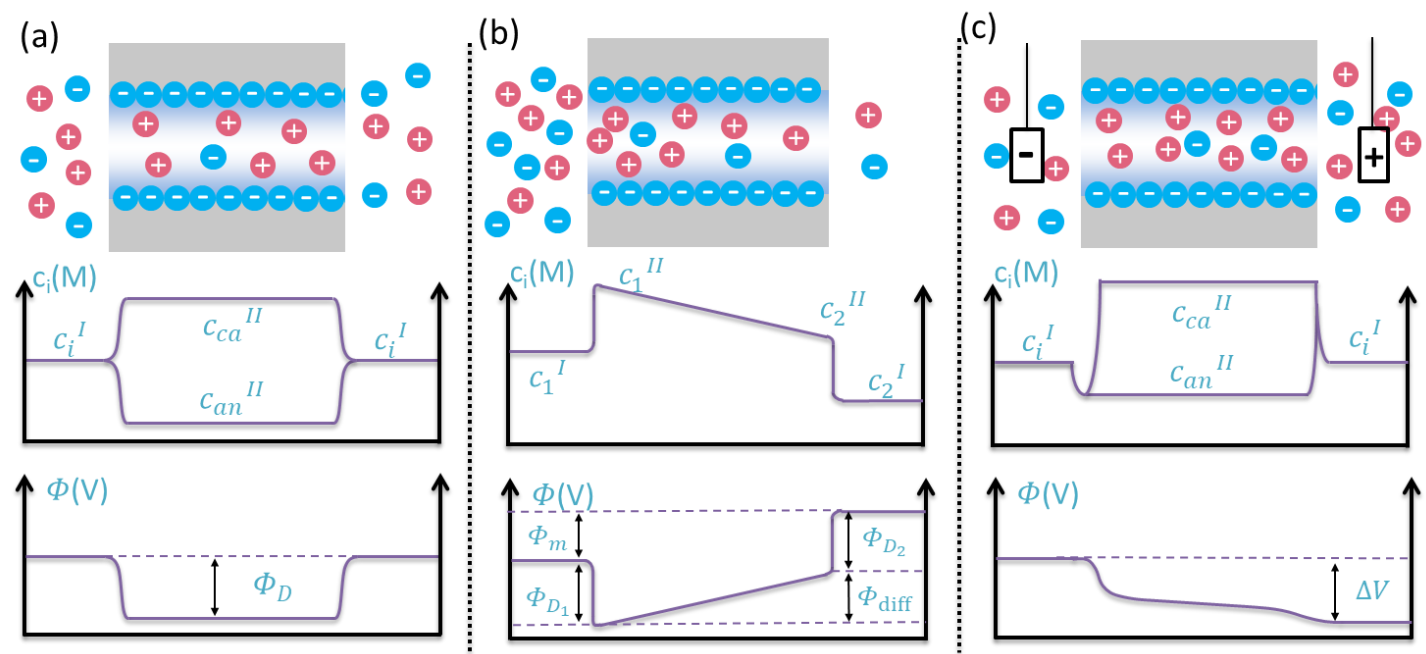

Figure 6 (a) Concentration and potential profiles along negatively charged membrane length separating two solutions with the same concentration. (b) Concentration and potential profiles along negatively charged membrane length separating two solutions with the same concentration under large applied electric bias. (c) Concentration and potential profiles along negatively charged membrane length separating two solutions with different concentrations. 


\section{WILEY-VCH}

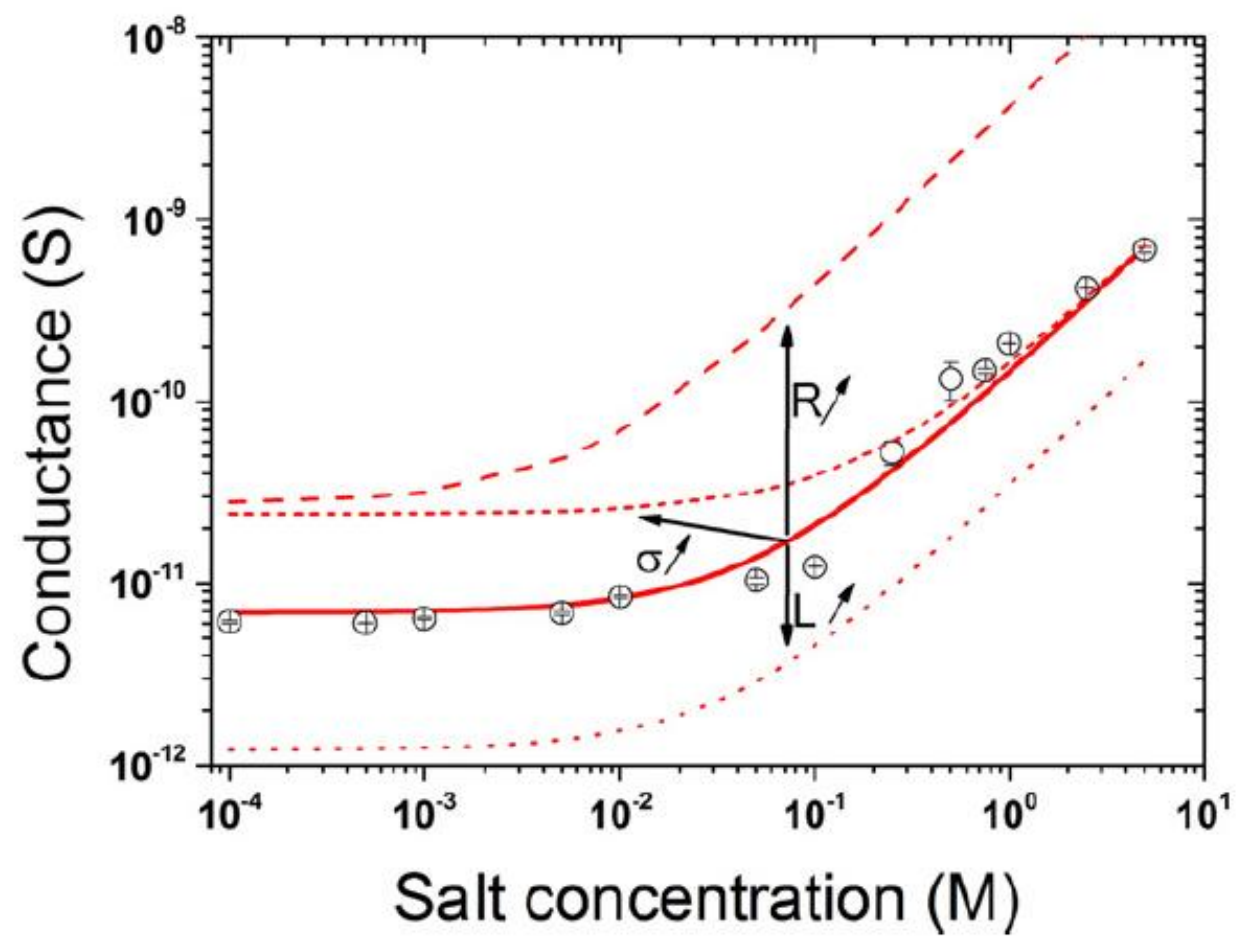

Figure 7 Schematic behavior of a nanopore conductance as a function of the electrolyte concentration 1:1 ( $\mathrm{NaCl}$ or $\mathrm{KCl}$ type). In full line is reported the fit using Eq. (30) and in dash lines are reported the impact of an increase of the surface charge density $\sigma$, the radius $\mathrm{R}$ or the length L of the nanopore on its conductance. Reproduced with permission [55], 2017, Elsevier. 


\section{WILEY-VCH}

(a)

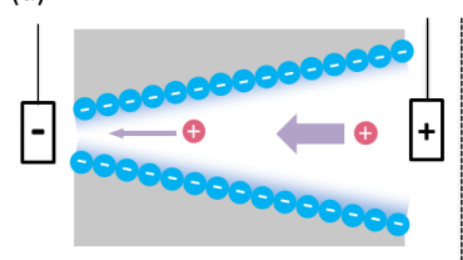

(b)

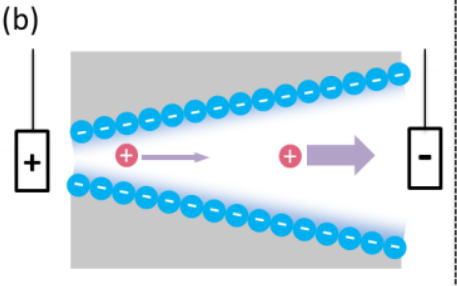

(c)

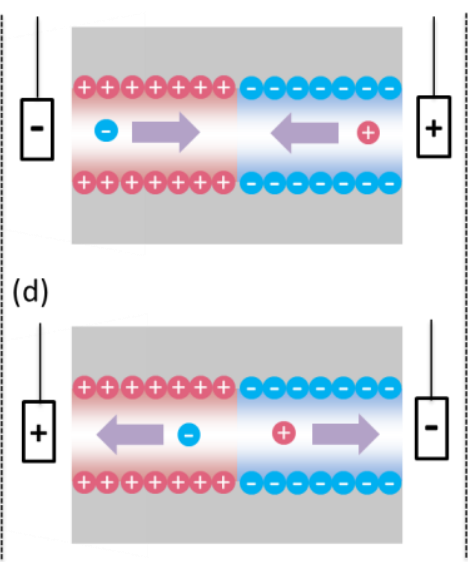

(e)

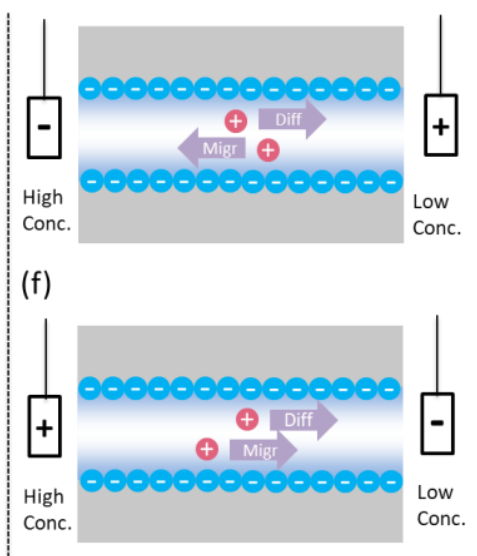

Figure 8 Ion current rectification behavior due to asymmetry from (a) and (b) shape , (c) and (d) charge distribution and (e) and (f) concentration gradient. For (a) (c) and (f), ions are accumulated in channel corresponding to an open state and for (b) (d) and (e), ions are depleted in channel corresponding to a closed state. 
(a)

(b) Poly(methacryoyl-L-Lysine)

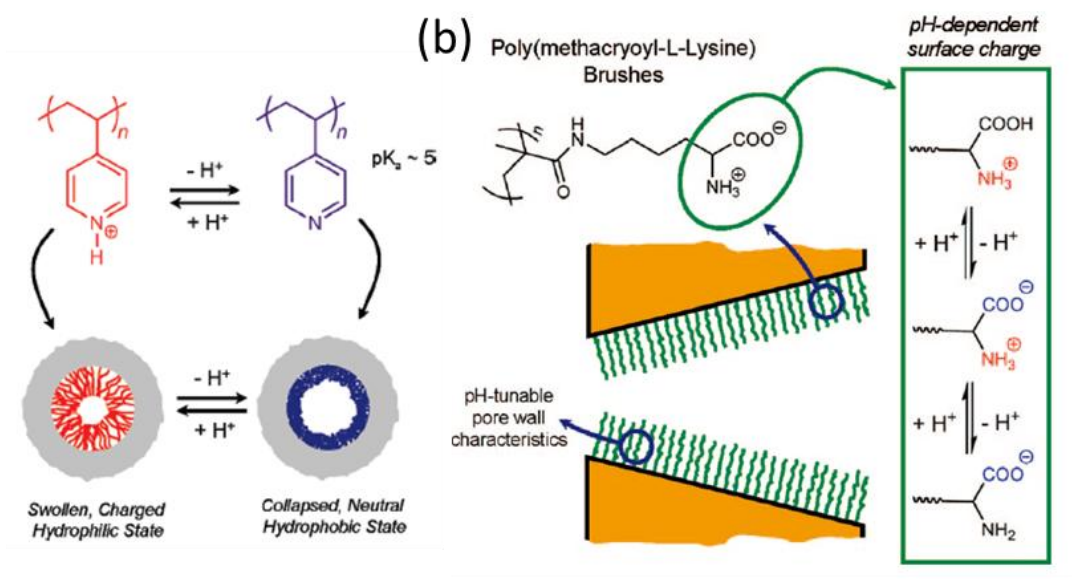

(c)

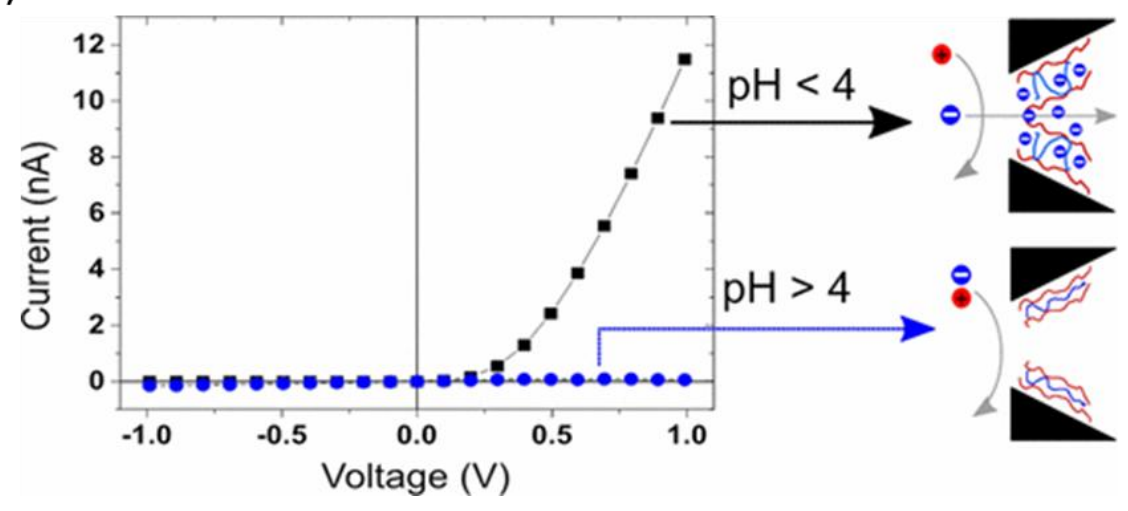

Figure 9 pH-responsive nanochannels. (a) P4VP functionalized nanochannel reproduced with permission [194], 2009, American Chemical Society, (b) PMALys functionalized nanochannel reproduced with permission [195], 2009, American Chemical Society, (c) PEI/ChS layer-by-layer functionalized nanochannel reproduced with permission [40], 2017, American Chemical Society. 


\section{WILEY-VCH}

(a) DNA with uniform charge $(\mathrm{pH} 8)$
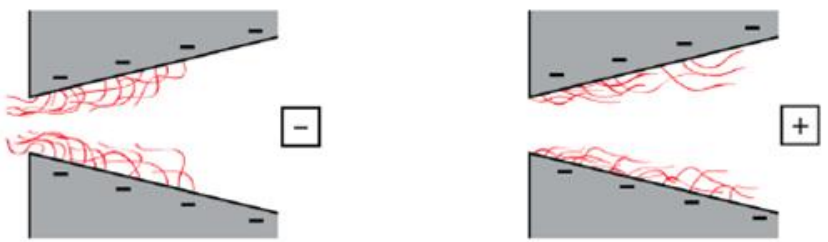

DNA with zwitterionic nucleotides $(\mathrm{pH} \mathrm{5.5)} \mathrm{-} \mathrm{Negative} \mathrm{charge}$

(b)

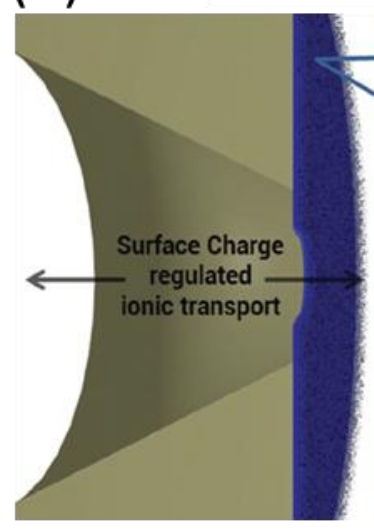

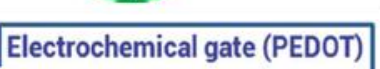

Electrochemical gate (PEDOT)
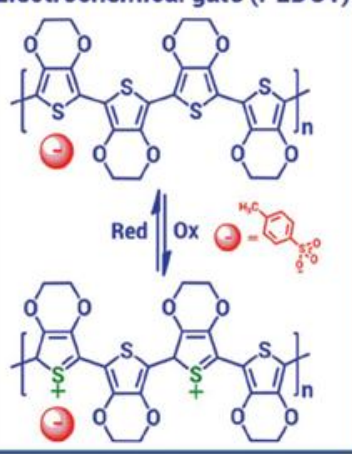

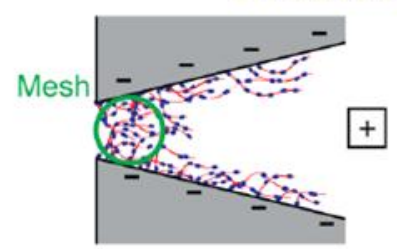

(c)
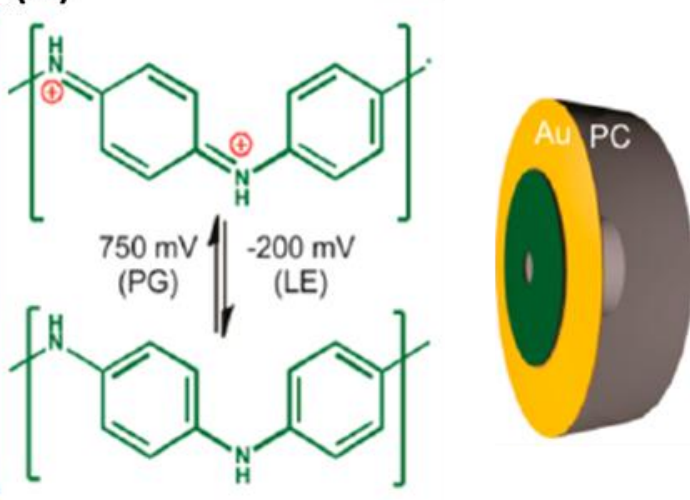

Figure 10 Potential responsive nanochannels functionalized by (a) DNA[199], (b) PEDOT reproduced with permission [201], 2017, Wiley and (c) PANI reproduced with permission [200], 2015, American Chemical Society. 


\section{WILEY-VCH}

(a)

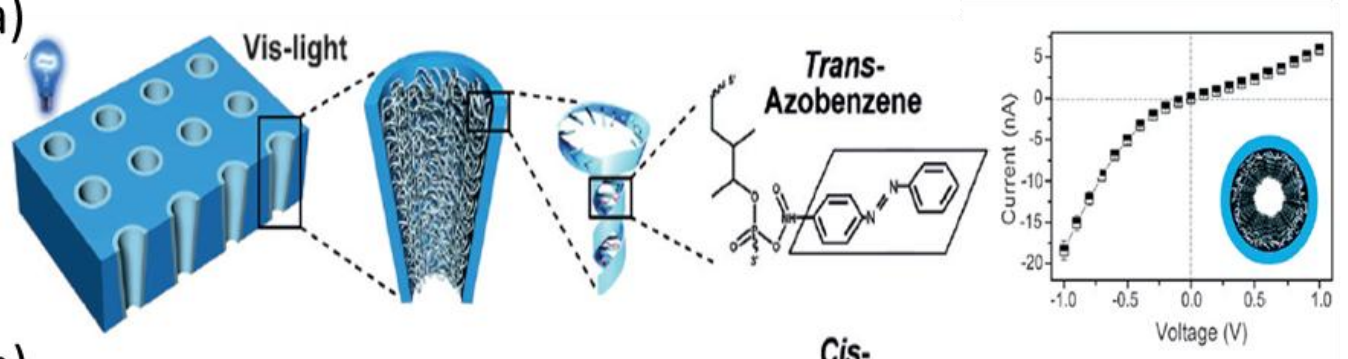

(b)
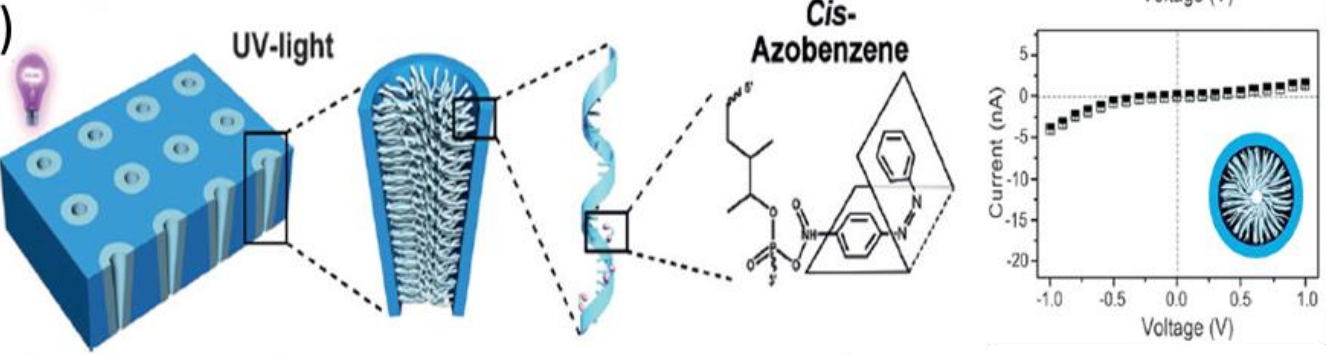

(c)
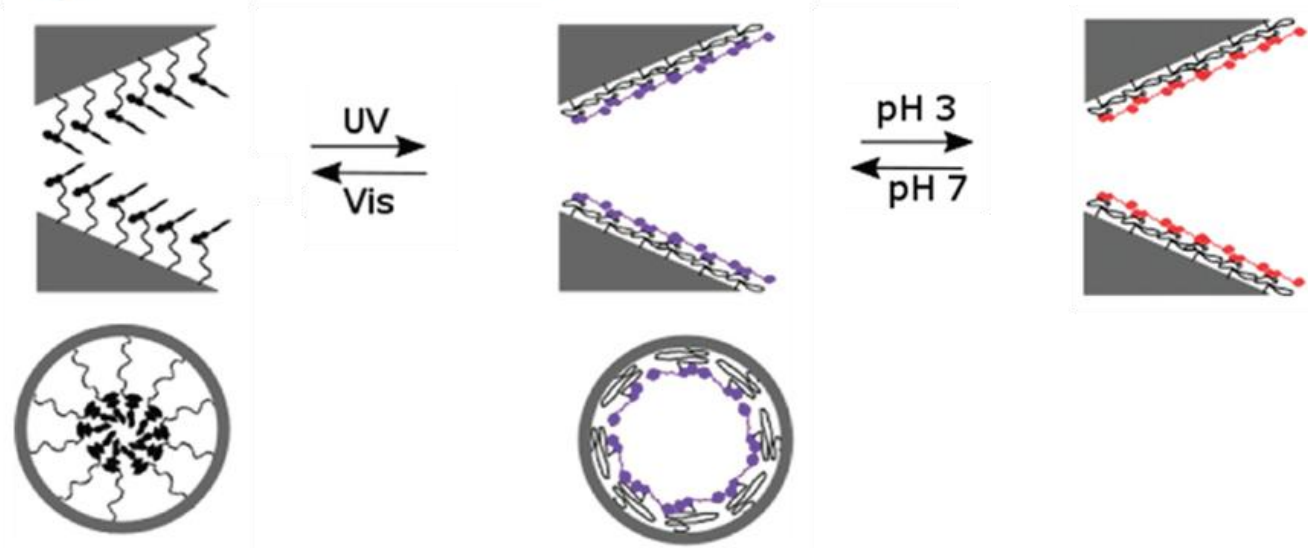

Figure 11 UV-Vis light-responsive nanochannel functionalized by azobenzene-DNA in (a)

Vis light and in (b) UV light reproduced with permission [207], 2016, Wiley. (c) SpiropyranPEG functionalized nanopore under UV/Vis light and solutions of $\mathrm{pH} 3$ and $\mathrm{pH} 7$ reproduced with permission [100], 2018, Wiley. 


\section{WILEY-VCH}

(a)

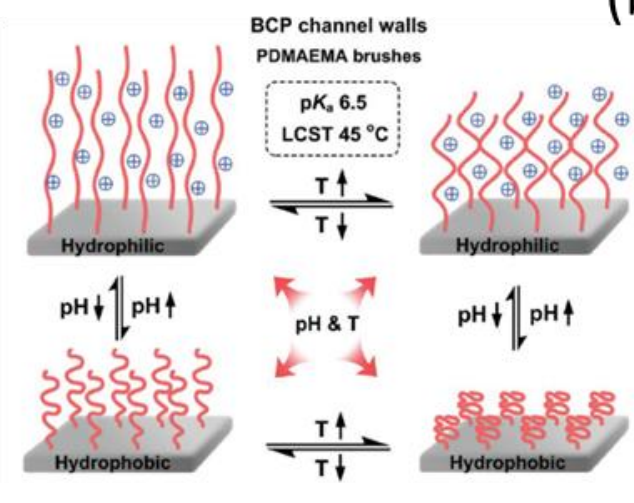

(b)

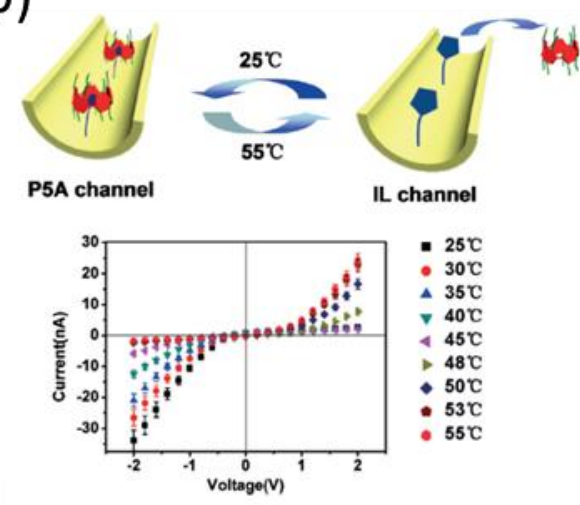

Figure 12 Thermal-responsive nanochannel functionalized by (a) PDMAEMA brushes reproduced with permission [213], 2016, Wiley and (b) P5A/IL host guest reproduced with permission [214], 2017, Wiley. 
WILEY-VCH

(a)

(b)

(c)
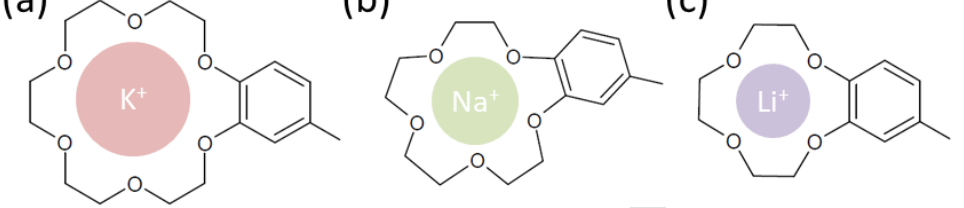

(d)

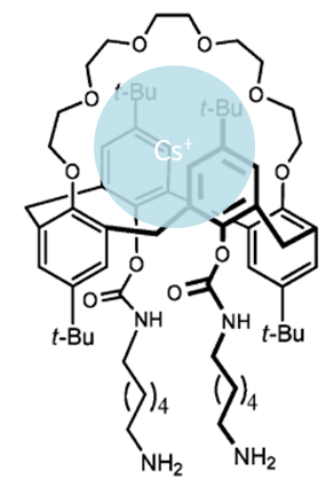

(e)

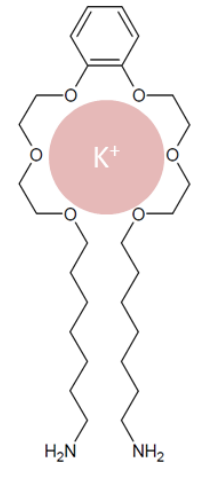

Figure 13 Crown ether cage molecules (a) B18C6, (b) B15C5, (c) B12C4, (d) t-BuC[4]C, (e) bis-pod and their corresponding alkali metal ions. 


\section{WILEY-VCH}

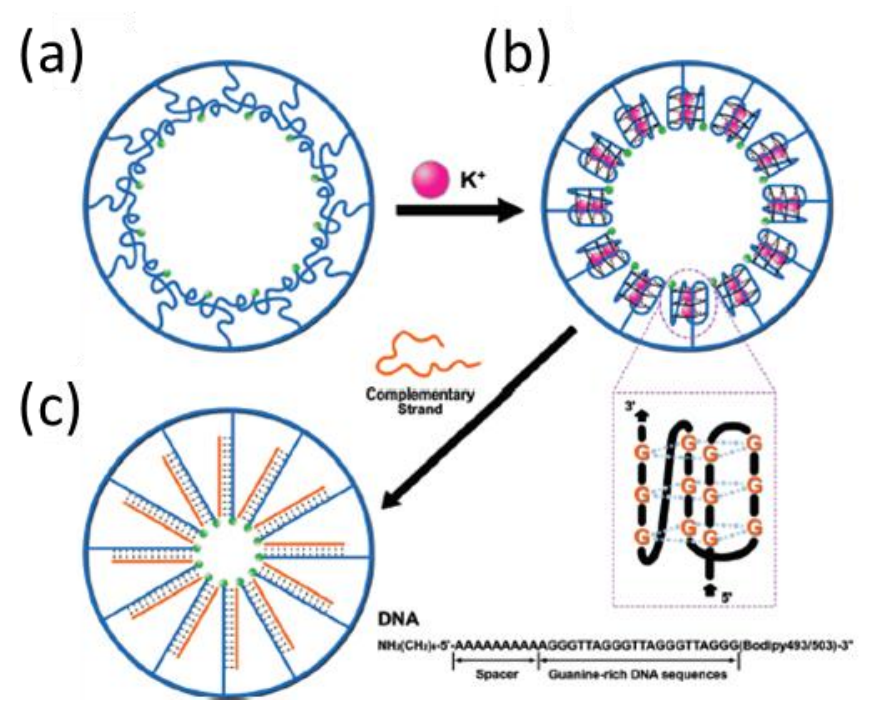

Figure 14 G4 DNA functionalized $\mathrm{K}^{+}$sensors. (a) Loosely packed single-stranded DNA without $\mathrm{K}^{+}$. (b) Densely packed rigid quadruplex structures with $\mathrm{K}^{+}$. (c) $\mathrm{K}^{+}$departure with participation of the complementary strand reproduced with permission [219], 2009, American Chemical Society. 


\section{WILEY-VCH}

(a)

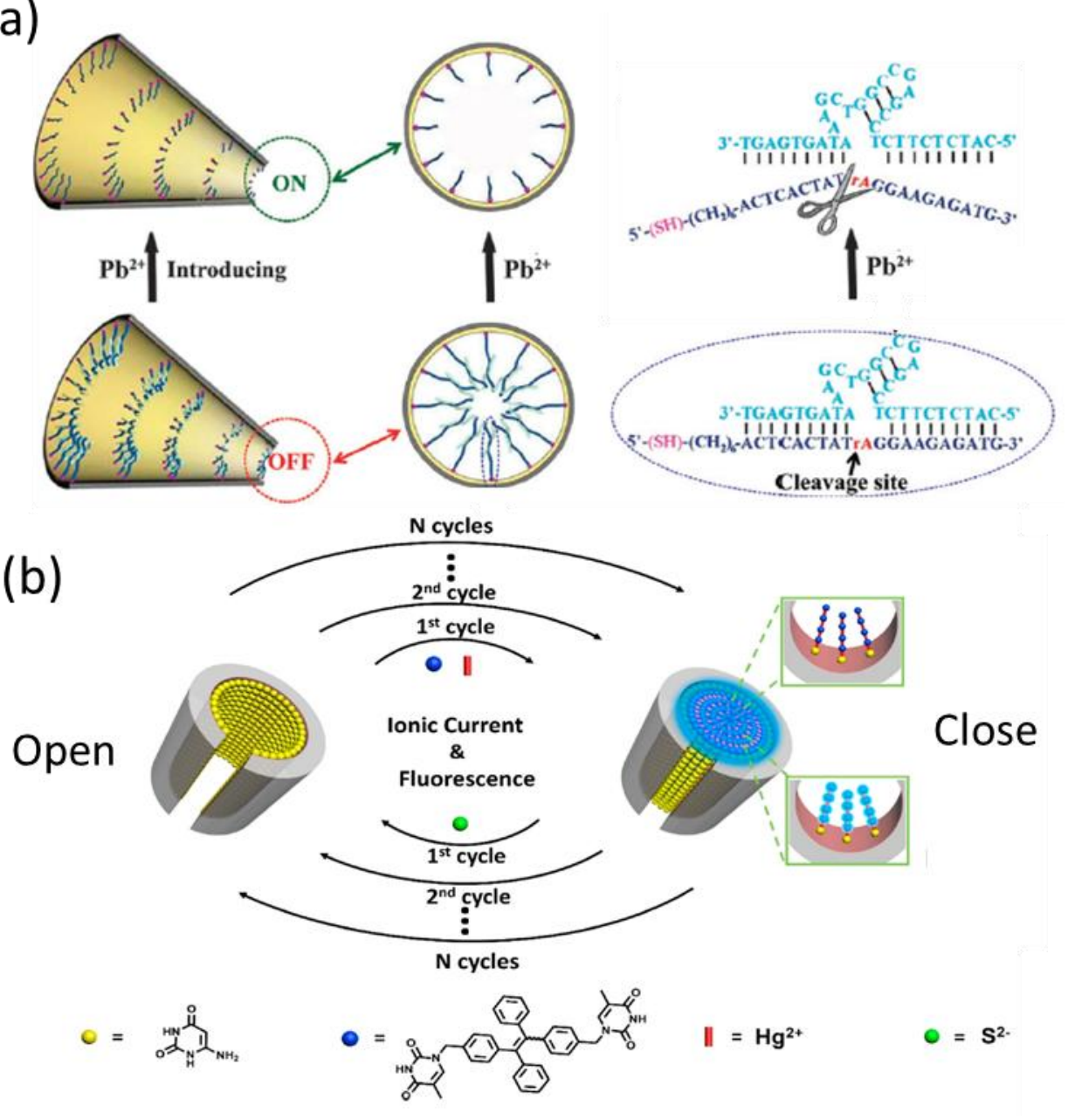

Figure 15 Ions sensing by macro structure construction/destruction. (a) DNAzyme functionalized nanochannel between on and off state with $\mathrm{Pb}^{2+}$ due to its cleavage effect reproduced with permission [222], 2015, Royal Society of Chemistry. (b) TPE-2D/Hg ${ }^{2+}$ formed macro structure and its destruction by $\mathrm{S}^{2-}$ as $\mathrm{Hg}^{2+} / \mathrm{S}^{2-}$ sensor reproduced with permission [223], 2016, American Chemical Society. 
WILEY-VCH

(a)

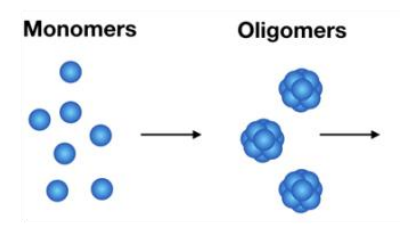

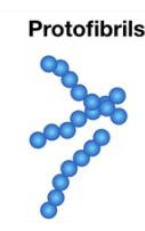

(b)

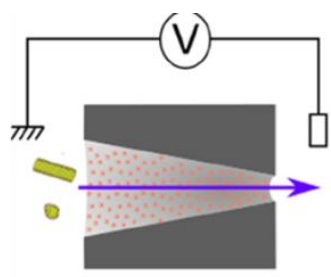

(c)
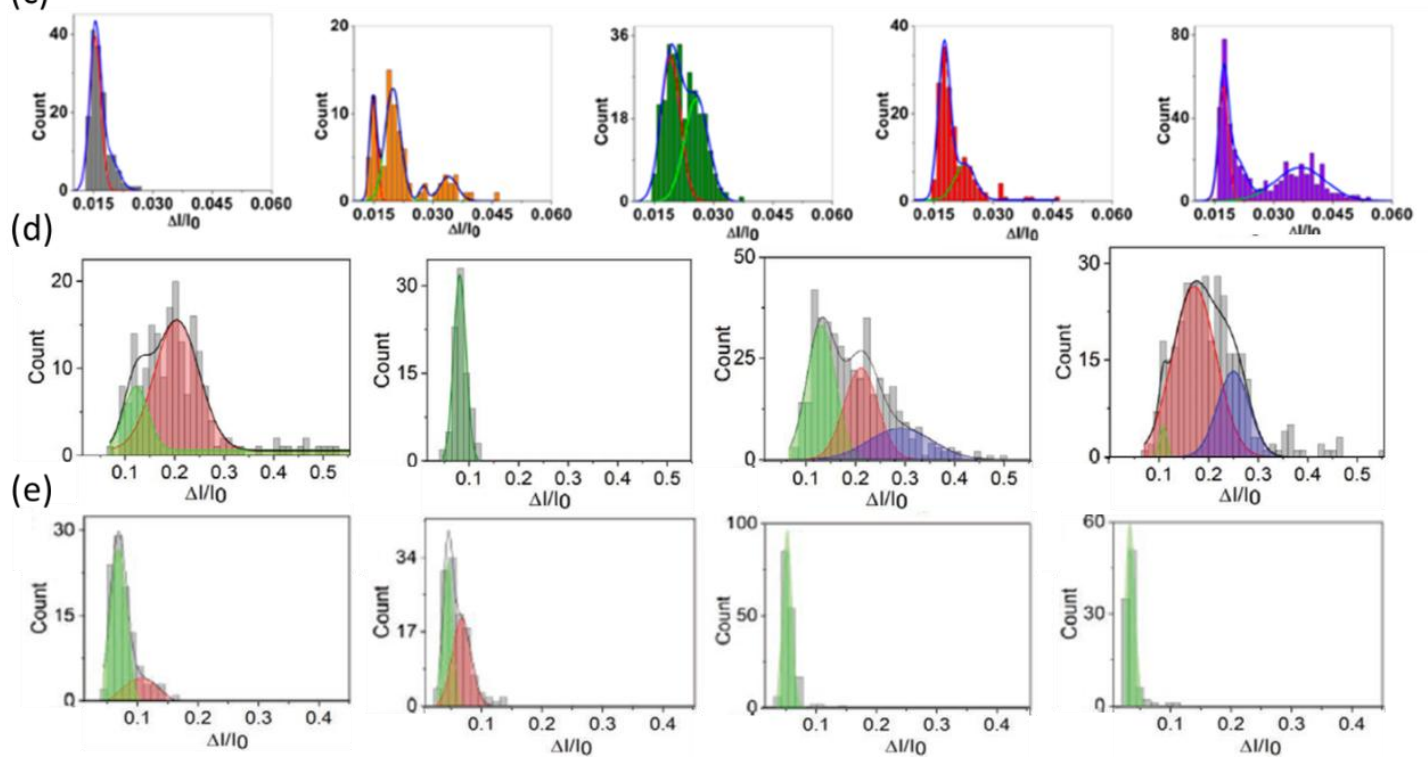

Figure 16 (a) Schematic representation of the amyloid aggregation pathway. (b) Schematic representation of the amyloid translocation in conical nanopore reproduced with permission [258], 2018, American Chemical Society. (c) The histogram of relative current blockade for $\beta$-lactoglobulin amyloids at $3 \mathrm{~h}, 6 \mathrm{~h}, 16 \mathrm{~h}, 24 \mathrm{~h}, 34 \mathrm{~h}$ measured in a nanopore of $\mathrm{D}_{\text {tip }}=7.6 \mathrm{~nm}$, $D_{\text {base }}=600 \mathrm{~nm}, \mathrm{~L}=6 \mu \mathrm{m}$ reproduced with permission [258], 2018, American Chemical Society. (d) The histograms of relative current blockade of enzymatic degradation of $\beta$-lactoglobulin $30 \mathrm{~h}$ amyloids by pepsin during $0 \mathrm{~min}, 24 \mathrm{~min}, 137 \mathrm{~min}, 350 \mathrm{~min}, 2018$, reproduced with permission [260], 2020, Wiley. (e) The histograms of relative current blockade of enzymatic degradation of $\beta$-lactoglobulin $30 \mathrm{~h}$ amyloids by trypsin during $0 \mathrm{~min}, 46 \mathrm{~min}, 129 \mathrm{~min}, 303$ min, reproduced with permission [260], 2020, Wiley. 


\section{WILEY-VCH}

(a)

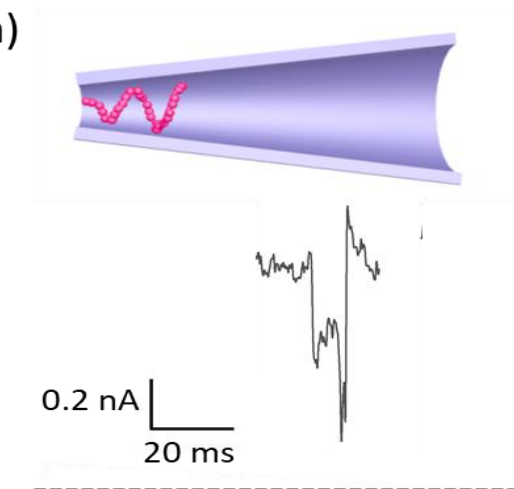

(c)

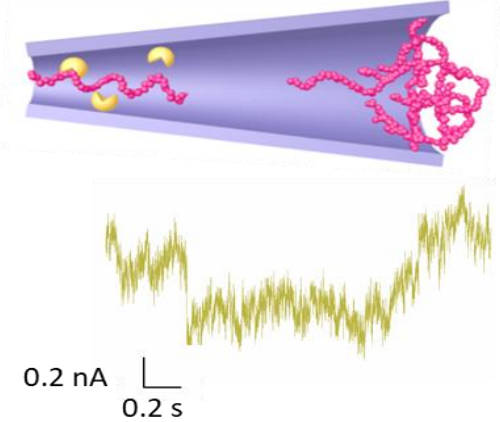

(b)

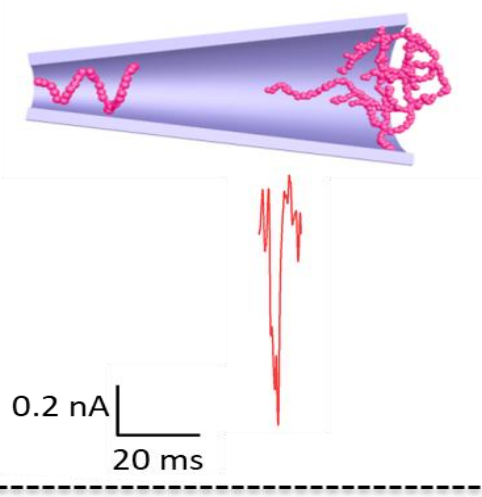

(d)

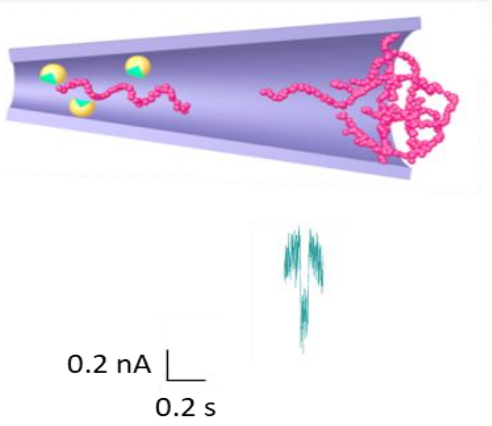

Figure 17 Schematic representations and signal zoom of hyaluronic acid translocation through conical nanopore in dilute regime (a), in the semi-dilute regime (b), in the semi-dilute regime with enzymes (c), in the semi-dilute regime with inhibited enzymes (d) reproduced with permission [139], 2020, Royal Society of Chemistry. 


\section{WILEY-VCH}

(a)

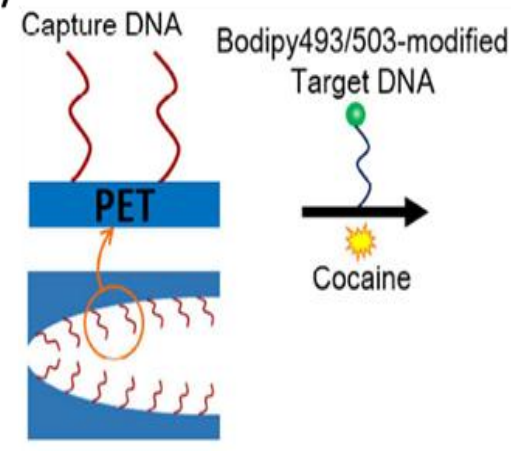

(b)

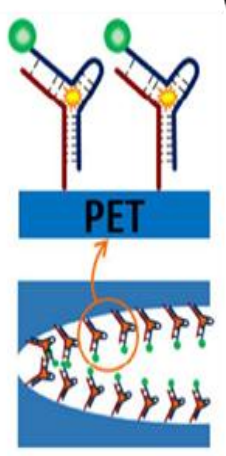

(c)

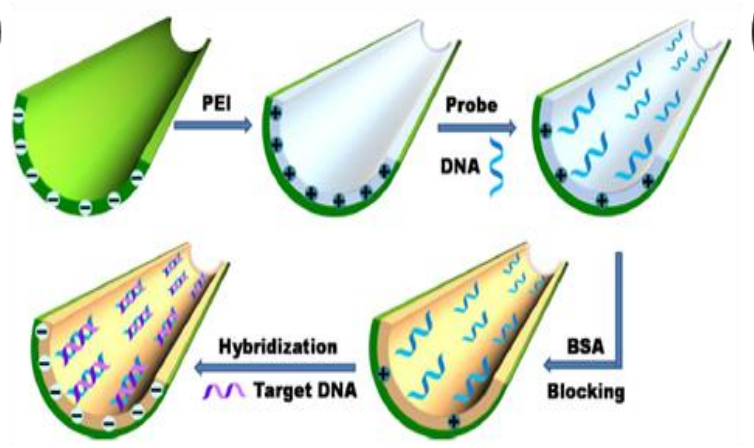

(e)

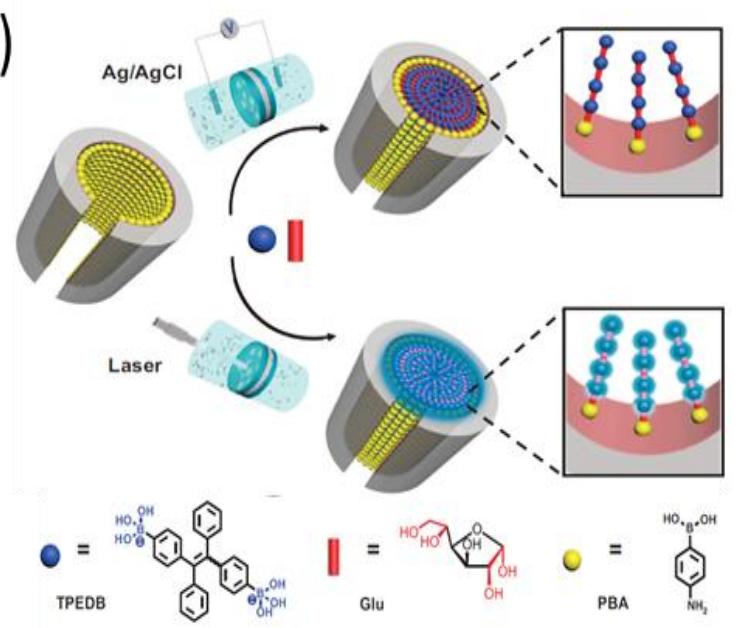

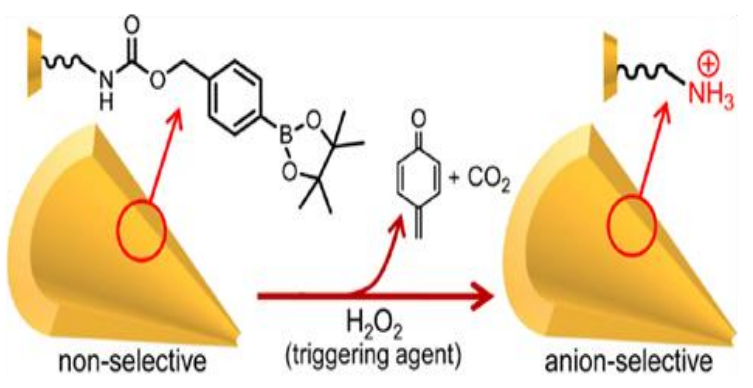

(d)

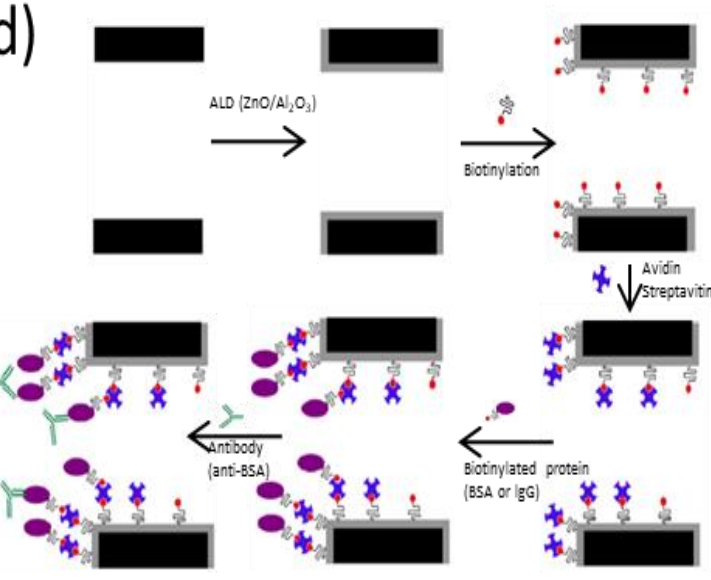

(f)

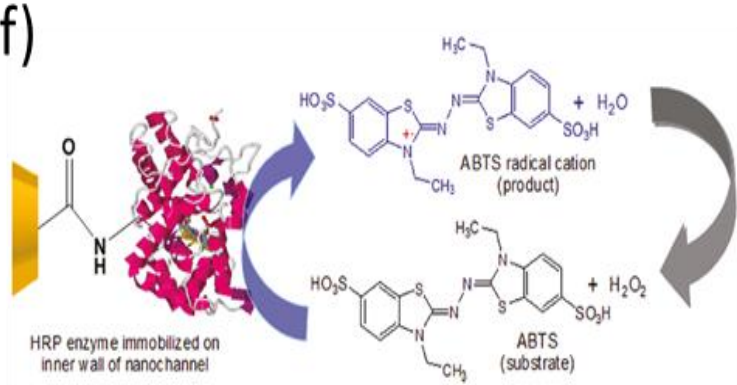

Figure 18 Molecule sensing by (a) trapping small molecule : cocaine molecules trapped in DNA probe reproduced with permission [264] 2018, American Chemical Society, (b) molecule-functional group reactions: $\mathrm{H}_{2} \mathrm{O}_{2}$ oxidation of $\mathrm{BEC}$ reproduced with permission [266] 2015, American Chemical Society, (c) nucleic acid hybridations: DNA probe and target DNA hybridization reproduced with permission [269], 2016, Elsevier (d) biomolecular recognition: recognition of biotin/avidin and protein/antibodies reproduced with permission [85] 2016, Elsevier, (e) macro structure construction/destruction: TPEDB and Glu for 3D constructions reproduced with permission [275], 2016, Nature Publishing Group, (f) enzyme- 


\section{WILEY-VCH}

substrate reactions: HRP enzyme with ABTS reproduced with permission [277], 2011, American Chemical Society.

(a)

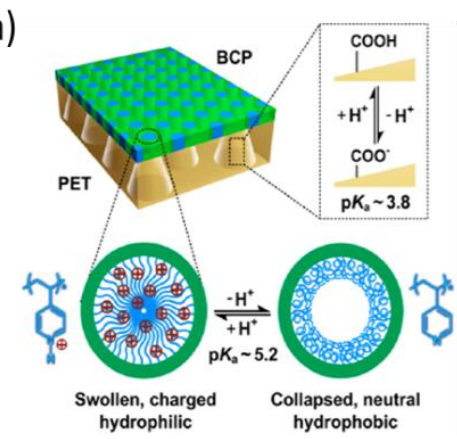

(b)

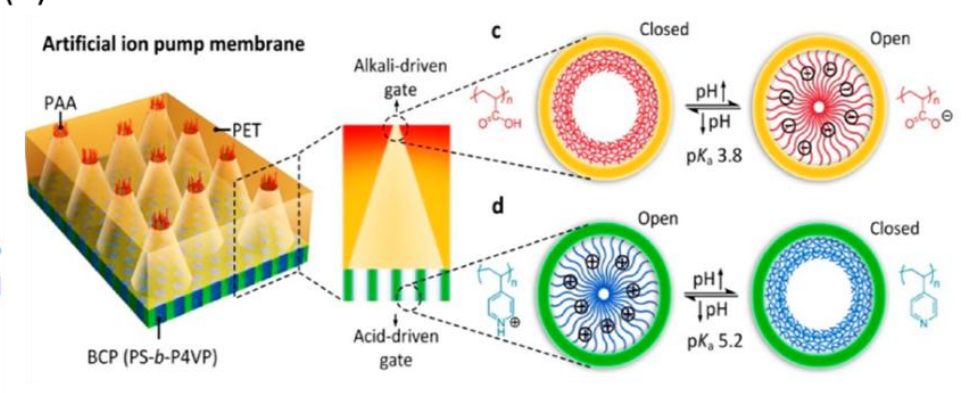

Figure $19 \mathrm{pH}$ controlled high ion selective nanochannels functionalized by (a) $\mathrm{PS}_{48400}$-b$\mathrm{P}_{4} \mathrm{VP}_{21300}$ coating reproduced with permission [291], 2015, American Chemical Society and (b) PS-b-P4VP/PAA coating reproduced with permission [292], 2018, American Chemical Society. 


\section{WILEY-VCH}

(a)

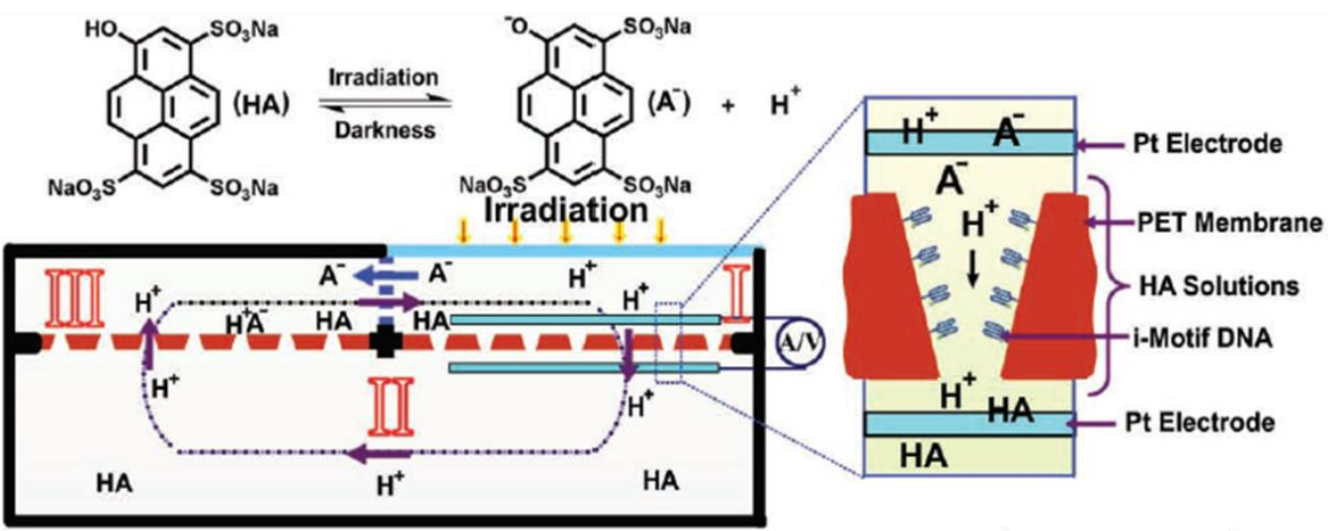

(b)

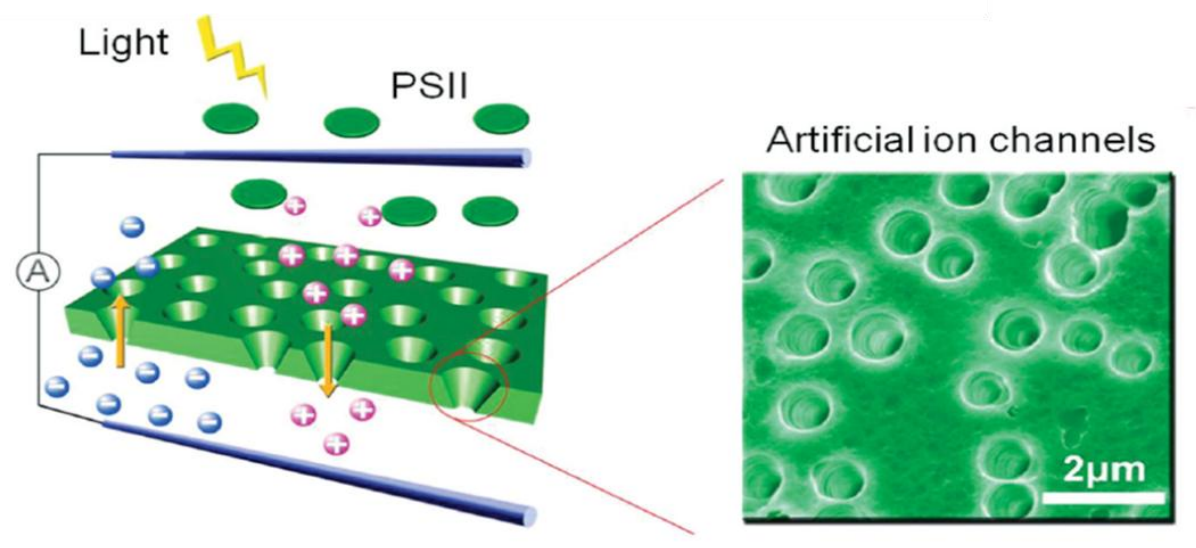

Figure 20 (a) Scheme of the photoelectric conversion system, which is constructed using a photoelectrochemical cell containing three parts, whereby only part I could be irradiated by outside light. An anion-exchange membrane (blue) is placed between part I and part III; and two pieces of membranes with proton-driven nanochannels were placed between part I and part II, and between part II and part III. 8-hydroxypyrene-1,3,6-trisulfonate (HA) was utilized as light-driven proton pumps. The generated protons could transport across the membrane between part I and part II and resulted in a charge imbalance on both sides of the membrane, thus, creating an $E$ dif . The total proton-transfer process is illustrated by the dashed rectangular curve in the scheme. Furthermore, the anions A - in part III can accept protons and recombine to the original HA molecules so as to supplement the HA molecules in part I. The output photocurrent relates to the redox reactions on the platinum electrodes, reproduced with permission [295], 2010, Wiley. (b) Scheme of Artificial ion channel in the photoelectric conversion system (PECS; bottom). The function of the PET multipore membranes in the 


\section{WILEY-VCH}

PECS is regulating the photocurrent by regulating ion transport, the same with that in the natural counterpart reproduced with permission [296], 2014, Nature Publishing Group. 


\section{WILEY-VCH}

This review gives an overview on the track-etched nanopore/membrane from their design to their applications. The different steps of fabrication and the strategy of functionalization are discussed. Then, the ionic transport properties under confined space are reminded. Then, the application of the track-etched nanopore/membrane to Stimuli-responsive ion channels, Ion gate, molecule sensing and energy production were shown and discussed.

Keyword: nanopore, track-etched, membrane, sensor, osmotic energy, biomimetic

Tianji Ma, Jean-Marc Janot, Sébastien Balme *

\section{Track-etched Nanopore/Membrane: From Fundamental to Applications}

ToC figure ((Please choose one size: $55 \mathrm{~mm}$ broad $\times 50 \mathrm{~mm}$ high or $110 \mathrm{~mm}$ broad $\times 20 \mathrm{~mm}$ high. Please do not use any other dimensions))

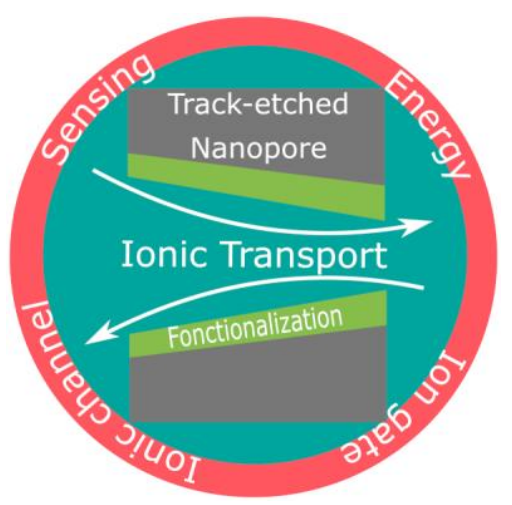

